\title{
Superstrings in Higher Order Extensions of Finsler Superspaces
}

\author{
Sergiu I. Vacaru \\ Institute of Applied Physics, Academy of Sciences, \\ 5 Academy str., Chişinău 2028, Republic of Moldova
}

Fax: 011-3732-738149, E-mail: lises@cc.acad.md

\begin{abstract}
The work proposes a general background of the theory of field interactions and strings in spaces with higher order anisotropy. Our approach proceeds by developing the concept of higher order anisotropic superspace which unifies the logical and mathematical aspects of modern KaluzaKlein theories and generalized Lagrange and Finsler geometry and leads to modelling of physical processes on higher order fiber bundles provided with nonlinear and distingushed connections and metric structures. The view adopted here is that a general field theory should incorporate all possible anisotropic and stochastic manifestations of classical and quantum interactions and, in consequence, a corresponding modification of basic principles and mathematical methods in formulation of physical theories.

The presentation is divided into two parts. The first five sections cover the higher order anisotropic superspaces. We focus on the geometry of distinguished by nonlinear connection vector superbundles, consider different supersymmetric extensions of Finsler and Lagrange spaces and analyze the structure of basic geometric objects on such superspaces. The remaining five sections are devoted to the theory of higher order anisotropic superstrings. In the framework of supersymmetric nonlinear sigma models in Finser extended backgrounds we prove that the lowenergy dynamics of such strings contains motion equations for locally anisotropic field interactions.

Our work is to be compared with important previous variants of extension of Finsler geometry and gravity (see, for instance, [7], 76, 74, 14]). There are substantial differences, because we rely on modeling of higher order anisotropic interactions on superbundle spaces and do not propose
\end{abstract}


some "exotic" Finsler models but a general approach which for trivial or corresponding parametization of nonlinear connection stuctures reduces to Kaluza-Klein and another variants of compactified higher-dimension space-times. The geometry of nonlinear connections (not being confused with connections for nonlinear realizations of gauge supergroups) is firstly considered for superspaces and possible cosequences on nonlinear connection field for compatible propagations of strings in anisotropic backgrounds are analyzed.

Finally, we note that the developed computation methods are general (in some line very similar to those for Einstein-Cartan-Weyl spaces which is a priority comparing with another combersome calculations in Finsler geometry) and admit extension to various Clifford and spinor bundles.

(c) Sergiu I. Vacaru, 1996

\section{Contents}

\begin{tabular}{lll}
\hline 1 & Introduction & 3
\end{tabular}

\begin{tabular}{|lll}
2 & Distinguished Superbundles & 5
\end{tabular}

$2.1 \quad$ Supermanifolds and superbundles . . . . . . . . . . . . . . . . . 5

2.2 Distinguished vector superbundles . . . . . . . . . . . . . . . . . 8

3 Nonlinear Connections in DVS-Bundles 12

3.1 N-connections in vs-bundles . . . . . . . . . . . . . . . . . . . . 13

$3.2 \mathrm{~N}$-connections in dvs-bundles . . . . . . . . . . . . . . . . . . 15

4 Geometric Objects in DVS-Bundles 20

$4.1 \quad$ D-tensors and d-connections in dvs-bundles . . . . . . . . . . . 20

4.2 Torsions and curvatures of d-connections . . . . . . . . . . . . 22

4.3 Bianchi and Ricci identities . . . . . . . . . . . . . . . . 26

4.4 Cartan structure equations in dvs-bundles . . . . . . . . . . . . 30

4.5 Metrics in dvs-bundles . . . . . . . . . . . . . . . . . . . . . . . . . 31

5 Higher Order Tangent S-bundles 34

5.1 Supersymmetric extensions of Finsler spaces . . . . . . . . . . . 35

5.2 Higher order prolongations of Riemann,Finsler and Lagrange s-

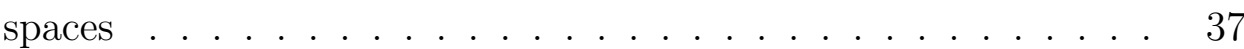

$5.3 \quad$ Higher order Lagrange s-spaces . . . . . . . . . . . . . . . . . . 39

6 Superstrings in Higher Order Anisotropic S-Spaces 39

6.1 Two dimensional higher order anisotropic sigma s-models . . . . 39

6.2 Locally anisotropic heterotic strings . . . . . . . . . . . . . . . . 44 
7 Background D-Field Methods for $\sigma$-Models $\quad 47$

8 Green-Schwarz Action in DVS-Bundles 54

9 Fermi Strings in Higher Order Anisotropic Spaces 59

10 Anomalies in Locally Anisotropic $\sigma$-Models 65

10.1 One-loop calculus . . . . . . . . . . . . . . 65

10.2 Two-loop calculus $\ldots \ldots \ldots \ldots \ldots$

\begin{tabular}{ll}
\hline 11 Discussion and Conclusions & 71
\end{tabular}

\section{Introduction}

The differential supergeometry has been formulated with the aim of getting a geometric framework for the supersymmetric field theories (see the theory of graded manifolds [19, 66, 67, 65], the theory of supermanifolds [126, 88, [13, 58] and, for detailed considerations of geometric and topological aspects of supermanifolds and formulation of superanalysis, [33, 26, 72, 53, 120, 122]). In this work we apply the supergeometric formalism for a study of a new class of (higher order anisotropic) superspaces.

The concept of local anisotropy is largely used in some divisions of theoretical and mathematical physics [121, 56, 57, 78] (see also possible applications in physics and biology in [6, 5]). The first models of locally anisotropic (la) spaces (la-spaces) have been proposed by P.Finsler [38] and E.Cartan [29] (early approaches and modern treatments of Finsler geometry and its extensions can be found, for instance, in [90, 7, 8, 74]). In our works [102, 103, 104, 106, 109, 113, 119, 116] we try to formulate the geometry of la-spaces in a manner as to include both variants of Finsler and Lagrange, in general supersymmetric, extensions and higher dimensional Kaluza-Klein (super)spaces as well to propose general principles and methods of construction of models of classical and quantum field interactions and stochastic processes on spaces with generic anisotropy.

We cite here the works [15, 16] by A. Bejancu where a new viewpoint on differential geometry of supermanifolds is considered. The author introduced the nonlinear connection ( $\mathrm{N}$-connection) structure and developed a corresponding distinguished by $\mathrm{N}$-connection supertensor covariant differential calculus in the frame of De Witt [126] approach to supermanifolds in the framework of the geometry of superbundles with typical fibres parametrized by noncommutative coordinates. This was the first example of superspace with local anisotropy. In our turn we have given a general definition of locally anisotropic superspaces 
(la-superspaces) [106]. It should be noted here that in our supersymmetric generalizations we were inspired by the R.Miron, M. Anastasiei and Gh. Atanasiu works on the geometry of nonlinear connections in vector bundles and higher order Lagrange spaces [75, 76, 77]. In this work we shall formulate the theory of higher order vector and tangent superbundles provided with nonlinear and distinguished connections and metric structures (a generalized model of la-superspaces). Such superbundles contain as particular cases the supersymmetric extensions and various higher order prolongations of Riemann, Finsler and Lagrange spaces.

The superstring theory holds the greatest promise as the unification theory of all fundamental interactions. The superstring models contains a lot a characteristic features of Kaluza-Klein approaches, supersymmetry and supergravity, local field theory and dual models. We note that in the string theories the nonlocal one dimensional quantum objects (strings) mutually interacting by linking and separating together are considered as fundamental values. Perturbations of the quantized string are identified with quantum particles. Symmetry and conservation laws in the string and superstring theory can be considered as sweeping generalizations of gauge principles which consists the basis of quantum field models. The new physical concepts are formulated in the framework a "new" for physicists mathematical formalism of the algebraic geometry and topology [4].

The relationship between two dimensional $\sigma$-models and strings has been considered [71, 39, 27, 96, 4] in order to discuss the effective low energy field equations for the massless models of strings. Nonlinear $\sigma$-models makes up a class of quantum field systems for which the fields are also treated as coordinates of some manifolds. Interactions are introduced in a geometric manner and admit a lot of applications and generalizations in classical and quantum field and string theories. The geometric structure of nonlinear sigma models manifests the existence of topological nontrivial configuration, admits a geometric interpretation of conterterms and points to a substantial interrelation between extended supersymmetry and differential supergeometry. In connection to this a new approach based on nonlocal, in general, higher order anisotropic constructions seem to be emerging [101, 107, 108]. We consider the reader to be familiar with basic results from supergeometry (see, for instance, [33, 67, 126, 88]), supergravity theories [41, 92, 125, 123, 124, and superstrings [54, 95, 63, 64,

In this work we shall present an introduction into the theory of higher order anisotropic superstrings being a natural generalization to locally anisotropic (la) backgrounds (we shall write in brief la-backgrounds, la-spaces and la-geometry) of the Polyakov's covariant functional-integral approach to string theory [85]. Our aim is to show that a corresponding low-energy string dynamics contains the motion equations for field equations on higher order anisotropic superspaces 
and to analyze the geometry of the perturbation theory of the locally anisotropic supersymmetric sigma models. We note that this work is devoted to supersymmetric models of locally anisotropic superstrings.

The work is organized as follows: Section 2 contains a brief review on supermanifolds and superbundles and an introduction into the geometry of higher order distinguished vector superbundles. Section 3 deals with the geometry of nonlinear and linear distinguished connections in vector superbundles and distinguished vector superbundles. The geometry of the total space of distinguished vector superbundles is studied in section 4; distinguished connection and metric structures, their torsions, curvatures and structure equations are considered. Generalized Lagrange and Finsler superspaces and thears higher order prolongations are defined in section 5. Section 6 contains an introduction into the geometry of two dimensional higher order anisotropic sigma models and an locally anisotropic approach to heterotic strings. In section 7 the background field method for $\sigma$-models is generalized for a distinguished calculus locally adapted to the $\mathrm{N}$-connection structure in higher order anisotropic superspaces. Section 8 is devoted to a study of Green-Schwartz action in distinguished vector superbundles. Fermi strings in higher order anisotropic spaces are considered in section 9. An example of one-loop and two-loop calculus for anomalies of locally anisotropic strings is presented in section 10. A discussion and conclusions are drawn in section 11.

\section{Distinguished Superbundles}

In this section we establish the necessary terminology on supermanifolds ( $\mathrm{s}^{-}$ manifolds ) 126, 88, 89, 58, 120, 53, 72, 13, 26, 33, 53 and present an introduction into the geometry of distinguished vector superbundles (dvs-bundles) [110]. Here we note that a number of different approaches to supermanifolds are broadly equivalent for local considerations. For simplicity, we shall restrict our study only with geometric constructions on locally trivial superspaces.

\subsection{Supermanifolds and superbundles}

To build up s-manifolds (see [88, 58, 120]) one uses as basic structures Grassmann algebra and Banach space. A Grassmann algebra is introduced as a real associative algebra $\Lambda$ (with unity) possessing a finite (canonical) set of anticommutative generators $\beta_{\hat{A}},\left[\beta_{\hat{A}}, \beta_{\hat{B}}\right]_{+}=\beta_{\hat{A}} \beta_{\hat{C}}+\beta_{\hat{C}} \beta_{\hat{A}}=0$, where $\hat{A}, \hat{B}, \ldots=$ $1,2, \ldots, \hat{L}$. In this case it is also defined a $Z_{2}$-graded commutative algebra $\Lambda_{0}+\Lambda_{1}$, whose even part $\Lambda_{0}$ (odd part $\Lambda_{1}$ ) is a $2^{\hat{L}-1}$-dimensional real vector space of even (odd) products of generators $\beta_{\hat{A}}$. After setting $\Lambda_{0}=\mathcal{R}+\Lambda_{0}{ }^{\prime}$, where $\mathcal{R}$ is the 
real number field and $\Lambda_{0}{ }^{\prime}$ is the subspace of $\Lambda$ consisting of nilpotent elements, the projections $\sigma: \Lambda \rightarrow \mathcal{R}$ and $s: \Lambda \rightarrow \Lambda_{0}{ }^{\prime}$ are called, respectively, the body and soul maps.

A Grassmann algebra can be provided with both structures of a Banach algebra and Euclidean topological space by the norm 88

$$
\|\xi\|=\Sigma_{\hat{A}_{i}}\left|a^{\hat{A}_{1} \ldots \hat{A}_{k}}\right|, \xi=\Sigma_{r=0}^{\hat{L}} a^{\hat{A}_{1} \ldots \hat{A}_{r}} \beta_{\hat{A}_{1} \ldots \beta_{\hat{A}_{r}}} .
$$

A superspace is introduced as a product

$$
\Lambda^{n, k}=\underbrace{\Lambda_{0} \times \ldots \times \Lambda_{0}}_{n} \times \underbrace{\Lambda_{1} \times \ldots \times \Lambda_{1}}_{k}
$$

which is the $\Lambda$-envelope of a $Z_{2}$-graded vector space $V^{n, k}=V_{0} \otimes V_{1}=\mathcal{R}^{n} \oplus \mathcal{R}^{k}$ is obtained by multiplication of even (odd) vectors of $V$ on even (odd) elements of $\Lambda$. The superspace (as the $\Lambda$-envelope) posses $(n+k)$ basis vectors $\left\{\hat{\beta}_{i}, \quad i=\right.$ $0,1, \ldots, n-1$, and $\left.\beta_{\hat{i}}, \quad \hat{i}=1,2, \ldots k\right\}$. Coordinates of even (odd) elements of $V^{n, k}$ are even (odd) elements of $\Lambda$. We can consider equivalently a superspace $V^{n, k}$ as a $\left(2^{\hat{L}-1}\right)(n+k)$-dimensional real vector spaces with a basis $\left\{\hat{\beta}_{i(\Lambda)}, \beta_{\hat{i}(\Lambda)}\right\}$.

Functions of superspaces, differentiation with respect to Grassmann coordinates, supersmooth (superanalytic) functions and mappings are introduced by analogy with the ordinary case, but with a glance to certain specificity caused by changing of real (or complex) number field into Grassmann algebra $\Lambda$. Here we remark that functions on a superspace $\Lambda^{n, k}$ which takes values in Grassmann algebra can be considered as mappings of the space $\mathcal{R}^{\left(2^{(\hat{L}-1)}\right)(n+k)}$ into the space $\mathcal{R}^{2 \hat{L}}$. Functions differentiable on Grassmann coordinates can be rewritten via derivatives on real coordinates, which obey a generalized form of CauchyRiemann conditions.

A $(n, k)$-dimensional s-manifold $\tilde{M}$ can be defined as a Banach manifold (see, for example, 68 modeled on $\Lambda^{n, k}$ endowed with an atlas $\psi=\left\{U_{(i)}, \psi_{(i)}\right.$ : $\left.U_{(i)} \rightarrow \Lambda^{n, k},(i) \in J\right\}$ whose transition functions $\psi_{(i)}$ are supersmooth [88, 58]. Instead of supersmooth functions we can use $G^{\infty}$-functions 88 and introduce $G^{\infty}$-supermanifolds ( $G^{\infty}$ denotes the class of superdifferentiable functions). The local structure of a $G^{\infty}$-supermanifold is built very much as on a $C^{\infty}$-manifold. Just as a vector field on a $n$-dimensional $C^{\infty}$-manifold written locally as

$$
\sum_{i=0}^{n-1} \quad f_{i}\left(x^{j}\right) \frac{\partial}{\partial x^{i}}
$$

where $f_{i}$ are $C^{\infty}$-functions, a vector field on an $(n, k)$-dimensional $G^{\infty}$-supermanifold $\tilde{M}$ can be expressed locally on an open region $U \subset \tilde{M}$ as

$$
\sum_{I=0}^{n-1+k} \quad f_{I}\left(x^{J}\right) \frac{\partial}{\partial x^{I}}=\Sigma_{i=0}^{n-1} \quad f_{i}\left(x^{j}, \theta^{\hat{j}}\right) \frac{\partial}{\partial x^{i}}+\sum_{\hat{i}=1}^{k} \quad f_{\hat{i}}\left(x^{j}, \theta^{\hat{j}}\right) \frac{\partial}{\partial \theta^{\hat{i}}},
$$


where $x=(\hat{x}, \theta)=\left\{x^{I}=\left(\hat{x}^{i}, \theta^{\hat{i}}\right)\right\}$ are local (even, odd) coordinates. We shall use indices $I=(i, \hat{i}), J=(j, \hat{j}), K=(k, \hat{k}), \ldots$ for geometric objects on $\tilde{M}$. A vector field on $U$ is an element $X \subset E n d\left[G^{\infty}(U)\right]$ (we can also consider supersmooth functions instead of $G^{\infty}$-functions) such that

$$
X(f g)=(X f) g+(-)^{|f||X|} f X g,
$$

for all $f, g$ in $G^{\infty}(U)$, and

$$
X(a f)=(-)^{|X||a|} a X f,
$$

where $|X|$ and $|a|$ denote correspondingly the parity $(=0,1)$ of values $X$ and $a$ and for simplicity in this work we shall write $(-)^{|f||X|}$ instead of $(-1)^{|f||X|}$.

A super Lie group (sl-group) 89] is both an abstract group and a s-manifold, provided that the group composition law fulfills a suitable smoothness condition (i.e. to be superanalytic, for short, $s a$ [58).

In our further considerations we shall use the group of automorphisms of $\Lambda^{(n, k)}$, denoted as $G L(n, k, \Lambda)$, which can be parametrized as the super Lie group of invertible matrices

$$
Q=\left(\begin{array}{cc}
A & B \\
C & D
\end{array}\right)
$$

where $\mathrm{A}$ and $\mathrm{D}$ are respectively $(n \times n)$ and $(k \times k)$ matrices consisting of even Grassmann elements and $\mathrm{B}$ and $\mathrm{C}$ are rectangular matrices consisting of odd Grassmann elements. A matrix $\mathrm{Q}$ is invertible as soon as maps $\sigma A$ and $\sigma D$ are invertible matrices. A sl-group represents an ordinary Lie group included in the group of linear transforms $G L\left(2^{\hat{L}-1}(n+k), \mathcal{R}\right)$. For matrices of type Q one defines [19, 66, 67] the superdeterminant, $\operatorname{sdet} Q$, supertrace, $\operatorname{str} Q$, and superrank, srankQ.

A Lie superalgebra (sl-algebra) is a $Z_{2}$-graded algebra $A=A_{0} \oplus A_{1}$ endowed with product $[$,$\} satisfying the following properties:$

$$
\begin{gathered}
{\left[I, I^{\prime}\right\}=-(-)^{|I|\left|I^{\prime}\right|}\left[I^{\prime}, I\right\},} \\
{\left[I,\left[I^{\prime}, I^{\prime \prime}\right\}\right\}=\left[\left[I, I^{\prime}\right\}, I^{\prime \prime}\right\}+(-)^{|I|\left|I^{\prime}\right|}\left[I^{\prime}\left[I, I^{\prime \prime}\right\}\right\},}
\end{gathered}
$$

$I \in A_{|I|}, \quad I^{\prime} \in A_{\left|I^{\prime}\right|}$, where $|I|,\left|I^{\prime}\right|=0,1$ enumerates, respectively, the possible parity of elements $I, I^{\prime}$. The even part $A_{0}$ of a sl-algebra is a usual Lie algebra and the odd part $A_{1}$ is a representation of this Lie algebra. This enables us to classify sl-algebras following the Lie algebra classification [59]. We also point out that irreducible linear representations of Lie superalgebra A are realized in $Z_{2}$-graded vector spaces by matrices $\left(\begin{array}{cc}A & 0 \\ 0 & D\end{array}\right)$ for even elements and 
$\left(\begin{array}{cc}0 & B \\ C & 0\end{array}\right)$ for odd elements and that, roughly speaking, A is a superalgebra of generators of a sl-group.

A sl-module $W$ (graded Lie module) 88 is introduced as a $Z_{2}$-graded left $\Lambda$-module endowed with a product [, $\}$ which satisfies the graded Jacobi identity and makes $W$ into a graded-anticommutative Banach algebra over $\Lambda$. One calls the Lie module $\mathrm{G}$ the set of the left-invariant derivatives of a sl-group $G$.

The tangent superbundle (ts-bundle) $T \tilde{M}$ over a s-manifold $\tilde{M}, \pi: T \tilde{M} \rightarrow$ $\tilde{M}$ is constructed in a usual manner (see, for instance, [68]) by taking as the typical fibre the superspace $\Lambda^{n, k}$ and as the structure group the group of automorphisms, i.e. the sl-group $G L(n, k, \Lambda)$.

Let us denote by $\mathcal{F}$ a vector superspace ( vs-space) of dimension $(m, l)$ (with respect to a chosen base we parametrize an element $y \in \mathcal{E}$ as $y=(\hat{y}, \zeta)=$ $\left\{y^{A}=\left(\hat{y}^{a}, \zeta^{\hat{a}}\right)\right\}$, where $a=1,2, \ldots, m$ and $\left.\hat{a}=1,2, \ldots, l\right)$. We shall use indices $A=(a, \hat{a}), B=(b, \hat{b}), \ldots$ for objects on vs-spaces. A vector superbundle ( vsbundle ) $\tilde{\mathcal{E}}$ over base $\tilde{M}$ with total superspace $\tilde{E}$, standard fibre $\hat{\mathcal{F}}$ and surjective projection $\pi_{E}: \tilde{E} \rightarrow \tilde{M}$ is defined (see details and variants in [26, 122]) as in the case of ordinary manifolds (see, for instance, [68, 75, 76]). A section of $\tilde{\mathcal{E}}$ is a supersmooth map $s: U \rightarrow \tilde{E}$ such that $\pi_{E} \cdot s=i d_{U}$.

A subbundle of $\tilde{\mathcal{E}}$ is a triple $\left(\tilde{\mathcal{B}}, f, f^{\prime}\right)$, where $\tilde{\mathcal{B}}$ is a vs-bundle on $\tilde{M}$, maps $f: \tilde{\mathcal{B}} \rightarrow \tilde{\mathcal{E}}$ and $f^{\prime}: \tilde{M} \rightarrow \tilde{M}$ are supersmooth, and $(i) \quad \pi_{E} \circ f=f^{\prime} \circ \pi_{B} ;(i i) \quad f:$ $\pi_{B}^{-1}(x) \rightarrow \pi_{E}^{-1} \circ f^{\prime}(x)$ is a vs-space homomorphism. We denote by

$$
u=(x, y)=(\hat{x}, \theta, \hat{y}, \zeta)=\left\{u^{\alpha}=\left(x^{I}, y^{A}\right)=\left(\hat{x}^{i}, \theta^{\hat{i}}, \hat{y}^{a}, \zeta^{\hat{a}}\right)=\left(\hat{x}^{i}, x^{\hat{i}}, \hat{y}^{a}, y^{\hat{a}}\right)\right\}
$$

the local coordinates in $\tilde{\mathcal{E}}$ and write their transformations as

$$
x^{I^{\prime}}=x^{I^{\prime}}\left(x^{I}\right), \quad \operatorname{srank}\left(\frac{\partial x^{I^{\prime}}}{\partial x^{I}}\right)=(n, k),
$$

$y^{A^{\prime}}=Y_{A}^{A^{\prime}}(x) y^{A}$, where $Y_{A}^{A^{\prime}}(x) \in G(m, l, \Lambda)$.

For local coordinates and geometric objects on ts-bundle $T \tilde{M}$ we shall not distinguish indices of coordinates on the base and in the fibre and write, for instance,

$$
u=(x, y)=(\hat{x}, \theta, \hat{y}, \zeta)=\left\{u^{\alpha}=\left(x^{I}, y^{I}\right)=\left(\hat{x}^{i}, \theta^{\hat{i}}, \hat{y}^{i}, \zeta^{\hat{i}}\right)=\left(\hat{x}^{i}, x^{\hat{i}}, \hat{y}^{i}, y^{\hat{i}}\right)\right\} .
$$

We shall use Greek indices for marking local coordinates on both s-vector and usual vector bundles.

\subsection{Distinguished vector superbundles}

Some recent considerations in mathematical physics are based on the so-called $\mathrm{k}$-jet spaces (see, for instance, 994, 93, 9]). In order to formulate a systematic 
theory of connections and of geometric structures on $\mathrm{k}$-jet bundles, in a manner following the approaches [128] and [75, 76] R. Miron and Gh. Atanasiu [77] introduced the concept of $\mathrm{k}$-osculator bundle for which a fiber of $\mathrm{k}$-jets is changed into a k-osculator fiber representing an element of $\mathrm{k}$-order curve. Such considerations are connected with geometric constructions on tangent bundles of higher order. On the other hand for developments in modern supersymmetric Kaluza-Klein theories (see, for instance, [92]) a substantial interest would present a variant of "osculator" space for which the higher order tangent sspace distributions are of different dimensions. The second part of this section is devoted to the definition of such type distinguished vector superbundle spaces.

A vector superspace $\mathcal{F}^{<z>}$ of dimension $(m, l)$ is a distinguished vector superspace ( dvs-space ) if it is decomposed into an invariant oriented direct sum $\mathcal{F}^{<z>}=\mathcal{F}_{(1)} \oplus \mathcal{F}_{(2)} \oplus \ldots \oplus \mathcal{F}_{(z)}$ of vs-spaces $\mathcal{F}_{(p)}, \operatorname{dim} \mathcal{F}_{(p)}=\left(m_{(p)}, l_{(p)}\right)$, where $(p)=(1),(2), \ldots,(z), \sum_{p=1}^{p=z} m_{(p)}=m, \sum_{p=1}^{p=z} l_{(p)}=l$.

Coordinates on $\mathcal{F}^{<p>}$ will be parametrized as

$$
\begin{gathered}
y^{<p>}=\left(y_{(1)}, y_{(2)}, \ldots, y_{(p)}\right)=\left(\hat{y}_{(1)}, \zeta_{(1)}, \hat{y}_{(2)}, \zeta_{(2)}, \ldots, \hat{y}_{(p)}, \zeta_{(p)}\right)= \\
\left\{y^{<A>}=\left(\hat{y}^{<a>}, \zeta^{<\hat{a}>}\right)=\left(\hat{y}^{<a>}, y^{<\hat{a}>}\right)\right\}
\end{gathered}
$$

where bracketed indices are correspondingly split on $\mathcal{F}_{(p)}$-components:

$$
\begin{gathered}
<A>=\left(A_{(1)}, A_{(2)}, \ldots, A_{(p)}\right),<a>=\left(a_{(1)}, a_{(2)}, \ldots, a_{(p)}\right) \\
\text { and }<\widehat{a}>=\left(\widehat{a}_{(1)} \widehat{a}_{(2)}, \ldots, \widehat{a}_{(p)}\right),
\end{gathered}
$$

For simplicity, we shall also write $(2)$ as $<A>=\left(A_{1}, A_{2}, \ldots, A_{p}\right),\langle a\rangle=$ $\left(a_{1}, a_{2}, \ldots, a_{p}\right)$ and $<\widehat{a}>=\left(\widehat{a}_{1} \widehat{a}_{2}, \ldots, \widehat{a}_{p}\right)$ if this will give not rise to ambiguities.

A distinguished vector superbundle (dvs-bundle ) $\widetilde{\mathcal{E}}^{<z>}=\left(\tilde{E}^{<z>}, \pi^{<d>}, \mathcal{F}^{<d>}, \tilde{M}\right)$, with surjective projection $\pi^{<z>}: \tilde{E}^{<z>} \rightarrow \tilde{M}$, where $\tilde{M}$ and $\tilde{E}^{<z>}$ are respectively base and total s-spaces and the dvs-space $\mathcal{F}^{<z>}$ is the standard fibre.

A dvs-bundle $\tilde{\mathcal{E}}^{<z>}$ is constructed as an oriented set of vs-bundles $\pi^{<p>}$ : $\tilde{E}^{<p>} \rightarrow \tilde{E}^{<p-1>}$ (with typical fiber $\left.\mathcal{F}^{<p>}, p=1,2, \ldots, z\right) ; \tilde{E}^{<0>}=\tilde{M}$. We shall use index $z(p)$ as to denote the total (intermediate) numbers of consequent vs-bundle coverings of $\tilde{M}$.

Local coordinates on $\widetilde{\mathcal{E}}^{<p>}$ are denoted as

$$
\begin{gathered}
u_{(p)}=\left(x, y_{<p>}\right)=\left(x, y_{(1)}, y_{(2)}, \ldots, y_{(p)}\right)= \\
\left(\hat{x}, \theta, \hat{y}_{<p>}, \zeta_{<p>}\right)=\left(\hat{x}, \theta, \hat{y}_{(1)}, \zeta_{(1)}, \hat{y}_{(2)}, \zeta_{(2)}, \ldots, \hat{y}_{(p)}, \zeta_{(p)}\right)= \\
\left\{u^{<\alpha>}=\left(x^{I}, y^{<A>}\right)=\left(\hat{x}^{i}, \theta^{\hat{i}}, \hat{y}^{<a>}, \zeta^{<\hat{a}>}\right)=\left(\hat{x}^{i}, x^{\hat{i}}, \hat{y}^{<a>}, y^{<\hat{a}>}\right)=\right.
\end{gathered}
$$




$$
\left.\left(x^{I}=y^{A_{0}}, y^{A_{1}}, \ldots, y^{A_{p}}, \ldots, y^{A_{z}}\right)\right\}
$$

(in our further considerations we shall consider different variants of splitting of indices of geometric objects).

Instead of (1) the coordinate transforms for dvs-bundles $\left\{u^{<\alpha>}=\left(x^{I}, y^{<A>}\right)\right\} \rightarrow\left\{u^{<\alpha^{\prime}>}=\left(x^{I^{\prime}}, y^{<A^{\prime}>}\right)\right\}$ are given by recurrent maps:

$$
\begin{aligned}
& x^{I^{\prime}}=x^{I^{\prime}}\left(x^{I}\right), \quad \operatorname{srank}\left(\frac{\partial x^{I^{\prime}}}{\partial x^{I}}\right)=(n, k), \\
& y_{(1)}^{A_{1}^{\prime}}=K_{A_{1}}^{A_{1}^{\prime}}(x) y_{(1)}^{A_{1}}, K_{A_{1}}^{A_{1}^{\prime}}(x) \in G\left(m_{(1)}, l_{(1)}, \Lambda\right), \\
& y_{(p)}^{A_{p}^{\prime}}=K_{A_{p}}^{A_{p}^{\prime}}\left(u_{(p-1)}\right) y_{(p)}^{A_{p}}, K_{A_{p}}^{A_{p}^{\prime}}\left(u_{(p-1)}\right) \in G\left(m_{(p)}, l_{(p)}, \Lambda\right), \\
& y_{(z)}^{A_{z}^{\prime}}=K_{A_{z}}^{A_{z}^{\prime}}\left(u_{(z-1)}\right) y_{(z)}^{A_{z}}, K_{A_{z}}^{A_{z}^{\prime}}\left(u_{(z-1)}\right) \in G\left(m_{(z)}, l_{(z)}, \Lambda\right) .
\end{aligned}
$$

In brief we write transforms (3) as

$$
x^{I^{\prime}}=x^{I^{\prime}}\left(x^{I}\right), y^{<A^{\prime}>}=K_{<A>}^{<A^{\prime}>} y^{<A>} .
$$

More generally, we shall consider matrices $K_{<\alpha>}^{<\alpha^{\prime}>}=\left(K_{I}^{I^{\prime}}, K_{<A>}^{<A^{\prime}>}\right)$, where $K_{I}^{I^{\prime}} \doteq$ $\frac{\partial x^{I^{\prime}}}{\partial x^{I}}$.

In consequence the local coordinate bases of the module of ds-vector fields $\Xi\left(\widetilde{\mathcal{E}}^{<z>}\right)$,

$$
\begin{gathered}
\partial_{<\alpha>}=\left(\partial_{I}, \partial_{<A>}\right)=\left(\partial_{I}, \partial_{\left(A_{1}\right)}, \partial_{\left(A_{2}\right)}, \ldots, \partial_{\left(A_{z}\right)}\right)= \\
\frac{\partial}{\partial u^{<\alpha>}}=\left(\frac{\partial}{\partial x^{I}}, \frac{\partial}{\partial y_{(1)}^{A_{1}}}, \frac{\partial}{\partial y_{(2)}^{A_{2}}}, \ldots, \frac{\partial}{\partial y_{(z)}^{A_{z}}}\right)
\end{gathered}
$$

(the dual coordinate bases are denoted as

$$
\begin{gathered}
d^{<\alpha>}=\left(d^{I}, d^{<A>}\right)=\left(d^{I}, d^{\left(A_{1}\right)}, d^{\left(A_{2}\right)}, \ldots, d^{\left(A_{z}\right)}\right)= \\
\left.d u^{<\alpha>}=\left(d x^{I}, d y^{\left(A_{1}\right)}, d y^{\left(A_{2}\right)}, \ldots, d y^{\left(A_{z}\right)}\right) \quad\right)
\end{gathered}
$$

are transformed as

$$
\begin{gathered}
\partial_{<\alpha>}=\left(\partial_{I}, \partial_{<A>}\right)=\left(\partial_{I}, \partial_{\left(A_{1}\right)}, \partial_{\left(A_{2}\right)}, \ldots, \partial_{\left(A_{z}\right)}\right) \rightarrow \partial_{<\alpha>}= \\
\left(\partial_{I}, \partial_{<A>}\right)=\left(\partial_{I}, \partial_{\left(A_{1}\right)}, \partial_{\left(A_{2}\right)}, \ldots, \partial_{\left(A_{z}\right)}\right) \\
\frac{\partial}{\partial x^{I}}=K_{I}^{I^{\prime}} \frac{\partial}{\partial x^{I^{\prime}}}+Y_{(1,0) I}^{A_{1}^{\prime}} \frac{\partial}{\partial y_{(1)}^{A_{1}^{\prime}}}+Y_{(2,0) I}^{A_{2}^{\prime}} \frac{\partial}{\partial y_{(2)}^{A_{2}^{\prime}}}+\ldots+Y_{(z, 0) I}^{A_{z}^{\prime}} \frac{\partial}{\partial y_{(z)}^{A_{z}^{\prime}}}
\end{gathered}
$$




$$
\begin{gathered}
\frac{\partial}{\partial y_{(1)}^{A_{1}}}=K_{A_{1}}^{A_{1}^{\prime}} \frac{\partial}{\partial y_{(1)}^{A_{1}^{\prime}}}+Y_{(2,1) A_{1}}^{A_{2}^{\prime}} \frac{\partial}{\partial y_{(2)}^{A_{2}^{\prime}}}+\ldots+Y_{(z, 1) A_{1}}^{A_{1}^{\prime}} \frac{\partial}{\partial y_{(z)}^{A_{z}^{\prime}}}, \\
\frac{\partial}{\partial y_{(2)}^{A_{2}}}=K_{A_{2}}^{A_{2}^{\prime}} \frac{\partial}{\partial y_{(2)}^{A_{2}^{\prime}}}+Y_{(3,2) A_{2}}^{A_{3}^{\prime}} \frac{\partial}{\partial y_{(3)}^{A_{3}^{\prime}}}+\ldots+Y_{(z, 2) A_{2}}^{A_{z}^{\prime}} \frac{\partial}{\partial y_{(z)}^{A_{z}^{\prime}}}, \\
\frac{\partial}{\partial y_{(z-1)}^{A_{z-1}}}=K_{A_{z-1}}^{A_{z-1}^{\prime}} \frac{\partial}{\partial y_{(z-1)}^{A_{z-1}^{\prime}}}+Y_{(z, z-1) A_{s-1}}^{A_{1}^{\prime}} \frac{\partial}{\partial y_{(z)}^{A_{z}^{\prime}}}, \\
\frac{\partial}{\partial y_{(z)}^{A_{z}}}=K_{A_{z}}^{A_{z}^{\prime}} \frac{\partial}{\partial y_{(z)}^{A_{z}^{\prime}}} .
\end{gathered}
$$

$Y$-matrices from (6) are partial derivations of corresponding combinations of $K$-coefficients from coordinate transforms (3),

$$
Y_{A_{f}}^{A_{p}^{\prime}}=\frac{\partial\left(K_{A_{p}}^{A_{p}^{\prime}} y^{A_{p}}\right)}{\partial y^{A_{f}}}, f<p .
$$

In brief we denote respectively ds-coordinate transforms of coordinate bases (4) and (5) as

$$
\partial_{<\alpha>}=\left(K_{<\alpha>}^{<\alpha^{\prime}>}+Y_{<\alpha>}^{<\alpha^{\prime}>}\right) \partial_{<\alpha^{\prime}>} \text { and } d^{<\alpha>}=\left(K_{<\alpha^{\prime}>}^{<\alpha>}+Y_{<\alpha^{\prime}>}^{<\alpha>}\right) d^{<\alpha^{\prime}>},
$$

where matrix $K_{<\alpha>}^{<\alpha^{\prime}>}$, its s-inverse $K_{\left\langle\alpha^{\prime}\right\rangle}^{<\alpha>}$, as well $Y_{<\alpha>}^{<\alpha^{\prime}>}$ and $Y_{\left\langle\alpha^{\prime}\right\rangle}^{<\alpha>}$ are parametrized according to (6). In order to illustrate geometric properties of some of our transforms it is useful to introduce matrix operators and to consider in explicit form the parametrizations of matrices under consideration. For instance, in operator form the transforms (6)

$$
\partial=\widehat{\mathbf{Y}} \partial^{\prime}
$$

are characterized by matrices of type

$$
\partial=\partial_{<\alpha>}=\left(\begin{array}{c}
\partial_{I} \\
\partial_{A_{1}} \\
\partial_{A_{2}} \\
\cdots \\
\partial_{A_{z}}
\end{array}\right)=\left(\begin{array}{c}
\frac{\partial}{\partial x^{I}} \\
\frac{\partial}{\partial y_{(1)}^{A}} \\
\frac{\partial}{\partial y_{(2)}^{A}} \\
\cdots \\
\frac{\partial}{\partial y_{(z)}^{A z}}
\end{array}\right), \partial^{\prime}=\partial_{<\alpha^{\prime}>}=\left(\begin{array}{c}
\partial_{I^{\prime}} \\
\partial_{A_{1}^{\prime}} \\
\partial_{A_{2}^{\prime}} \\
\cdots \\
\partial_{A_{z}^{\prime}}
\end{array}\right)=\left(\begin{array}{c}
\frac{\partial}{\partial y^{I^{\prime}}} \\
\frac{\partial y_{1}^{A_{1}^{\prime}}}{\partial} \\
\frac{\partial}{\partial y_{(2)}^{A_{2}^{\prime}}} \\
\ldots \\
\frac{\partial}{\partial y_{(z)}^{A_{z}^{\prime}}}
\end{array}\right)
$$


and

$$
\widehat{\mathbf{Y}}=\widehat{Y}_{<\alpha>}^{<\alpha^{\prime}>}=\left(\begin{array}{ccccc}
K_{I}^{I^{\prime}} & Y_{(1,0) I}^{A_{1}^{\prime}} & Y_{(2,0) I}^{A_{2}^{\prime}} & \ldots & Y_{(z, 0) I}^{A_{z}^{\prime}} \\
0 & K_{A_{1}}^{A_{1}^{\prime}} & Y_{(2,1) A_{1}}^{A_{2}^{\prime}} & \ldots & Y_{(z, 1) A_{1}}^{A_{z}^{\prime}} \\
0 & 0 & K_{A_{2}}^{A_{2}^{\prime}} & \ldots & Y_{(z, 2) A_{2}}^{A_{z}^{\prime}} \\
\ldots & \ldots & \ldots & \ldots & \ldots \\
0 & 0 & 0 & \ldots & K_{A_{z}}^{A_{z}^{\prime}}
\end{array}\right)
$$

We note that we obtain a supersimmetric generalization of the MironAtanasiu [77] osculator bundle $\left(\operatorname{Osc}^{z} \tilde{M}, \pi, \tilde{M}\right)$ if the fiber space is taken to be a direct sum of $z$ vector s-spaces of the same $\operatorname{dimension} \operatorname{dim} \mathcal{F}=\operatorname{dim} \widetilde{M}$, i.e. $\mathcal{F}^{<d>}=\mathcal{F} \oplus \mathcal{F} \oplus \ldots \oplus \mathcal{F}$. In this case the $K$ and $Y$ matrices from (3) and (6) satisfy identities:

$$
\begin{gathered}
K_{A_{1}}^{A_{1}^{\prime}}=K_{A_{2}}^{A_{2}^{\prime}}=\ldots=K_{A_{z}}^{A_{z}^{\prime}}, \\
Y_{(1,0) A}^{A^{\prime}}=Y_{(2,1) A}^{A^{\prime}}=\ldots=Y_{(z, z-1) A}^{A^{\prime}}, \\
\cdots \ldots \ldots \ldots \ldots \ldots \ldots \ldots \ldots \ldots \ldots \ldots \ldots \ldots \ldots \ldots \ldots \\
Y_{(p, 0) A}^{A^{\prime}}=Y_{(p+1,1) A}^{A^{\prime}}=\ldots=Y_{(z, z-1) A}^{A^{\prime}}, \quad(p=2, \ldots, z-1) .
\end{gathered}
$$

For $s=1$ the $O s c^{1} \widetilde{M}$ is the ts-bundle $T \widetilde{M}$.

Introducing projection $\pi_{0}^{z} \doteq \pi^{<z>}: \widetilde{\mathcal{E}}^{<z>} \rightarrow \widetilde{M}$ we can also consider projections $\pi_{p_{2}}^{p_{1}}: \widetilde{\mathcal{E}}^{<p_{1}>} \rightarrow \widetilde{\mathcal{E}}^{<p_{2}>} \quad\left(p_{2}<p_{1}\right)$ defined as

$$
\pi_{s_{2}}^{s_{1}}\left(x, y^{(1)}, \ldots, y^{\left(p_{1}\right)}\right)=\left(x, y^{(1)}, \ldots, y^{\left(p_{2}\right)}\right)
$$

The s-differentials $d \pi_{p_{2}}^{p_{1}}: T\left(\widetilde{\mathcal{E}}^{<p_{1}>}\right) \rightarrow T\left(\widetilde{\mathcal{E}}^{<p_{2}>}\right)$ of maps $\pi_{p_{2}}^{p_{1}}$ in turn define vertical dvs-subbundles $V_{h+1}=\operatorname{Kerd} \pi_{h}^{p_{1}}\left(h=0,1, \ldots, p_{1}-1\right)$ of the tangent dvsbundle $T\left(\widetilde{\mathcal{E}}^{<z>}\right)$ ( the dvs-space $V_{1}=V$ is the vertical dvs-subbundle on $\widetilde{\mathcal{E}}^{<z>}$. The local fibres of dvs-subbundles $V_{h}$ determines this regular s-distribution $V_{h+1}: u \in \widetilde{\mathcal{E}}^{<z>} \rightarrow V_{h+1}(u) \subset T\left(\widetilde{\mathcal{E}}^{<z>}\right)$ for which one holds inclusions $V_{z} \subset$ $V_{z-1} \subset \ldots \subset V_{1}$. The enumerated properties of vertical dvs-subbundles are explicitly illustrated by transformation laws (6) for distinguished local bases.

\section{Nonlinear Connections in DVS-Bundles}

The purpose of this section is to present an introduction into geometry of the nonlinear connection structures in dvs-bundles. The concept of nonlinear connection ( $\mathrm{N}$-connection ) was introduced in the framework of Finsler geometry [30, 29, 60] (the global definition of $\mathrm{N}$-connection is given in [12]). It should be noted here that the $\mathrm{N}$-connection ( splitting ) field could play an important rule in modeling various variants of dynamical reduction from higher dimensional to 
lower dimensional s-spaces with (or not) different types of local anisotropy. In monographs [75, 76] there are contained detailed investigations of geometrical properties of N-connection structures in $\mathrm{v}$-bundles and different generalizations of Finsler geometry and some proposals (see Chapter XII in [75, written by S. Ikeda) on physical interpretation of $\mathrm{N}$-connection in the framework of "unified" field theory with interactions nonlocalized by $\mathrm{y}$-dependencies are discussed. We emphasize that $\mathrm{N}$-connection is a different geometrical object from that introduced by using nonlinear realizations of gauge groups and supergroups (see, for instance, the collection of works on supergravity [92] and approaches to gauge gravity [98, 86]).To make the presentation to aid rapid assimilation we shall have realized our geometric constructions firstly for vs-bundles then we shall extend them for higher order extensions, i.e. for general dvs-bundles.

\subsection{N-connections in vs-bundles}

Let consider the definitions of $\mathrm{N}$-connection structure 106 in a vs-bundle $\tilde{\mathcal{E}}=$ $\left(\tilde{E}, \pi_{E}, \tilde{M}\right)$ whose type fibre is $\hat{\mathcal{F}}$ and $\pi^{T}: T \tilde{\mathcal{E}} \rightarrow T \tilde{M}$ is the superdifferential of the map $\pi_{E}\left(\pi^{T}\right.$ is a fibre-preserving morphism of the ts-bundle $\left(T \tilde{\mathcal{E}}, \tau_{E}, \tilde{M}\right)$ to $\tilde{E}$ and of ts-bundle $(T \tilde{M}, \tau, \tilde{M})$ to $\tilde{M})$. The kernel of this vs-bundle morphism being a subbundle of $\left(T \tilde{E}, \tau_{E}, \tilde{E}\right)$ is called the vertical subbundle over $\tilde{\mathcal{E}}$ and denoted by $V \tilde{\mathcal{E}}=\left(V \tilde{E}, \tau_{V}, \tilde{E}\right)$. Its total space is $V \tilde{\mathcal{E}}=\bigcup_{u \in \tilde{\mathcal{E}}} \quad V_{u}$, where $V_{u}=k e r \pi^{T}, \quad u \in \tilde{\mathcal{E}}$. A vector

$$
Y=Y^{\alpha} \frac{\partial}{\partial u^{\alpha}}=Y^{I} \frac{\partial}{\partial x^{I}}+Y^{A} \frac{\partial}{\partial y^{A}}=Y^{i} \frac{\partial}{\partial x^{i}}+Y^{\hat{i}} \frac{\partial}{\partial \theta^{\hat{i}}}+Y^{a} \frac{\partial}{\partial y^{a}}+Y^{\hat{a}} \frac{\partial}{\partial \zeta^{\hat{a}}}
$$

tangent to $\tilde{\mathcal{E}}$ in the point $u \in \tilde{\mathcal{E}}$ is locally represented as

$$
(u, Y)=\left(u^{\alpha}, Y^{\alpha}\right)=\left(x^{I}, y^{A}, Y^{I}, Y^{A}\right)=\left(\hat{x}^{i}, \theta^{\hat{i}}, \hat{y}^{a}, \zeta^{\hat{a}}, \hat{Y}^{i}, Y^{\hat{i}}, \hat{Y}^{a}, Y^{\hat{a}}\right) .
$$

A nonlinear connection, $\mathrm{N}$-connection, in vs-bundle $\tilde{\mathcal{E}}$ is a splitting on the left of the exact sequence

$$
0 \longmapsto V \tilde{\mathcal{E}} \stackrel{i}{\longmapsto} T \tilde{\mathcal{E}} \longmapsto T \tilde{\mathcal{E}} / V \tilde{\mathcal{E}} \longmapsto 0
$$

i.e. a morphism of vs-bundles $N: T \tilde{\mathcal{E}} \in V \tilde{\mathcal{E}}$ such that $N \circ i$ is the identity on $V \tilde{\mathcal{E}}$.

The kernel of the morphism $N$ is called the horizontal subbundle and denoted by

$$
\left(H \tilde{E}, \tau_{E}, \tilde{E}\right) .
$$

From the exact sequence (7) one follows that $\mathrm{N}$-connection structure can be equivalently defined as a distribution $\left\{\tilde{E}_{u} \rightarrow H_{u} \tilde{E}, T_{u} \tilde{E}=H_{u} \tilde{E} \oplus V_{u} \tilde{E}\right\}$ on $\tilde{E}$ 
defining a global decomposition, as a Whitney sum,

$$
T \tilde{\mathcal{E}}=H \tilde{\mathcal{E}}+V \tilde{\mathcal{E}}
$$

To a given $\mathrm{N}$-connection we can associate a covariant s-derivation on $\tilde{M}$ :

$$
\nabla_{X} Y=X^{I}\left\{\frac{\partial Y^{A}}{\partial x^{I}}+N_{I}^{A}(x, Y)\right\} s_{A},
$$

where $s_{A}$ are local independent sections of $\tilde{\mathcal{E}}, \quad Y=Y^{A} s_{A}$ and $X=X^{I} s_{I}$.

S-differentiable functions $N_{I}^{A}$ from (3) written as functions on $x^{I}$ and $y^{A}$, $N_{I}^{A}(x, y)$, are called the coefficients of the $\mathrm{N}-$ connection and satisfy these transformation laws under coordinate transforms (1) in $\mathcal{E}$ :

$$
N_{I^{\prime}}^{A^{\prime}} \frac{\partial x^{I^{\prime}}}{\partial x^{I}}=M_{A}^{A^{\prime}} N_{I}^{A}-\frac{\partial M_{A}^{A^{\prime}}(x)}{\partial x^{I}} y^{A}
$$

If coefficients of a given $\mathrm{N}$-connection are $\mathrm{s}$-differentiable with respect to coordinates $y^{A}$ we can introduce (additionally to covariant nonlinear s-derivation (9)) a linear covariant s-derivation $\hat{D}$ (which is a generalization for vs-bundles of the Berwald connection [21]) given as follows:

$$
\hat{D}_{\left(\frac{\partial}{\partial x^{I}}\right)}\left(\frac{\partial}{\partial y^{A}}\right)=\hat{N}_{A I}^{B}\left(\frac{\partial}{\partial y^{B}}\right), \quad \hat{D}_{\left(\frac{\partial}{\partial y^{A}}\right)}\left(\frac{\partial}{\partial y^{B}}\right)=0
$$

where

$$
\hat{N}_{B I}^{A}(x, y)=\frac{\partial N_{I}^{A}(x, y)}{\partial y^{B}}
$$

and

$$
\hat{N}_{B C}^{A}(x, y)=0 \text {. }
$$

For a vector field on $\tilde{\mathcal{E}} \quad Z=Z^{I} \frac{\partial}{\partial x^{I}}+Y^{A} \frac{\partial}{\partial y^{A}}$ and $B=B^{A}(y) \frac{\partial}{\partial y^{A}}$ being a section in the vertical s-bundle $\left(V \tilde{E}, \tau_{V}, \tilde{E}\right)$ the linear connection (10) defines s-derivation (compare with (9)):

$$
\hat{D}_{Z} B=\left[Z^{I}\left(\frac{\partial B^{A}}{\partial x^{I}}+\hat{N}_{B I}^{A} B^{B}\right)+Y^{B} \frac{\partial B^{A}}{\partial y^{B}}\right] \frac{\partial}{\partial y^{A}} .
$$

Another important characteristic of a $\mathrm{N}$-connection is its curvature ( $\mathrm{N}-$ connection curvature ):

$$
\Omega=\frac{1}{2} \Omega_{I J}^{A} d x^{I} \wedge d x^{J} \otimes \frac{\partial}{\partial y^{A}}
$$


with local coefficients

$$
\Omega_{I J}^{A}=\frac{\partial N_{I}^{A}}{\partial x^{J}}-(-)^{|I J|} \frac{\partial N_{J}^{A}}{\partial x^{I}}+N_{I}^{B} \hat{N}_{B J}^{A}-(-)^{|I J|} N_{J}^{B} \hat{N}_{B I}^{A},
$$

where for simplicity we have written $(-)^{|K||J|}=(-)^{|K J|}$.

We note that linear connections are particular cases of $\mathrm{N}$-connections locally parametrized as $N_{I}^{A}(x, y)=N_{B I}^{A}(x) x^{I} y^{B}$, where functions $N_{B I}^{A}(x)$, defined on $\tilde{M}$, are called the Christoffel coefficients.

\section{$3.2 \mathrm{~N}$-connections in dvs-bundles}

In order to define a $\mathrm{N}$-connection into dvs-bundle $\widetilde{\mathcal{E}}^{<z>}$ we consider a s-subbundle $N\left(\widetilde{\mathcal{E}}^{<z>}\right)$ of the ts-bundle $T\left(\widetilde{\mathcal{E}}^{<z>}\right)$ for which one holds (see 94 and [77] respectively for jet and osculator bundles) the Whitney sum (compare with (8))

$$
T\left(\widetilde{\mathcal{E}}^{<z>}\right)=N\left(\widetilde{\mathcal{E}}^{<z>}\right) \oplus V\left(\widetilde{\mathcal{E}}^{<z>}\right) .
$$

$N\left(\widetilde{\mathcal{E}}^{<z>}\right)$ can be also interpreted as a regular s-distribution (horizontal distribution being supplementary to the vertical s-distribution $\left.V\left(\widetilde{\mathcal{E}}^{<z>}\right)\right)$ determined by maps $N: u \in \widetilde{\mathcal{E}}^{<z>} \rightarrow N(u) \subset T_{u}\left(\widetilde{\mathcal{E}}^{<z>}\right)$.

The condition of existence of a $\mathrm{N}$-connection in a dvs-bundle $\widetilde{\mathcal{E}}^{<z>}$ can be proved as in 775, 76, 77]: It is required that $\widetilde{\mathcal{E}}^{<z>}$ is a paracompact sdifferentiable (in our case) manifold.

Locally a $\mathrm{N}$-connection in $\widetilde{\mathcal{E}}^{<z>}$ is given by its coefficients

$$
\begin{gathered}
N_{(01) I}^{A_{1}}(u),\left(N_{(02) I}^{A_{2}}(u), N_{(12) A_{1}}^{A_{2}}(u)\right), \ldots\left(N_{(0 p) I}^{A_{p}}(u), N_{(1 p) A_{1}}^{A_{p}}(u), \ldots N_{(p-1 p) A_{p-1}}^{A_{p}}(u)\right), \ldots \\
\left(N_{(0 z) I}^{A_{z}}(u), N_{(1 z) A_{1}}^{A_{z}}(u), \ldots, N_{(p z) A_{p}}^{A_{z}}(u), \ldots, N_{(z-1 z) A_{z-1}}^{A_{z}}(u)\right)
\end{gathered}
$$

where, for instance, $\left(N_{(0 p) I}^{A_{p}}(u), N_{(1 p) A_{1}}^{A_{p}}(u), \ldots, N_{(p-1 p) A_{p-1}}^{A_{p}}(u)\right)$ are components of $\mathrm{N}$-connection in vs-bundle $\pi^{<p>}: \tilde{E}^{<p>} \rightarrow \tilde{E}^{<p-1>}$. Here we note that if a Nconnection structure is defined we must correlate to it the local partial derivatives on $\widetilde{\mathcal{E}}^{<z>}$ by considering instead of local coordinate bases (4) and (5) the so-called locally adapted bases ( la-bases )

$$
\begin{gathered}
\delta_{<\alpha>}=\left(\delta_{I}, \delta_{<A>}\right)=\left(\delta_{I}, \delta_{\left(A_{1}\right)}, \delta_{\left(A_{2}\right)}, \ldots, \delta_{\left(A_{s}\right)}\right)= \\
\frac{\delta}{\partial u^{<\alpha>}}=\left(\frac{\delta}{\partial x^{I}}, \frac{\delta}{\partial y_{(1)}^{A_{1}}}, \frac{\delta}{\partial y_{(2)}^{A_{2}}}, \ldots, \frac{\delta}{\partial y_{(z)}^{A_{z}}}\right)
\end{gathered}
$$


(the dual la-bases are denoted as

$$
\begin{gathered}
\delta^{<\alpha>}=\left(\delta^{I}, \delta^{<A>}\right)=\left(\delta^{I}, \delta^{\left(A_{1}\right)}, \delta^{\left(A_{2}\right)}, \ldots, \delta^{\left(A_{z}\right)}\right)= \\
\left.\delta u^{<\alpha>}=\left(\delta x^{I}, \delta y^{\left(A_{1}\right)}, \delta y^{\left(A_{2}\right)}, \ldots, \delta y^{\left(A_{z}\right)}\right) \quad\right)
\end{gathered}
$$

with components parametrized as

$$
\begin{aligned}
& \delta_{I}=\partial_{I}-N_{I}^{A_{1}} \partial_{A_{1}}-N_{I}^{A_{2}} \partial_{A_{2}}-\ldots-N_{I}^{A_{z-1}} \partial_{A_{z-1}}-N_{I}^{A_{z}} \partial_{A_{z}}, \\
& \delta_{A_{1}}=\partial_{A_{1}}-N_{A_{1}}^{A_{2}} \partial_{A_{2}}-N_{A_{1}}^{A_{3}} \partial_{A_{3}}-\ldots-N_{A_{1}}^{A_{z-1}} \partial_{A_{z-1}}-N_{A_{1}}^{A_{z}} \partial_{A_{z}}, \\
& \delta_{A_{2}}=\partial_{A_{2}}-N_{A_{2}}^{A_{3}} \partial_{A_{3}}-N_{A_{2}}^{A_{4}} \partial_{A_{4}}-\ldots-N_{A_{2}}^{A_{z-1}} \partial_{A_{z-1}}-N_{A_{2}}^{A_{z}} \partial_{A_{z}} \text {, } \\
& \delta_{A_{z-1}}=\partial_{A_{z-1}}-N_{A_{z-1}}^{A_{z}} \partial_{A_{z}}, \\
& \delta_{A_{z}}=\partial_{A_{z}} \text {, }
\end{aligned}
$$

or, in matrix form, as

$$
\boldsymbol{\delta}_{\bullet}=\widehat{\mathbf{N}}(u) \times \partial_{\bullet},
$$

where

$$
\boldsymbol{\delta}_{\bullet}=\delta_{<\alpha>}=\left(\begin{array}{c}
\delta_{I} \\
\delta_{A_{1}} \\
\delta_{A_{2}} \\
\ldots \\
\delta_{A_{z}}
\end{array}\right)=\left(\begin{array}{c}
\frac{\delta}{\partial x^{I}} \\
\frac{\delta}{\partial y_{(1)}^{A_{1}}} \\
\frac{\delta}{\partial y_{(2)}^{A_{2}}} \\
\ldots \\
\frac{\delta}{\partial y_{(z)}^{A z}}
\end{array}\right), \partial_{\bullet}=\partial_{<\alpha>}=\left(\begin{array}{c}
\partial_{I} \\
\partial_{A_{1}} \\
\partial_{A_{2}} \\
\ldots \\
\partial_{A_{z}}
\end{array}\right)=\left(\begin{array}{c}
\frac{\partial}{\partial x^{I}} \\
\frac{\partial}{\partial y_{(1)}^{A_{1}}} \\
\frac{\partial}{\partial y_{(2)}^{A_{2}}} \\
\ldots \\
\frac{\partial}{\partial y_{(z)}^{A_{z}}}
\end{array}\right) .
$$

and

$$
\widehat{\mathbf{N}}=\left(\begin{array}{ccccc}
1 & -N_{I}^{A_{1}} & -N_{I}^{A_{2}} & \ldots & -N_{I}^{A_{z}} \\
0 & 1 & -N_{A_{1}}^{A_{2}} & \ldots & -N_{A_{1}}^{A_{z}} \\
0 & 0 & 1 & \ldots & -N_{A_{2}}^{A_{z}} \\
\ldots & \ldots & \ldots & \ldots & \ldots \\
0 & 0 & 0 & \ldots & 1
\end{array}\right)
$$

In generalized index form we write the matrix (6) as $\widehat{N}_{<\beta>}^{<\alpha}$, where, for instance, $\widehat{N}_{J}^{I}=\delta_{J}^{I}, \widehat{N}_{B_{1}}^{A_{1}}=\delta_{B_{1}}^{A_{1}}, \ldots, \widehat{N}_{I}^{A_{1}}=-N_{I}^{A_{1}}, \ldots, \widehat{N}_{A_{1}}^{A_{z}}=-N_{A_{1}}^{A_{z}}, \widehat{N}_{A_{2}}^{A_{z}}=-N_{A_{2}}^{A_{z}}, \ldots$.

So in every point $u \in \widetilde{\mathcal{E}}^{<z>}$ we have this invariant decomposition:

$$
T_{u}\left(\widetilde{\mathcal{E}}^{<d>}\right)=N_{0}(u) \oplus N_{1}(u) \oplus \ldots \oplus N_{z-1}(u) \oplus V_{z}(u),
$$

where $\delta_{I} \in N_{0}, \delta_{A_{1}} \in N_{1}, \ldots, \delta_{A_{z-1}} \in N_{z-1}, \partial_{A_{z}} \in V_{z}$. 
We note that for the osculator s-bundle $\left(O s c^{z} \tilde{M}, \pi, \tilde{M}\right)$ there is an additional (we consider the $\mathrm{N}$-adapted variant) s-tangent structure

$$
J: \chi\left(O s c^{z} \tilde{M}\right) \rightarrow \chi\left(O s c^{z} \tilde{M}\right)
$$

defined as

$$
\frac{\delta}{\partial y_{(1)}^{I}}=J\left(\frac{\delta}{\partial x^{I}}\right), \ldots, \frac{\delta}{\partial y_{(z-1)}^{I}}=J\left(\frac{\delta}{\partial y_{(z-2)}^{I}}\right), \frac{\partial}{\partial y_{(z)}^{I}}=J\left(\frac{\delta}{\partial y_{(z-1)}^{I}}\right)
$$

(in this case $I$ - and $A$-indices take the same values and we can not distinguish them), by considering vertical $J$-distributions

$$
N_{0}=N, N_{1}=J\left(N_{0}\right), \ldots, N_{z-1}=J\left(N_{z-2}\right) .
$$

In consequence, for the la-adapted bases on $\left(O s c^{z} \tilde{M}, \pi, \tilde{M}\right)$ there is written this $\mathrm{N}-$ connection matrix:

$$
\mathbf{N}=N_{<I>}^{<J>}=\left(\begin{array}{ccccc}
1 & -N_{(1) I}^{J} & -N_{(2) I}^{J} & \ldots & -N_{(z) I}^{J} \\
0 & 1 & -N_{(1) I}^{J} & \ldots & -N_{(z-1) I}^{J} \\
0 & 0 & 1 & \ldots & -N_{(z-2) I}^{J} \\
\ldots & \ldots & \ldots & \ldots & \ldots \\
0 & 0 & 0 & \ldots & 1
\end{array}\right)
$$

There is a unique distinguished local decomposition of every s-vector $X \in$ $\chi\left(\widetilde{\mathcal{E}}^{<z>}\right)$ on la-base $(12)$ :

$$
X=X^{(H)}+X^{\left(V_{1}\right)}+\ldots+X^{\left(V_{z}\right)},
$$

by using the horizontal, $h$, and verticals, $v_{1}, v_{2}, \ldots, v_{z}$ projections:

$$
X^{(H)}=h X=X^{I} \delta_{I}, X^{\left(V_{1}\right)}=v_{1} X=X^{\left(A_{1}\right)} \delta_{A_{1}}, \ldots, X^{\left(V_{z}\right)}=v_{z} X=X^{\left(A_{z}\right)} \delta_{A_{z}} .
$$

With respect to coordinate transforms (4) the la-bases (12) and ds-vector components (18) are correspondingly transformed as

$$
\frac{\delta}{\partial x^{I}}=\frac{\partial x^{I^{\prime}}}{\partial x^{I}} \frac{\delta}{\partial x^{I^{\prime}}}, \frac{\delta}{\partial y_{(p)}^{A_{p}}}=K_{A_{p}}^{A_{p}^{\prime}} \frac{\delta}{\partial y_{(p)}^{A_{p}^{\prime}}},
$$

and

$$
X^{I^{\prime}}=\frac{\partial x^{I^{\prime}}}{\partial x^{I}} X^{I}, X^{\left(A_{p}^{\prime}\right)}=K_{A_{p}}^{A_{p}^{\prime}} X^{\left(A_{p}^{\prime}\right)}, \forall p=1,2, \ldots z
$$


Under changing of coordinates (3) the local coefficients of a nonlinear connection transform as follows:

$$
Y_{<\alpha>}^{<\alpha^{\prime}>} \widehat{N}_{<\alpha^{\prime}>}^{<\beta^{\prime}>}=\widehat{N}_{<\alpha>}^{<\beta>}\left(K_{<\beta>}^{<\beta^{\prime}>}+Y_{<\beta>}^{<\beta^{\prime}>}\right)
$$

(we can obtain these relations by putting (19) and (6) into (14) where $\widehat{N}_{<\alpha^{\prime}>}^{<\beta^{\prime}>}$ satisfy $\left.\delta_{<\alpha^{\prime}>}=\widehat{N}_{<\alpha^{\prime}>}^{<\beta^{\prime}>} \partial_{<\beta^{\prime}>}\right)$.

For dual la-bases (13) we have these $\mathrm{N}$-connection "prolongations of differentials":

$$
\begin{gathered}
\delta x^{I}=d x^{I}, \\
\delta y^{A_{1}}=d y_{(1)}^{A_{1}}+M_{(1) I}^{A_{1}} d x^{I}, \\
\delta y^{A_{2}}=d y_{(2)}^{A_{2}}+M_{(2) A_{1}}^{A_{2}} d y_{(1)}^{A_{1}}+M_{(2) I}^{A_{2}} d x^{I}, \\
\cdots \cdots \cdots \cdots \cdots \cdots \cdots \cdots \cdots \cdots \cdots \cdots \cdots \cdots \cdots \cdots \cdots \cdots \cdots \cdots \cdots \\
\delta y^{A_{s}}=d y_{(s)}^{A_{s}}+M_{(s) A_{1}}^{A s} d y_{(1)}^{A_{1}}+M_{(s) A_{2}}^{A s} d y_{(2)}^{A_{2}}+\ldots+M_{(z) I}^{A_{z}} d x^{I},
\end{gathered}
$$

where $M_{(\bullet) \bullet}^{\bullet}$ are the dual coefficients of the N-connection which can be expressed explicitly by recurrent formulas through the components of $\mathrm{N}$-connection $N_{<A>}^{<I}$. To do this we shall rewrite formulas (20) in matrix form:

$$
\boldsymbol{\delta}^{\bullet}=\mathbf{d}^{\bullet} \times \mathbf{M}(u)
$$

where

$$
\boldsymbol{\delta}^{\bullet}=\left(\begin{array}{lllll}
\delta x^{I} & \delta y^{A_{1}} & \delta y^{A_{2}} & \ldots & \delta y^{A_{s}}
\end{array}\right), \mathbf{d}^{\bullet}=\left(\begin{array}{lllll}
d x^{I} & d y_{(1)}^{A_{1}} & \delta y_{(2)}^{A_{2}} & \ldots & \delta y_{(s)}^{A_{s}}
\end{array}\right)
$$

and

$$
\mathbf{M}=\left(\begin{array}{ccccc}
1 & M_{(1) I}^{A_{1}} & M_{(2) I}^{A_{2}} & \ldots & M_{(z) I}^{A_{z}} \\
0 & 1 & M_{(2) A_{1}}^{A_{2}} & \ldots & M_{(z) A_{1}}^{A_{z}} \\
0 & 0 & 1 & \ldots & M_{(z) A_{2}}^{A_{z}} \\
\ldots & \ldots & \ldots & \ldots & \ldots \\
0 & 0 & 0 & \ldots & 1
\end{array}\right),
$$

and then, taking into consideration that bases $\partial_{\bullet}\left(\boldsymbol{\delta}_{\bullet}\right)$ and $\mathbf{d}^{\bullet}\left(\boldsymbol{\delta}^{\bullet}\right)$ are mutually dual, to compute the components of matrix $\mathbf{M}$ being $\mathbf{s}$-inverse to matrix $\mathbf{N}$ (see $(17)$ )). We omit these simple but tedious calculus for general dvs-bundles and, for simplicity, we present the basic formulas for osculator sbundle $\left(O s c^{z} \tilde{M}, \pi, \tilde{M}\right)$ when $J$-distribution properties (16) and (17) alleviates the problem. For common type of indices on $\tilde{M}$ and higher order extensions on $O s c^{z} \tilde{M}$ the dual la-base is expressed as

$$
\delta x^{I}=d x^{I},
$$




$$
\begin{gathered}
\delta y_{(1)}^{I}=d y_{(1)}^{I}+M_{(1) J}^{I} d x^{J}, \\
\delta y_{(2)}^{I}=d y_{(2)}^{I}+M_{(1) J}^{I} d y_{(1)}^{J}+M_{(2) J}^{I} d x^{J}, \\
\ldots \ldots \ldots \ldots \ldots \ldots \ldots \ldots \ldots \ldots \ldots \ldots \ldots \ldots \ldots \ldots \ldots \ldots \ldots \ldots \\
\delta y_{(z)}^{I}=d y_{(z)}^{I}+M_{(1) J}^{I} d y_{(s-1)}^{J}+M_{(2) J}^{I} d y_{(z-2)}^{J}+\ldots+M_{(z) J}^{I} d x^{J},
\end{gathered}
$$

with $M$-coefficients computed by recurrent formulas:

$$
\begin{gathered}
M_{(1) J}^{I}=N_{(1) J}^{I}, \\
M_{(2) J}^{I}=N_{(2) J}^{I}+N_{(1) K}^{I} M_{(1) J}^{K}, \\
\cdots \cdots \cdots \cdots \cdots \\
M_{(s) J}^{I}=N_{(s) J}^{I}+N_{(s-1) K}^{I} M_{(1) J}^{K}+\ldots+N_{(2) K}^{I} M_{(z-2) J}^{K}+N_{(1) K}^{I} M_{(z-1) J}^{K} .
\end{gathered}
$$

One holds these transformation law for dual coefficients (21) with respect to coordinate transforms (3) :

$$
\begin{gathered}
M_{(1) J}^{K} Y_{(0,0) K}^{I^{\prime}}=M_{(1) K^{\prime}}^{I^{\prime}} Y_{(0,0) J}^{K^{\prime}}+Y_{(1,0) J}^{I^{\prime}}, \\
M_{(2) J}^{K} Y_{(0,0) K}^{I^{\prime}}=M_{(2) K^{\prime}}^{I^{\prime}} Y_{(0,0) J}^{K^{\prime}}+M_{(1) K^{\prime}}^{I^{\prime}} Y_{(1,0) J}^{K^{\prime}}+Y_{(2,0) J}^{I^{\prime}}, \\
\ldots \ldots \ldots \ldots \ldots \ldots \ldots \ldots \ldots \ldots \ldots \ldots \ldots \ldots \ldots \ldots \ldots \ldots \ldots \ldots \ldots \\
M_{(z) J}^{K} Y_{(0,0) K}^{I^{\prime}}=M_{(z) K^{\prime}}^{I^{\prime}} Y_{(0,0) J}^{K^{\prime}}+M_{(z-1) K^{\prime}}^{I^{\prime}} Y_{(1,0) J}^{K^{\prime}}+\ldots+M_{(1) K^{\prime}}^{I^{\prime}} Y_{(z-1,0) J}^{K^{\prime}}+Y_{(z, 0) J}^{I^{\prime}} .
\end{gathered}
$$

(the proof is a straightforward regroupation of terms after we have put (3) into (21)).

Finally, we note that curvatures of a N-connection in a dvs-bundle $\widetilde{\mathcal{E}}^{<z>}$ can be introduced in a manner similar to that for usual vs-bundles (see (11) by a consequent step by step inclusion of higher dimension anisotropies :

$$
\Omega_{(p)}=\frac{1}{2} \Omega_{(p) \alpha_{p-1} \beta_{p-1}}^{A_{p}} \delta u^{\alpha_{p-1}} \wedge \delta u^{\beta_{p-1}} \otimes \frac{\delta}{\partial y_{(p)}^{A_{p}}}, p=1,2, \ldots, z,
$$

with local coefficients

$$
\begin{gathered}
\Omega_{(p) \beta_{p-1} \gamma_{p-1}}^{A_{p}}=\frac{\delta N_{\beta_{p-1}}^{A_{p}}}{\partial u_{(p-1)}^{\gamma_{p-1}}}-(-)^{\left|\beta_{p-1} \gamma_{p-1}\right|} \frac{\delta N_{\gamma_{p-1}}^{A_{p}}}{\partial u_{(p-1)}^{\beta_{p-1}}}+ \\
N_{\beta_{p-1}}^{D_{p}} \hat{N}_{D_{p} \gamma_{p-1}}^{A_{p}}-(-)^{\left|\beta_{p-1} \gamma_{p-1}\right|} N_{\gamma_{p-1}}^{D_{p}} \hat{N}_{D_{p} \beta_{p-1}}^{A_{p}},
\end{gathered}
$$

where $\hat{N}_{D_{p} \gamma_{p-1}}^{A_{p}}=\frac{\delta N_{\gamma_{p-1}}^{A_{p}}}{\partial y_{(p)}^{D_{p}}}\left(\right.$ we consider $\left.y^{A_{0}} \simeq x^{I}\right)$. 


\section{Geometric Objects in DVS-Bundles}

The geometry of the dvs-bundles is very rich and could have various applications in theoretical and mathematical physics. In this section we shall present the main results from the geometry of total spaces of dvs-bundles.

\subsection{D-tensors and $\mathrm{d}-$-connections in dvs-bundles}

By using adapted bases (12) and (13) one introduces algebra $D T\left(\tilde{\mathcal{E}}^{<z>}\right)$ of distinguished tensor s-fields (ds-fields, ds-tensors, ds-objects) on $\tilde{\mathcal{E}}^{<z>}, \mathcal{T}=$ $\mathcal{T}_{q q_{1} q_{2} \ldots q_{z}}^{p p_{1} p_{2} \ldots p_{z}}$, which is equivalent to the tensor algebra of vs-bundle $\pi_{h v_{1} v_{2} \ldots v_{z}}$ : $H \tilde{\mathcal{E}}^{<z>} \oplus V_{1} \tilde{\mathcal{E}}^{<z>} \oplus V_{2} \tilde{\mathcal{E}}^{<z>} \oplus \ldots \oplus V_{s} \tilde{\mathcal{E}}^{<z>} \rightarrow \tilde{\mathcal{E}}^{<z>}$, hereafter briefly denoted as $\tilde{\mathcal{E}}_{d z}$. An element $Q \in \mathcal{T}_{q q_{1} q_{2} \ldots q_{z}}^{p p_{1} p_{2} \ldots p_{z}}$, , ds-field of type $\left(\begin{array}{ccccc}p & p_{1} & p_{2} & \ldots & p_{z} \\ q & q_{1} & q_{2} & \ldots & q_{z}\end{array}\right)$, can be written in local form as

$$
\begin{gathered}
Q=Q_{J_{1} \ldots J_{q} B_{1} \ldots B_{q_{1}} C_{1} \ldots C_{q_{2}} \ldots F_{1} \ldots F_{q_{s}}}^{I_{1} I_{I_{1}} A_{1} A_{p_{1}} E_{1} \ldots E_{p_{2}} \ldots D_{1} \ldots D_{p_{s}}}(u) \delta_{I_{1}} \otimes \ldots \otimes \delta_{I_{p}} \otimes d^{J_{1}} \otimes \ldots \otimes d^{J_{q}} \otimes \\
\partial_{A_{1}} \otimes \ldots \otimes \partial_{A_{p_{1}}} \otimes \delta^{B_{1}} \delta^{B_{1}} \otimes \ldots \otimes \delta^{B_{q_{1}}} \otimes \partial_{E_{1}} \otimes \ldots \otimes \partial_{E_{p_{2}}} \otimes \delta^{C_{1}} \otimes \ldots \otimes \delta^{C_{q_{2}}} \otimes \ldots \\
\otimes \partial_{D_{1}} \otimes \ldots \otimes \partial_{D_{p_{z}}} \otimes \delta^{F_{1}} \otimes \ldots \otimes \delta^{F_{q z}}
\end{gathered}
$$

In addition to ds-tensors we can introduce ds-objects with various s-group and coordinate transforms adapted to global splitting (15).

A linear distinguished connection, d-connection, in dvs-bundle $\tilde{\mathcal{E}}^{<z>}$ is a linear connection $D$ on $\tilde{\mathcal{E}}^{<z>}$ which preserves by parallelism the horizontal and vertical distributions in $\tilde{\mathcal{E}}^{<z>}$.

By a linear connection of a s-manifold we understand a linear connection in its tangent bundle.

Let denote by $\Xi(\tilde{M})$ and $\Xi\left(\tilde{\mathcal{E}}^{<p>}\right)$, respectively, the modules of vector fields on s-manifold $\tilde{M}$ and vs-bundle $\tilde{\mathcal{E}}^{<p>}$ and by $\mathcal{F}(\tilde{M})$ and $\mathcal{F}\left(\tilde{\mathcal{E}}^{<p>}\right)$, respectively, the s-modules of functions on $\tilde{M}$ and on $\tilde{\mathcal{E}}^{<p>}$.

It is clear that for a given global splitting into horizontal and verticals ssubbundles (15) we can associate operators of horizontal and vertical covariant derivations (h- and $\mathrm{v}$-derivations, denoted respectively as $D^{(h)}$ and $D^{\left(v_{1} v_{2} \ldots v_{z}\right)}$ ) with properties:

$$
D_{X} Y=(X D) Y=D_{h X} Y+D_{v_{1} X} Y+D_{v_{2} X} Y+\ldots+D_{v_{z} X} Y
$$

where

$$
D_{X}^{(h)} Y=D_{h X} Y, \quad D_{X}^{(h)} f=(h X) f
$$

and

$$
D_{X}^{\left(v_{p}\right)} Y=D_{v_{p} X} Y, \quad D_{X}^{\left(v_{p}\right)} f=\left(v_{p} X\right) f,(p=1, \ldots, z)
$$


for every $f \in \mathcal{F}(\tilde{M})$ with decomposition of vectors $X, Y \in \Xi\left(\tilde{\mathcal{E}}^{<z>}\right)$ into horizontal and vertical parts, $X=h X+v_{1} X+\ldots .+v_{z} X$ and $Y=$ $h Y+v_{1} Y+\ldots+v_{z} Y$.

The local coefficients of a d-connection $D$ in $\tilde{\mathcal{E}}^{<z>}$ with respect to the local adapted frame (5) separate into corresponding distinguished groups. We introduce horizontal local coefficients

$$
\begin{gathered}
\left(L_{J K}^{I}, L_{<B>K}^{<A>}\right)=\left(L_{J K}^{I}(u), L_{B_{1} K}^{A_{1}}(u), L_{B_{2} K}^{A_{2}}(u), \ldots, L_{B_{z} K}^{A_{z}}(u)\right) \text { of } D^{(h)} \text { such that } \\
D_{\left(\frac{\delta}{\delta x^{K}}\right)}^{(h)} \frac{\delta}{\delta x^{J}}=L_{J K}^{I}(u) \frac{\delta}{\delta x^{I}}, D_{\left(\frac{\delta}{\delta x^{K}}\right)}^{(h)} \frac{\delta}{\delta y_{(p)}^{B_{p}}}=L_{B_{p} K}^{A_{p}}(u) \frac{\delta}{\delta y_{(p)}^{A_{p}}},(p=1, \ldots, z), \\
D_{\left(\frac{\delta}{\delta x^{k}}\right)}^{(h)} q=\frac{\delta q}{\delta x^{K}},
\end{gathered}
$$

and $p$-vertical local coefficients

$\left(C_{J<C>}^{I}, C_{<B><C>}^{<A>}\right)=\left(C_{J C_{p}}^{I}(u), C_{B_{1} C_{p}}^{A_{1}}(u), C_{B_{2} C_{p}}^{A_{2}}(u), \ldots, C_{B_{z} C_{p}}^{A_{z}}(u)\right)(p=1, \ldots, z)$ such that

$$
D_{\left(\frac{\delta}{\delta y^{C}}\right)}^{\left(v_{p}\right)} \frac{\delta}{\delta x^{J}}=C_{J C_{p}}^{I}(u) \frac{\delta}{\delta x^{I}}, D_{\left(\frac{\delta}{\delta y^{C}}\right)}^{\left(v_{p}\right)} \frac{\delta}{\delta y_{(f)}^{B_{f}}}=C_{B_{f} C_{p}}^{A_{f}} \frac{\delta}{\delta y_{(f)}^{A_{f}}}, D_{\left(\frac{\delta}{\delta y_{p}}\right)}^{\left(v_{p}\right)} q=\frac{\delta q}{\partial y^{C_{p}}},
$$

where $q \in \mathcal{F}\left(\tilde{\mathcal{E}}^{<z>}\right), f=1, \ldots, z$.

The covariant ds-derivation along vector $X=X^{I} \frac{\delta}{\delta x^{I}}+Y^{A_{1}} \frac{\delta}{\delta y^{A_{1}}}+\ldots+Y^{A_{z}} \frac{\delta}{\delta y^{A_{z}}}$ of a ds-tensor field $Q$, for instance, of type $\left(\begin{array}{cc}p & p_{r} \\ q & q_{r}\end{array}\right), 1 \leq r \leq z, \quad$ see $(22)$, can be written as

$$
D_{X} Q=D_{X}^{(h)} Q+D_{X}^{\left(v_{1}\right)} Q+\ldots+D_{X}^{\left(v_{z}\right)} Q
$$

where h-covariant derivative is defined as

$$
D_{X}^{(h)} Q=X^{K} Q_{J B_{r} \mid K}^{I A_{r}} \delta_{I} \otimes \delta_{A_{r}} \otimes d^{I} \otimes \delta^{B_{r}},
$$

with components

$$
Q_{J B_{r} \mid K}^{I A_{r}}=\frac{\delta Q_{J B_{r}}^{I A_{r}}}{\partial x^{K}}+L_{H K}^{I} Q_{J B_{R}}^{H A_{r}}+L_{C_{i} K}^{A_{r}} Q_{J B_{i}}^{I C_{r}}-L_{J K}^{H} Q_{H B_{r}}^{I A_{r}}-L_{B_{r} K}^{C_{r}} Q_{J C_{r}}^{I A_{r}},
$$

and $\mathrm{v}_{p}$-covariant derivatives defined as

$$
D_{X}^{\left(v_{p}\right)} Q=X^{C_{p}} Q_{J B_{r} \perp C_{p}}^{I A_{r}} \delta_{I} \otimes \partial_{A_{r}} \otimes d^{I} \otimes \delta^{B_{r}},
$$

with components

$$
Q_{J B_{r} \perp C_{p}}^{I A_{r}}=\frac{\delta Q_{J B_{R}}^{I A_{r}}}{\partial y^{C_{p}}}+C^{I}{ }_{H C_{p}} Q_{J B_{R}}^{H A_{r}}+C_{F_{r} C_{p}}^{A_{r}} Q_{J B_{R}}^{I F_{r}}-C_{J C_{p}}^{H} Q_{H F_{R}}^{I A_{r}}-C_{B_{r} C_{p}}^{F_{r}} Q_{J F_{R}}^{I A_{r}} .
$$


The above presented formulas show that $D \Gamma=\left(L, \ldots, L_{(p)}, \ldots, C, \ldots, C_{(p)}, \ldots\right)$ are the local coefficients of the d-connection $D$ with respect to the local frame $\left(\frac{\delta}{\delta x^{I}}, \frac{\partial}{\partial y^{a}}\right)$. If a change (3) of local coordinates on $\tilde{\mathcal{E}}^{<z>}$ is performed, by using the law of transformation of local frames (19),we obtain the following transformation laws of the local coefficients of a d-connection:

$$
\begin{gathered}
L_{J^{\prime} M^{\prime}}^{I^{\prime}}=\frac{\partial x^{I^{\prime}}}{\partial x^{I}} \frac{\partial x^{J}}{\partial x^{J^{\prime}}} \frac{\partial x^{M}}{\partial x^{M^{\prime}}} L_{J M}^{I}+\frac{\partial x^{I^{\prime}}}{\partial x^{M}} \frac{\partial^{2} x^{M}}{\partial x^{J^{\prime}} \partial x^{M^{\prime}}}, \\
L_{(f) B_{f}^{\prime} M^{\prime}}^{A_{f}^{\prime}}=K_{A_{f}}^{A_{f}^{\prime}} K_{B_{f}^{\prime}}^{B_{f}} \frac{\partial x^{M}}{\partial x^{M^{\prime}}} L_{(f) B_{f} M}^{A_{f}}+K_{C_{f}}^{A_{f}^{\prime}} \frac{\partial K_{B_{f}^{\prime}}^{C_{f}}}{\partial x^{M^{\prime}}} \\
C_{(p) J^{\prime} C_{p}^{\prime}}^{I^{\prime}}=\frac{\partial x^{I^{\prime}}}{\partial x^{I}} \frac{\partial x^{J}}{\partial x^{J^{\prime}}} K_{C_{p}}^{C_{p}^{\prime}} C_{(p) J C_{p}}^{I}, \ldots, C_{B_{f}^{\prime} C_{p}^{\prime}}^{A^{\prime}}=K_{A_{f}}^{A_{f}^{\prime}} K_{B_{f}^{\prime}}^{B_{f}} K_{C_{p}^{\prime}}^{C_{p}} C_{B_{f} C_{p}}^{A_{f}}, \ldots
\end{gathered}
$$

As in the usual case of tensor calculus on locally isotropic spaces the transformation laws (23) for $\mathrm{d}$-connections differ from those for ds-tensors, which are written (for instance, we consider transformation laws for ds-tensor (22)) as

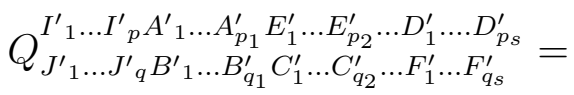

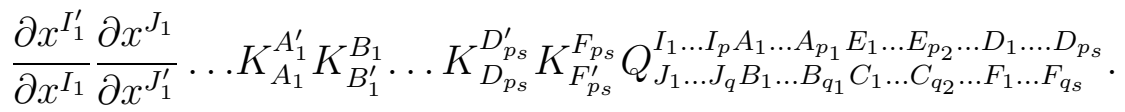

To obtain local formulas on usual higher order anisotropic spaces we have to restrict us with even components of geometric objects by changing, formally, capital indices $(I, J, K, \ldots)$ into $(i, j, k, a, .$.$) and \mathrm{s}$-derivation and $\mathrm{s}-$-commutation rules into those for real number fields on usual manifolds.

\subsection{Torsions and curvatures of $\mathrm{d}-$-connections}

Let $\tilde{\mathcal{E}}^{<z>}$ be a dvs-bundle endowed with N-connection and d-connection structures. The torsion of a $\mathrm{d}$-connection is introduced as

$$
T(X, Y)=[X, D Y\}-[X, Y\}, \quad X, Y \subset \Xi(\tilde{M}) .
$$

One holds the following invariant decomposition (by using $\mathrm{h}-$ and $\mathrm{v}$-projections associated to $\mathrm{N}$ ):

$$
\begin{aligned}
& T(X, Y)=T(h X, h Y)+T\left(h X, v_{1} Y\right)+T\left(v_{1} X, h X\right)+T\left(v_{1} X, v_{1} Y\right)+\ldots \\
& +T\left(v_{p-1} X, v_{p-1} Y\right)+T\left(v_{p-1} X, v_{p} Y\right)+T\left(v_{p} X, v_{p-1} X\right)+T\left(v_{p} X, v_{p} Y\right)+\ldots \\
& \quad+T\left(v_{z-1} X, v_{z-1} Y\right)+T\left(v_{z-1} X, v_{z} Y\right)+T\left(v_{z} X, v_{z-1} X\right)+T\left(v_{z} X, v_{z} Y\right) .
\end{aligned}
$$


Taking into account the skewsupersymmetry of $T$ and the equations

$$
h\left[v_{p} X, v_{p} Y\right\}=0, \ldots, v_{f}\left[v_{p} X, v_{p} Y\right\}=0, f \neq p
$$

we can verify that the torsion of a $\mathrm{d}$-connection is completely determined by the following ds-tensor fields:

$$
\begin{gathered}
h T(h X, h Y)=\left[X\left(D^{(h)} h\right) Y\right\}-h[h X, h Y\}, \ldots, \\
v_{p} T(h X, h Y)=-v_{p}[h X, h Y\}, \ldots, \\
h T\left(h X, v_{p} Y\right)=-D_{Y}^{\left(v_{p}\right)} h X-h\left[h X, v_{p} Y\right\}, \ldots, \\
v_{p} T\left(h X, v_{p} Y\right)=D_{X}^{(h)} v_{p} Y-v_{p}\left[h X, v_{p} Y\right\}, \ldots, \\
v_{f} T\left(v_{f} X, v_{f} Y\right)=\left[X\left(D^{\left(v_{f}\right)} v_{f}\right) Y\right\}-v_{f}\left[v_{f} X, v_{f} Y\right\}, \ldots, \\
v_{p} T\left(v_{f} X, v_{f} Y\right)=-v_{p}\left[v_{f} X, v_{f} Y\right\}, \ldots, \\
v_{f} T\left(v_{f} X, v_{p} Y\right)=-D_{Y}^{\left(v_{p}\right)} v_{f} X-v_{f}\left[v_{f} X, v_{p} Y\right\}, \ldots, \\
v_{p} T\left(v_{f} X, v_{p} Y\right)=D_{X}^{\left(v_{f}\right)} v_{p} Y-v_{p}\left[v_{f} X, v_{p} Y\right\}, \ldots, f<p, \\
v_{z-1} T\left(v_{z-1} X, v_{x-1} Y\right)=\left[X\left(D^{\left(v_{z-1}\right)} v_{z-1}\right) Y\right\}-v_{z-1}\left[v_{z-1} X, v_{z-1} Y\right\}, \ldots, \\
v_{z} T\left(v_{z-1} X, v_{z-1} Y\right)=-v_{z}\left[v_{z-1} X, v_{z-1} Y\right\} \\
v_{z-1} T\left(v_{z-1} X, v_{z} Y\right)=-D_{Y}^{\left(v_{z}\right)} v_{z-1} X-v_{z-1}\left[v_{z-1} X, v_{z} Y\right\}, \ldots, \\
v_{z} T\left(v_{z-1} X, v_{z} Y\right)=D_{X}^{\left(v_{z-1}\right)} v_{z} Y-v_{z}\left[v_{z-1} X, v_{z} Y\right\}
\end{gathered}
$$

where $X, Y \in \Xi\left(\tilde{\mathcal{E}}^{<z>}\right)$. In order to get the local form of the ds-tensor fields which determine the torsion of $\mathrm{d}$-connection $D \Gamma$ (the torsions of $D \Gamma$ ) we use equations

$$
\left[\frac{\delta}{\partial x^{J}}, \frac{\delta}{\partial x^{K}}\right\}=R_{J K}^{<A>} \frac{\delta}{\partial y^{<A>}},\left[\frac{\delta}{\partial x^{J}}, \frac{\delta}{\partial y^{<B>}}\right\}=\frac{\delta N_{J}^{<A>}}{\partial y^{<B>}} \frac{\delta}{\partial y^{<A>}},
$$

where

$$
R_{J K}^{<A>}=\frac{\delta N_{J}^{<A>}}{\partial x^{K}}-(-)^{|K J|} \frac{\delta N_{K}^{<A>}}{\partial x^{J}},
$$

and introduce notations

$$
\begin{gathered}
h T\left(\frac{\delta}{\delta x^{K}}, \frac{\delta}{\delta x^{J}}\right)=T_{J K}^{I} \frac{\delta}{\delta x^{I}}, \\
v_{1} T\left(\frac{\delta}{\delta x^{K}}, \frac{\delta}{\delta x^{J}}\right)=\tilde{T}_{K J}^{A_{1}} \frac{\delta}{\partial y^{A_{1}}},
\end{gathered}
$$




$$
\begin{gathered}
h T\left(\frac{\delta}{\partial y^{<A>}}, \frac{\delta}{\partial x^{J}}\right)=\tilde{P}_{J<A>}^{I} \frac{\delta}{\delta x^{I}}, \ldots, \\
v_{p} T\left(\frac{\delta}{\partial y^{B_{p}}}, \frac{\delta}{\delta x^{J}}\right)=P_{J B_{p}}^{<A>} \frac{\delta}{\partial y^{<A>}}, \ldots, \\
v_{p} T\left(\frac{\delta}{\partial y^{C_{p}}}, \frac{\delta}{\partial y^{B_{f}}}\right)=S_{B_{f} C_{p}}^{<A>} \frac{\delta}{\partial y^{<A>}} .
\end{gathered}
$$

Now we can compute the local components of the torsions, introduced in (24), with respect to a la-frame of a d-connection

$$
\begin{aligned}
& D \Gamma=\left(L, \ldots, L_{(p)}, \ldots, C, \ldots, C_{(p)}, \ldots\right) \text { : } \\
& T_{J K}^{I}=L_{J K}^{I}-(-)^{|J K|} L_{K J}^{I}, \\
& \tilde{T}_{J K}^{<A>}=R_{J K}^{<A>}, \tilde{P}_{J<B>}^{I}=C_{J<B>}^{I}, \\
& P_{J<B>}^{<A>}=\frac{\delta N_{J}^{<A>}}{\partial y^{<B>}}-L_{<A>J}^{<,} \\
& S^{<A>}<B><C>=C^{<A>}<B><C>-(-)^{|<B><C>|} C^{<A>}<C><B>.
\end{aligned}
$$

The even and odd components of torsions (25) can be specified in explicit form by using decompositions of indices into even and odd parts $(I=(i, \hat{i}), J=$ $(j, \hat{j}), .$.$) , for instance,$

$$
\begin{gathered}
T_{j k}^{i}=L_{j k}^{i}-L_{k j}^{i}, \quad T_{j \hat{k}}^{i}=L_{j \hat{k}}^{i}+L_{\hat{k} j}^{i}, \\
T_{j k}^{\hat{i}}=L_{j k}^{\hat{i}}-L_{k j}^{\hat{i}}, \ldots,
\end{gathered}
$$

and so on (see [75, 76]).

Another important characteristic of a $\mathrm{d}-$-connection $D \Gamma$ is its curvature:

$$
R(X, Y) Z=D_{[X} D_{Y\}}-D_{[X, Y\}} Z
$$

where $X, Y, Z \in \Xi\left(\tilde{E}^{<z>}\right)$. From the properties of h- and v-projections it follows that

$$
v_{p} R(X, Y) h Z=0, \ldots, h R(X, Y) v_{p} Z=0, v_{f} R(X, Y) v_{p} Z=0, f \neq p
$$

and

$$
R(X, Y) Z=h R(X, Y) h Z+v_{1} R(X, Y) v_{1} Z+\ldots+v_{z} R(X, Y) v_{z} Z
$$

where $X, Y, Z \in \Xi\left(\tilde{E}^{<z>}\right)$. Taking into account properties (26) and the equations

$$
R(X, Y)=-(-)^{|X Y|} R(Y, X)
$$


we prove that the curvature of a d-connection $D$ in the total space of a vs-bundle $\tilde{E}^{<z>}$ is completely determined by the following ds-tensor fields:

$$
\begin{gathered}
R(h X, h Y) h Z=\left(D_{[X}^{(h)} D_{Y\}}^{(h)}-D_{[h X, h Y\}}^{(h)}\right. \\
\left.-D_{[h X, h Y\}}^{\left(v_{1}\right)}-\ldots D_{[h X, h Y\}}^{\left(v_{z-1}\right)}-D_{[h X, h Y\}}^{\left(v_{z}\right)}\right) h Z \\
R(h X, h Y) v_{p} Z=\left(D_{[X}^{(h)} D_{Y\}}^{(h)}-D_{[h X, h Y\}}^{(h)}-\right. \\
\left.\left.D_{[h X, h Y\}}^{\left(v_{1}\right)} v_{p} Z-\ldots-D_{[h X, h Y\}}^{\left(v_{p-1}\right)}\right) v_{p} Z-D_{[h X, h Y\}}^{\left(v_{p}\right)}\right) v_{p} Z \\
R\left(v_{p} X, h Y\right) h Z=\left(D_{[X}^{\left(v_{p}\right)} D_{Y\}}^{(h)}-D_{\left[v_{p} X, h Y\right\}}^{(h)}-\right. \\
\left.D_{\left[v_{p} X, h Y\right\}}^{\left(v_{1}\right)} h Z-\ldots-D_{\left[v_{p} X, h Y\right\}}^{\left(v_{p-1}\right)}-D_{\left[v_{p} X, h Y\right\}}^{\left(v_{p}\right)}\right) h Z \\
R\left(v_{f} X, h Y\right) v_{p} Z=\left(D_{[X}^{\left(v_{f}\right)} D_{Y\}}^{(h)}-D_{\left[v_{f} X, h Y\right\}}^{(h)}-\right. \\
\left.\left.\left.D_{\left[v_{f} X, h Y\right\}}^{\left(v_{1}\right)}\right) v_{1} Z-\ldots-D_{\left[v_{f} X, h Y\right\}}^{\left(v_{p-1}\right)}\right) v_{p-1} Z-D_{\left[v_{f} X, h Y\right\}}^{\left(v_{p}\right)}\right) v_{p} Z \\
R\left(v_{f} X, v_{p} Y\right) h Z=\left(D_{[X}^{\left(v_{f}\right)} D_{Y\}}^{\left(v_{p}\right)}-D_{\left[v_{f} X, v_{p} Y\right\}}^{\left(v_{1}\right)} h Z-\ldots\right. \\
-D_{\left[v_{f} X, v_{p} Y\right\}}^{\left(v_{z-1}\right)}-D_{\left[v_{f} X, v_{p} Y\right\}}^{\left(v_{z}\right)} h Z, \\
R\left(v_{f} X, v_{q} Y\right) v_{p} Z=\left(D_{[X}^{\left(v_{f}\right)} D_{Y\}}^{\left(v_{q}\right)}-D_{\left[v_{f} X, v_{q} Y\right\}}^{\left(v_{1}\right)}\right) v_{1} Z-\ldots \\
\left.\left.-D_{\left[v_{f} X, v_{q} Y\right\}}^{\left(v_{p-1}\right)}\right) v_{p-1} Z-D_{\left[v_{f} X, v_{q} Y\right\}}^{\left(v_{p}\right)}\right) v_{p} Z
\end{gathered}
$$

where

$$
\begin{gathered}
D_{[X}^{(h)} D_{Y\}}^{(h)}=D_{X}^{(h)} D_{Y}^{(h)}-(-)^{|X Y|} D_{Y}^{(h)} D_{X}^{(h)}, \\
D_{[X}^{(h)} D_{Y\}}^{\left(v_{p}\right)}=D_{X}^{(h)} D_{Y}^{\left(v_{p}\right)}-(-)^{\left|X v_{p} Y\right|} D_{Y}^{\left(v_{p}\right)} D_{X}^{(h)}, \\
D_{[X}^{\left(v_{p}\right)} D_{Y\}}^{(h)}=D_{X}^{\left(v_{p}\right)} D_{Y}^{(h)}-(-)^{\left|v_{p} X Y\right|} D_{Y}^{(h)} D_{X}^{\left(v_{p}\right)}, \\
D_{[X}^{\left(v_{f}\right)} D_{Y\}}^{\left(v_{p}\right)}=D_{X}^{\left(v_{f}\right)} D_{Y}^{\left(v_{p}\right)}-(-)^{\left|v_{f} X v_{p} Y\right|} D_{Y}^{\left(v_{p}\right)} D_{X}^{\left(v_{f}\right)} .
\end{gathered}
$$

The local components of the ds-tensor fields (27) are introduced as follows:

$$
\begin{gathered}
R\left(\delta_{K}, \delta_{J}\right) \delta_{H}=R_{H J K}^{I} \delta_{I}, R\left(\delta_{K}, \delta_{J}\right) \delta_{<B>}=R_{<B>. J K}^{<A>} \delta_{<A>}, \\
R\left(\delta_{<C>}, \delta_{K}\right) \delta_{J}=P_{J K<C>}^{I} \delta_{I}, R\left(\delta_{<C>}, \delta_{K}\right) \delta_{<B>}=P_{<B>. K<C>}^{\cdot<A>} \delta_{<A>}, \\
R\left(\delta_{<C>}, \delta_{<B>}\right) \delta_{J}=S_{J \cdot<B><C>}^{I} \delta_{I}, R\left(\delta_{<D>}, \delta_{<C>}\right) \delta_{<B>}=S_{<B>.<C><D>}^{<<>} \delta_{<A>} .
\end{gathered}
$$


Putting the components of a d-connection $D \Gamma=\left(L, \ldots, L_{(p)}, \ldots, C, \ldots, C_{(p)}, \ldots\right)$ in (28), by a direct computation, we obtain these locally adapted components of the curvature (curvatures):

$$
\begin{aligned}
& R_{H}^{I}{ }_{J K}=\delta_{K} L_{H J}^{I}-(-)^{|K J|} \delta_{J} L_{H K}^{I}+ \\
& L_{H J}^{M} L_{M K}^{I}-(-)^{|K J|} L^{M}{ }_{H K} L^{I}{ }_{M J}+C_{H<A>}^{I} R^{<A>}{ }_{J K}, \\
& R_{<B>\cdot J K}^{<A>}=\delta_{K} L^{<A>}{ }_{<B>J}-(-)^{|K J|} \delta_{J} L^{<A>}{ }_{<B>K}+ \\
& L^{<C>}{ }_{<B>J} L^{<A>}{ }_{<C>K}-(-)^{|K J|} L^{<C>}{ }_{<B>K}+C^{<A>}{ }_{<B><C>} R^{<C>}{ }_{J K}, \\
& P_{J \cdot K<A>}^{\cdot I}=\delta_{<A>} L_{J K}^{I}-C_{J<A>\mid K}^{I}+C_{J<B>}^{I} P_{K<A>}^{<>} \text {, }
\end{aligned}
$$

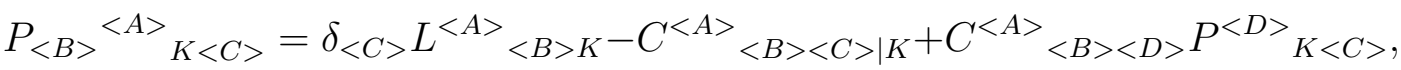

$$
\begin{aligned}
& S_{J \cdot<B><C>}^{I I}=\delta_{<C>} C^{I}{ }_{J<B>}-(-)^{|<B><C>|} \delta_{<B>} C^{I}{ }_{J<C>}+ \\
& C^{<H>}{ }_{J<B>} C^{I}{ }_{<H><C>}-(-)^{|<B><C>|} C^{<H>}{ }_{J<C>} C^{I}{ }_{<H><B>},
\end{aligned}
$$

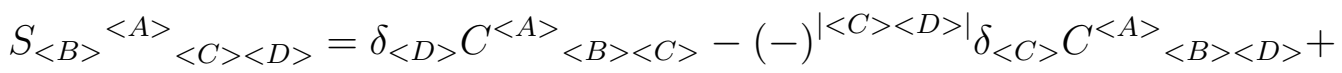

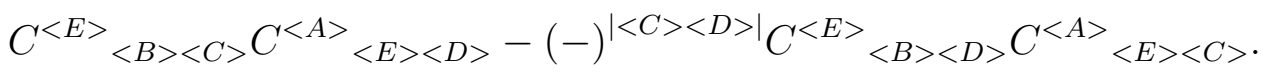

Even and odd components of curvatures (29) can be written out by splitting indices into even and odd parts, for instance,

$$
\begin{gathered}
R_{h}{ }_{j k}=\delta_{k} L^{i}{ }_{h j}-\delta_{j} L_{h k}^{i}+L^{m}{ }_{h j} L^{i}{ }_{m k}-L^{m}{ }_{h k} L^{i}{ }_{m j}+C^{i}{ }_{h<a>} R^{<a>}{ }_{j k}, \\
R_{h}{ }_{j \hat{k}}=\delta_{\hat{k}} L_{h j}^{i}+\delta_{j} L_{h \hat{k}}^{i}+L^{m}{ }_{h j} L^{i}{ }_{m \hat{k}}+L^{m}{ }_{h \hat{k}} L^{i}{ }_{m j}+C_{h<a>}^{i} R^{<a>}{ }_{j \hat{k}} \quad, \ldots
\end{gathered}
$$

(we omit formulas for the even-odd components of curvatures because we shall not use them in this work).

\subsection{Bianchi and Ricci identities}

The torsions and curvatures of every linear connection $D$ on a vs-bundle $\tilde{\mathcal{E}}^{<z>}$ satisfy the following generalized Bianchi identities:

$$
\begin{gathered}
\sum_{S C}\left[\left(D_{X} T\right)(Y, Z)-R(X, Y) Z+T(T(X, Y), Z)\right]=0, \\
\sum_{S C}\left[\left(D_{X} R\right)(U, Y, Z)+R(T(X, Y) Z) U\right]=0,
\end{gathered}
$$

where $\sum_{S C}$ means the respective supersymmretric cyclic sum over $X, Y, Z$ and $U$. If $D$ is a d-connection, then by using (26) and

$$
v_{p}\left(D_{X} R\right)(U, Y, h Z)=0, h\left(D_{X} R\left(U, Y, v_{p} Z\right)=0, v_{f}\left(D_{X} R\right)\left(U, Y, v_{p} Z\right)=0,\right.
$$


the identities (30) become

$$
\begin{gathered}
\sum_{S C}\left[h\left(D_{X} T\right)(Y, Z)-h R(X, Y) Z+h T(h T(X, Y), Z)+\right. \\
\left.h T\left(v_{1} T(X, Y), Z\right)+\ldots+h T\left(v_{z} T(X, Y), Z\right)\right]=0, \\
\sum_{S C}\left[v_{f}\left(D_{X} T\right)(Y, Z)-v_{f} R(X, Y) Z+\right. \\
\left.v_{f} T(h T(X, Y), Z)+\sum_{p \geq f} v_{f} T\left(v_{p} T(X, Y), Z\right)\right]=0, \\
\sum_{S C}\left[h\left(D_{X} R\right)(U, Y, Z)+h R(h T(X, Y), Z) U+\right. \\
\left.h R\left(v_{1} T(X, Y), Z\right) U+\ldots+h R\left(v_{z} T(X, Y), Z\right) U\right]=0, \\
\sum_{S C}\left[v_{f}\left(D_{X} R\right)(U, Y, Z)+v_{f} R(h T(X, Y), Z) U+\right. \\
\left.\sum_{p \geq f} v_{f} R\left(v_{p} T(X, Y), Z\right) U\right]=0 .
\end{gathered}
$$

In order to get the component form of these identities we insert correspondingly in (31) these values of triples $(X, Y, Z),\left(=\left(\delta_{J}, \delta_{K}, \delta_{L}\right)\right.$, or $\left.\left(\delta_{<D>}, \delta_{<C>}, \delta_{<B>}\right)\right)$, and put successively $U=\delta_{H}$ and $U=\delta_{<A>}$. Taking into account (24),(25) and (27),(28) we obtain:

$$
\begin{aligned}
& \sum_{S C[L, K, J\}}\left[T_{J K \mid H}^{I}+T_{J K}^{M} T_{H M}^{J}+R^{<A>}{ }_{J K} C_{H<A>}^{I}-R_{J}^{I}{ }_{K H}\right]=0, \\
& \sum_{S C[L, K, J\}}\left[R^{<A>}{ }_{J K \mid H}+T_{J K}^{M} R_{H M}^{<A>}+R_{J K}^{<B>} P_{H<B>}^{<A>}\right]=0, \\
& C^{I}{ }_{J<B>\mid K}-(-)^{|J K|} C_{K<B>\mid J}^{I}-T_{J K \mid<B>}^{I}+C_{J<B>}^{M} T_{K M}^{I}- \\
& (-)^{|J K|} C_{K<B>}^{M} T_{J M}^{I}+T_{J K}^{M} C_{M<B>}^{I}+P_{J<B>}^{<D>} C_{K<D>}^{I} \\
& -(-)^{|K J|} P_{K<B>}^{<D>} C_{J<D>}^{I}+P_{J}^{I}{ }_{K<B>}-(-)^{|K J|} P_{K}^{I}{ }_{J<B>}=0, \\
& P_{J<B>\mid K}^{<A>}-(-)^{|K J|} P_{K<B>\mid J}^{<A>}-R_{J K \perp<B>}^{<A>}+C_{J<B>}^{M} R_{K M}^{<A>} \\
& (-)^{|K J|} C_{K<B>}^{M} R_{J M}^{<A>}+T_{J K}^{M} P_{M<B>}^{<A>}+P_{J<B>}^{<D>} P_{K<D>}^{<A>} \\
& (-)^{|K J|} P_{K<B>}^{<D>} P_{J<D>}^{<A>}-R_{J K}^{<D>} S_{B<D>}+R_{<B>\cdot J K}^{<A>}=0, \\
& C_{J<B>\perp<C>}^{I}-(-)^{|<B><C>|} C^{I}{ }_{J<C>\perp<B>}+C^{M}{ }_{J<C>} C_{M<B>}^{I}- \\
& (-)^{|<B><C>|} C_{J<B>}^{M} C^{I}{ }_{M<C>}+S^{<D>}{ }_{<B><C>} C_{J<D>}^{I}-S_{J \cdot<B><C>}^{\cdot I}=0, \\
& P_{J<B>\perp<C>}^{<A>}-(-)^{|<B><C>|} P_{J<C>\perp<B>}^{<A>}
\end{aligned}
$$




$$
\begin{aligned}
& S^{<A>}<B><C>\mid J+C_{J<C>}^{M} P_{M<B>}^{<A>} \\
& (-)^{|<B><C>|} C_{J<B>}^{M} P_{M<C>}^{<A>}+P_{J<B>}^{<D>} S_{<C><D>}- \\
& (-)^{|<C><B>|} P^{<D>}{ }_{J<C>} S^{<A>}{ }_{<B<D>}+S^{<D>}{ }_{<B><C>} P_{J<D>}^{<A>} \\
& P_{<B>}{ }_{J<C>}-(-)^{|<C><B>|} P_{<C>}{ }_{J<B>}=0, \\
& \sum_{S C[<B>,<C>,<D>\}}\left[S^{<A>}<B><C>\perp<D>+\right. \\
& \left.S^{<F>}<B><C>S^{<A>}<D><F>-S_{<B>}<A><C><D>\right]=0, \\
& \sum_{S C[H, J, L\}}\left[R_{K}^{I}{ }_{H J \mid L}-T_{H J}^{M} R_{K}^{I}{ }_{L M}-R_{H J}^{<A>} P_{K \cdot L<A>}^{\cdot I}\right]=0, \\
& \sum_{S C[H, J, L\}}\left[R_{<D>\cdot H J \mid L}^{<A>}-T_{H J}^{M} R_{<D>\cdot L M}^{<A>}-R_{H J}^{<C>} P_{<D>}{ }_{L<C>}^{<A>}\right]=0, \\
& P_{K \cdot J<D>\mid L}^{\cdot I}-(-)^{|L J|} P_{K \cdot L<D>\mid J}^{\cdot I}+R_{K}^{I}{ }_{L J \perp<D>}+C_{L<D>}^{M} R_{K}{ }^{I}{ }^{\prime} M^{-} \\
& (-)^{|L J|} C_{J<D>}^{M} R_{K}^{I}{ }_{L M}-T_{J L}^{M} P_{K \cdot M<D>}^{\cdot I}+ \\
& P^{<A>}{ }_{L<D>} P_{K \cdot J<A>}^{\cdot I}-(-)^{|L J|} P_{J<D>}^{<A>} P_{K \cdot L<A>}^{\cdot I}-R^{<A>}{ }_{L} S_{K \cdot<A><D>}^{\cdot I}=0, \\
& P_{<C>}{ }_{J<D>\mid L}-(-)^{|L J|} P_{<C>}{ }_{L<D>\mid J}+R_{<C>L J \mid<D>}^{<A>}+ \\
& C_{L<D>}^{M} R_{<C>}{ }_{J M}-(-)^{|L J|} C_{J<D>}^{M} R_{<C>}{ }_{L M}- \\
& T_{J L}^{M} P_{<C>}{ }_{M<D>}+P_{L<D>}^{<F>} P_{<C>}{ }_{J<F>}- \\
& (-)^{|L J|} P^{<F>}{ }_{J<D>} P_{<C>}{ }_{L<F>}-R^{<F>}{ }_{J L} S_{<C>}{ }_{F<D>}=0, \\
& P_{K \cdot J<D>\perp<C>}^{\cdot I}-(-)^{|<C><D>|} P_{K \cdot J<C>\perp<D>}^{\cdot I}+S_{K}^{I}<D><C>\mid J^{+} \\
& C_{J<D>}^{M} P_{K \cdot M<C>}^{\cdot I}-(-)^{|<C><D>|} C_{J<C>}^{M} P_{K \cdot M<D>}^{\cdot I}+ \\
& P_{J<C>}^{<A>} S_{K \cdot<D><A>}^{\cdot I}-(-)^{|<C><D>|} P_{J<D>}^{<A>} S_{K \cdot<C><A>}^{\cdot I}+ \\
& S^{<A>}<C><D>P_{K \cdot J<A>}^{\cdot I}=0, \\
& P_{<B>}{ }^{<A>}{ }_{J<D>\perp<C>}-(-)^{|<C><D>|} P_{<B>}{ }_{J<C>\perp<D>}+S_{<B>}{ }^{<A>}<C><D>\mid J+ \\
& C_{J<D>}^{M} P_{<B>}<A>{ }_{M<C>}-(-)^{|<C><D>|} C_{J<C>}^{M} P_{<B>}{ }_{M<D>}+
\end{aligned}
$$

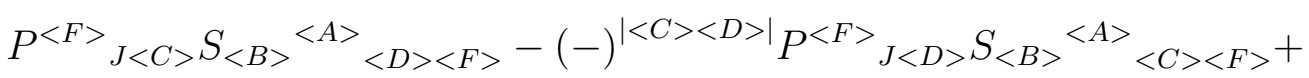

$$
\begin{aligned}
& S^{<F>}{ }_{<C><D>} P_{<B>}{ }_{J<F>}=0, \\
& \sum_{S C[<B>,<C>,<D>\}}\left[S_{K .<B><C>\perp<D>}^{. I}-S_{.<B><C>}^{<A>} S_{K .<D><A>}^{I}\right]=0,
\end{aligned}
$$




$$
\sum_{S C[<B>,<C>,<D>\}}\left[S_{<F>.<B><C>\perp<D>}^{<A>}-S_{.<B><C>}^{<E>} S_{<F>.<E><A>}^{\cdot<A>}\right]=0,
$$

where $\sum_{S C[<B>,<C>,<D>\}} i$ is the supersymmetric cyclic sum over indices $\langle B\rangle$, $<C>,<D>$.

As a consequence of a corresponding arrangement of (27) we obtain the Ricci identities (for simplicity we establish them only for ds-vector fields, although they may be written for every ds-tensor field):

$$
\begin{gathered}
D_{[X}^{(h)} D_{Y\}}^{(h)} h Z=R(h X, h Y) h Z+D_{[h X, h Y\}}^{(h)} h Z+\sum_{f=1}^{z} D_{[h X, h Y\}}^{\left(v_{f}\right)} h Z, \\
D_{[X}^{\left(v_{p}\right)} D_{Y\}}^{(h)} h Z=R\left(v_{p} X, h Y\right) h Z+D_{\left[v_{p} X, h Y\right\}}^{(h)} h Z+\sum_{f=1}^{z} D_{\left[v_{p} X, h Y\right\}}^{\left(v_{f}\right)} h Z, \\
D_{[X}^{\left(v_{p}\right)} D_{Y\}}^{\left(v_{P}\right)}=R\left(v_{p} X, v_{p} Y\right) h Z+\sum_{f=1}^{z} D_{\left[v_{p} X, v_{p} Y\right\}}^{\left(v_{f}\right)} h Z
\end{gathered}
$$

and

$$
\begin{gathered}
D_{[X}^{(h)} D_{Y\}}^{(h)} v_{p} Z=R(h X, h Y) v_{p} Z+D_{[h X, h Y\}}^{(h)} v_{p} Z+\sum_{f=1}^{z} D_{[h X, h Y\}}^{\left(v_{f}\right)} v_{p} Z, \\
D_{[X}^{\left(v_{f}\right)} D_{Y\}}^{(h)} v_{p} Z=R\left(v_{f} X, h Y\right) v_{p} Z+\sum_{q=1}^{z} D_{\left[v_{f} X, h Y\right\}}^{\left(v_{q}\right)} v_{p} Z+\sum_{q=1}^{z} D_{\left[v_{f} X, h Y\right\}}^{\left(v_{q}\right)} v_{p} Z, \\
D_{[X}^{\left(v_{q}\right)} D_{Y\}}^{\left(v_{f}\right)} v_{p} Z=R\left(v_{q} X, v_{f} Y\right) v_{p} Z+\sum_{s=1}^{z} D_{\left[v_{f} X, v_{f} Y\right\}}^{\left(v_{s}\right)} v_{p} Z .
\end{gathered}
$$

Putting $X=X^{I}(u) \frac{\delta}{\delta x^{I}}+X^{<A>}(u) \frac{\delta}{\partial y^{<A>}}$ and taking into account the local form of the h- and v-covariant s-derivatives and $(24),(25),(27),(28)$ we can express respectively identities (32) and (33) in this form:

$$
\begin{aligned}
& X^{<A>}{ }_{|K| L}-(-)^{|K L|} X^{<A>}{ }_{|L| K}= \\
& R_{<B>}{ }^{<A>}{ }_{K L} X^{<B>}-T^{H}{ }_{K L} X^{<A>}{ }_{\mid H}-R^{<B>}{ }_{K L} X^{<A>}{ }_{\perp<B>}, \\
& X_{\mid K \perp<D>-(-)^{|K<D>|}}^{I} X_{\perp<D>\mid K}^{I}= \\
& P_{H \cdot K<D>}^{\cdot I} X^{H}-C_{K<D>}^{H} X_{\mid H}^{I}-P_{K<D>}^{<A>} X_{\perp<A>,}^{I}, \\
& X_{\perp<B>\perp<C>}^{I}-(-)^{|<B><C>|} X_{\perp<C>\perp<B>}^{I}= \\
& S_{H \cdot<B><C>}^{\cdot I} X^{H}-S^{<A>}{ }_{<B<C C>} X_{\perp<A>}^{I}
\end{aligned}
$$

and

$$
X^{<A>}{ }_{|K| L}-(-)^{|K L|} X^{<A>}{ }_{|L| K}=
$$




$$
\begin{aligned}
& R_{<B>}{ }^{<A>}{ }_{K L} X^{<B>}-T^{H}{ }_{K L} X^{<A>}{ }_{\mid H}-R^{<B>}{ }_{K L} X^{<A>}{ }_{\perp<B>}, \\
& X^{<A>} \mid K \perp<B>-(-)^{|<B>K|} X^{<A>}{ }_{\perp<B>\mid K}= \\
& P_{<B>}{ }^{<A>}{ }_{K C} X^{C}-C_{K<B>}^{H} X^{<A>}{ }_{\mid H}-P_{K<B>}^{<D>} X_{\perp<D>}^{<A>}, \\
& X_{\perp<B>\perp<C>}^{<A>}-(-)^{|<C><B>|} X_{\perp<C>\perp<B>}^{<A>}= \\
& S_{<D>}{ }^{<A>}{ }_{<B><C>} X^{<D>}-S^{<D>}{ }_{<B><C>} X^{<A>}{ }_{\perp<D>} .
\end{aligned}
$$

We note that the above presented formulas [110 generalize for higher order anisotropy the similar ones for locally anisotropic superspaces [106].

\subsection{Cartan structure equations in dvs-bundles}

Let consider a ds-tensor field on $\tilde{\mathcal{E}}^{<z>}$ :

$$
t=t_{<A>}^{I} \delta_{I} \otimes \delta^{<A>} .
$$

The d-connection 1-forms $\omega_{J}^{I}$ and $\tilde{\omega}_{<B>}^{<A>}$ are introduced as

$$
D t=\left(D t_{<A>}^{I}\right) \delta_{I} \otimes \delta^{<A>}
$$

with

$$
D t_{<A>}^{I}=d t_{<A>}^{I}+\omega_{J}^{I} t_{<A>}^{J}-\tilde{\omega}_{<A>}^{<B>} t_{<B>}^{I}=t_{<A>\mid J}^{I} d x^{J}+t_{<A>\perp<B>}^{I} \delta y^{<B>} .
$$

For the d-connection 1-forms of a d-connection $D$ on $\tilde{\mathcal{E}}^{<z>}$ defined by $\omega_{J}^{I}$ and $\tilde{\omega}_{<B>}^{<A}>$ one holds the following structure equations:

$$
\begin{gathered}
d\left(d^{I}\right)-d^{H} \wedge \omega_{H}^{I}=-\Omega, d\left(\delta^{<A>}\right)-\delta^{<B>} \wedge \tilde{\omega}_{<B>}^{<A>}=-\tilde{\Omega}^{<A>}, \\
d \omega_{J}^{I}-\omega_{J}^{H} \wedge \omega_{H}^{I}=-\Omega_{J}^{I}, d \tilde{\omega}_{<B>}^{<A>}-\tilde{\omega}_{<B>}^{<C>} \wedge \tilde{\omega}_{<C>}^{<A>}=-\tilde{\Omega}_{<B>}^{<A>},
\end{gathered}
$$

in which the torsion 2-forms $\Omega^{I}$ and $\tilde{\Omega}^{<A>}$ are given respectively by formulas:

$$
\begin{gathered}
\Omega^{I}=\frac{1}{2} T_{J K}^{I} d^{J} \wedge d^{K}+\frac{1}{2} C^{I}{ }_{J<C>} d^{J} \wedge \delta^{<C>}, \\
\tilde{\Omega}^{<A>}=\frac{1}{2} R_{J K}^{<A>} d^{J} \wedge d^{K}+\frac{1}{2} P^{<A>}{ }_{J<C>} d^{J} \wedge \delta^{<C>}+\frac{1}{2} S^{<A>}{ }_{<><C>} \delta^{<B>} \wedge \delta^{<C>},
\end{gathered}
$$

and

$$
\begin{aligned}
& \Omega_{J}^{I}=\frac{1}{2} R_{J}^{I}{ }_{K H} d^{K} \wedge d^{H}+\frac{1}{2} P_{J \cdot K<C>}^{\cdot I} d^{K} \wedge \delta^{<C>}+\frac{1}{2} S_{J \cdot K<C>}^{\cdot I} \delta^{<B>} \wedge \delta^{<C>}, \\
& \tilde{\Omega}_{<B>}^{<A>}=\frac{1}{2} R_{<B>\cdot K H}^{<A>} d^{K} \wedge d^{H}+ \\
& \frac{1}{2} P_{<B>}{ }_{K<C>} d^{K} \wedge \delta^{<C>}+\frac{1}{2} S_{<B>}{ }_{<A>}{ }_{<C>D>} \delta^{<C>} \wedge \delta^{<D>} .
\end{aligned}
$$

We have defined the exterior product on $\mathrm{s}^{-}$-space to satisfy the property

$$
\delta^{<\alpha>} \wedge \delta^{<\beta>}=-(-)^{|<\alpha><\beta>|} \delta^{<\beta>} \wedge \delta^{<\alpha>} .
$$




\subsection{Metrics in dvs-bundles}

The base $\tilde{M}$ of dvs-bundle $\tilde{\mathcal{E}}^{<z>}$ is considered to be a connected and paracompact s-manifold.

A metric structure on the total space $\tilde{E}^{<z>}$ of a dvs-bundle $\tilde{\mathcal{E}}^{<z>}$ is a supersymmetric, second order, covariant s-tensor field

$$
G=G_{<\alpha><\beta>} \partial^{<\alpha>} \otimes \partial^{<\beta>}
$$

which in every point $u \in \tilde{\mathcal{E}}^{<z>}$ is given by nondegenerate supersymmetric matrix $G_{<\alpha><\beta>}=G\left(\partial_{<\alpha>}, \partial_{<\beta>}\right) \quad$ (with nonvanishing superdeterminant, sdet $G \neq$ $0)$.

The metric and $\mathrm{N}$-connection structures on $\tilde{\mathcal{E}}^{<z>}$ are compatible if there are satisfied conditions:

$$
G\left(\delta_{I}, \partial_{<A>}\right)=0, G\left(\delta_{A_{f}}, \partial_{A_{p}}\right)=0, z \geq p>f \geq 1,
$$

or, in consequence,

$$
G_{I<A>}-N_{I}^{<B>} h_{<A><B>}=0, G_{A_{f} A_{p}}-N_{A_{f}}^{B_{p}} h_{A_{p} B_{p}}=0,
$$

where

$$
G_{I<A>}=G\left(\partial_{I}, \partial_{<A>}\right), G_{A_{f} A_{p}}=G\left(\partial_{A_{f}}, \partial_{A_{p}}\right) .
$$

From (34) one follows

$$
N_{I}^{<B>}=h^{<B><A>} G_{I<A>}, N_{A_{f}}^{A_{p}}=h^{A_{p} B_{p}} G_{A_{f} B_{p}}, \ldots,
$$

where matrices $h^{<A><B>}, h^{A_{p} B_{p}}, \ldots$ are respectively s-inverse to matrices

$$
h_{<A><B>}=G\left(\partial_{<A>}, \partial_{<B>}\right), h_{A_{p} B_{p}}=G\left(\partial_{A_{p}}, \partial_{B_{p}}\right) .
$$

So, in this case, the coefficients of N-connection are uniquely determined by the components of the metric on $\tilde{\mathcal{E}}^{<z>}$.

A compatible with $\mathrm{N}$-connection metric on $\tilde{\mathcal{E}}^{<z>}$ is written in irreducible form as

$$
G(X, Y)=G(h X, h Y)+G\left(v_{1} X, v_{1} Y\right)+\ldots+G\left(v_{z} X, v_{z} Y\right), \quad X, Y \in \Xi\left(\tilde{\mathcal{E}}^{<z>}\right),
$$

and looks locally as

$$
\begin{gathered}
G=g_{\alpha \beta}(u) \delta^{\alpha} \otimes \delta^{\beta}=g_{I J} d^{I} \otimes d^{J}+h_{<A><B>} \delta^{<A>} \otimes \delta^{<B>}= \\
g_{I J} d^{I} \otimes d^{J}+h_{A_{1} B_{1}} \delta^{A_{1}} \otimes \delta^{B_{1}}+h_{A_{2} B_{2}} \delta^{A_{2}} \otimes \delta^{B_{2}}+\ldots+h_{A_{z} B_{z}} \delta^{A_{z}} \otimes \delta^{B_{z}} .
\end{gathered}
$$


A d-connection $D$ on $\tilde{\mathcal{E}}^{<z>}$ is metric, or compatible with metric $G$, if conditions

$$
D_{<\alpha>} G_{<\beta><\gamma>}=0
$$

are satisfied.

A d-connection $D$ on $\tilde{\mathcal{E}}^{<z>}$ provided with a metric $G$ is a metric d-connection if and only if

$$
D_{X}^{(h)}(h G)=0, D_{X}^{(h)}\left(v_{p} G\right)=0, D_{X}^{\left(v_{p}\right)}(h G)=0, D_{X}^{\left(v_{f}\right)}\left(v_{p} G\right)=0
$$

for every $, f, p=1,2, \ldots, z$, and $X \in \Xi\left(\tilde{\mathcal{E}}^{<z>}\right)$. Conditions (36) are written in locally adapted form as

$$
g_{I J \mid K}=0, g_{I J \perp<A>}=0, h_{<A><B>\mid K}=0, h_{<A><B>\perp<C>}=0 .
$$

In every dvs-bundle provided with compatible $\mathrm{N}$-connection and metric structures one exists a metric d-connection (called the canonical d-connection associated to $G$ ) depending only on components of $\mathrm{G}$-metric and $\mathrm{N}$-connection. Its local coefficients $C \Gamma=\left(\grave{L}_{J K}^{I}, \grave{L}_{<B>K}^{<A>}, \grave{C}_{J<C>}^{I}, \grave{C}_{<B><C>}^{<A>}\right)$ are as follows:

$$
\begin{gathered}
\grave{L}_{J K}^{I}=\frac{1}{2} g^{I H}\left(\delta_{K} g_{H J}+\delta_{J} g_{H K}-\delta_{H} g_{J K}\right), \\
\grave{L}<A>K=\delta_{<B>} N_{K}^{<A>}+ \\
\frac{1}{2} h^{<A><C>}\left[\delta_{<K>} h_{<B><C>}-\left(\delta_{<B>} N_{K}^{<D>}\right) h_{<\dot{D}><C>}-\left(\delta_{<C>} N_{K}^{<D>}\right) h_{<\dot{D}><B>}\right], \\
\grave{C}_{J<C>}^{I}=\frac{1}{2} g^{I K} \delta_{<C>} g_{J K}, \\
\grave{C}_{<B><C>>}=\frac{1}{2} h^{<A><D>}\left(\delta_{<C>} h_{<D><B>}+\delta_{<B>} h_{<D><C>}-\delta_{<D>} h_{<B><C>} .\right.
\end{gathered}
$$

We emphasize that, in general, the torsion of $C \Gamma$-connection (37) does not vanish.

It should be noted here that on dvs-bundles provided with $\mathrm{N}$-connection and d-connection and metric really it is defined a multiconnection ds-structure, i.e. we can use in an equivalent geometric manner different types of d-connections with various properties. For example, for modeling of some physical processes we can use an extension of the Berwald d-connection

$$
B \Gamma=\left(L_{J K}^{I}, \delta_{<B>} N_{K}^{<A>}, 0, C_{<B><C>}^{<A>}\right),
$$


where $L_{J K}^{I}=\grave{L}_{J K}^{I}$ and $C^{<A>}{ }_{<B><C>}=\grave{C}_{<B><C>}^{<A>}$, which is hv-metric, i.e. satisfies conditions:

$$
D_{X}^{(h)} h G=0, \ldots, D_{X}^{\left(v_{p}\right)} v_{p} G=0, \ldots, D_{X}^{\left(v_{z}\right)} v_{z} G=0
$$

for every $X \in \Xi\left(\tilde{\mathcal{E}}^{<z>}\right)$, or in locally adapted coordinates,

$$
g_{I J \mid K}=0, h_{<A><B>\perp<C>}=0 .
$$

As well we can introduce the Levi-Civita connection

$$
\left\{\frac{<\alpha>}{<\beta><\gamma>}\right\}=\frac{1}{2} G^{<\alpha><\beta>}\left(\partial_{<\beta>} G_{<\tau><\gamma>}+\partial_{<\gamma>} G_{<\tau><\beta>}-\partial_{<\tau>} G_{<\beta><\gamma>}\right),
$$

constructed as in the Riemann geometry from components of metric $G_{\langle\alpha\rangle\langle\beta\rangle}$ by using partial derivations $\partial_{<\alpha>}=\frac{\partial}{\partial u^{<\alpha>}}=\left(\frac{\partial}{\partial x^{I}}, \frac{\partial}{\partial y^{<A>}}\right)$, which is metric but not a d-connection.

In our further considerations we shall largely use the Christoffel $\mathrm{d}$-symbols defined similarly as components of Levi-Civita connection but by using lapartial derivations,

$$
\tilde{\Gamma}_{<\beta><\gamma>}^{<\alpha>}=\frac{1}{2} G^{<\alpha><\tau>}\left(\delta_{<\beta>} G_{<\tau><\gamma>}+\delta_{<\gamma>} G_{<\tau><\beta>}-\delta_{<\tau>} G_{<\beta><\gamma>}\right),
$$

having components

$$
C \tilde{\Gamma}=\left(L_{J K}^{I}, 0,0, C^{<A>}{ }_{<B><C>}\right),
$$

where coefficients $L_{J K}^{I}$ and $C^{<A>}{ }_{<B><C>}$ must be computed as in formulas (38).

We can express arbitrary d-connection as a deformation of the background d-connection (38):

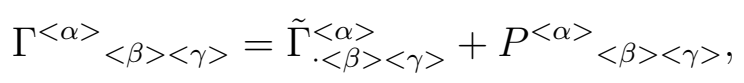

where $P^{<\alpha>}{ }_{<\beta><\gamma>}$ is called the deformation ds-tensor. Putting splitting (40) into (25) and (29) we can express torsion $T^{<\alpha>}<\beta><\gamma>$ and curvature $R_{<\beta>}{ }^{<\alpha>}{ }_{\langle\gamma\rangle<\delta>}$ of a d-connection $\Gamma^{<\alpha>}{ }_{<\beta><\gamma>}$ as respective deformations of torsion $\tilde{T}_{<\beta><\gamma>}^{<\alpha>}$ and torsion $\tilde{R}_{<\beta>\cdot<\gamma><\delta>}^{<\alpha>}$ for connection $\tilde{\Gamma}_{<\beta><\gamma>}^{<\alpha>}$ :

$$
T_{<\beta><\gamma>}^{<\alpha>}=\tilde{T}_{\cdot<\beta><\gamma>}^{<\alpha>}+\ddot{T}_{\cdot<\beta><\gamma>}^{<\alpha>}
$$

and

$$
R_{<\beta>}{ }_{<\alpha><\gamma>}=\tilde{R}_{<\beta>\cdot\langle\gamma\rangle\langle\delta>}^{<\alpha>}+\ddot{R}_{<\beta>\cdot<\gamma><\delta>}^{<\alpha>},
$$


where

$$
\begin{gathered}
\tilde{T}_{<\beta><\gamma>}^{<\alpha>}=\tilde{\Gamma}_{<\beta><\gamma>}^{<\alpha>}-(-)^{|<\beta><\gamma>|} \tilde{\Gamma}_{<\gamma><\beta>}^{<\alpha>}+w^{<\alpha>}{ }_{<\gamma><\delta>}, \\
\ddot{T}_{<\beta><\gamma>}^{<\alpha>}=\ddot{\Gamma}_{<\beta><\gamma>}^{<\alpha>}-(-)^{|<\beta><\gamma>| \ddot{\Gamma}_{<\gamma><\beta>}^{<\alpha>},}
\end{gathered}
$$

and

$$
\begin{aligned}
& \tilde{R}_{<\beta>\cdot\langle\gamma\rangle\langle\delta>}^{\cdot<\alpha>}=\delta_{<\delta>} \tilde{\Gamma}_{<\beta><\gamma>}^{<\alpha>}-(-)^{|<\gamma><\delta>|} \delta_{<\gamma>} \tilde{\Gamma}_{<\beta><\delta>}^{<\alpha>}+ \\
& \tilde{\Gamma}_{<\beta>\langle\gamma\rangle}^{<\varphi>} \tilde{\Gamma}_{<\varphi><\delta>}^{<\alpha>}-(-)^{\mid<\gamma>\langle\delta>|} \tilde{\Gamma}_{<\beta><\delta>}^{<\varphi>} \tilde{\Gamma}_{<\varphi><\gamma>}^{<\alpha>}+\tilde{\Gamma}_{<\beta><\varphi>}^{<\alpha>} w^{<\varphi><>>\delta>}, \\
& \ddot{R}_{<\beta>\cdot<\gamma><\delta>}^{<\alpha>}=\tilde{D}_{<\delta>} P^{<\alpha>}{ }_{<\beta><\gamma>}-(-)^{|<\gamma><\delta>|} \tilde{D}_{<\gamma>} P^{<\alpha><\beta><\delta>}+
\end{aligned}
$$

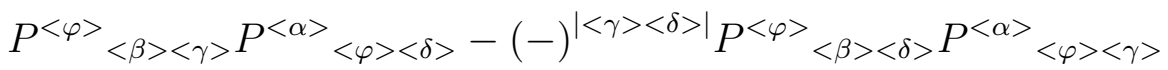

$$
\begin{aligned}
& +P^{<\alpha>}<\beta><\varphi>w^{<\varphi>}<\gamma><\delta>\text {, }
\end{aligned}
$$

the nonholonomy coefficients $w^{<\alpha>}<\beta><\gamma>$ are defined as

$$
\left[\delta_{<\alpha>}, \delta_{<\beta>}\right\}=\delta_{<\alpha>} \delta_{<\beta>}-(-)^{|<\alpha><\beta>|} \delta_{<\beta>} \delta_{<\alpha>}=w^{<\tau>}<\alpha><\beta>\delta_{<\tau>} .
$$

We emphasize that if from geometric point of view all considered $\mathrm{d}-$-connections are "equal in rights", the construction of physical models on la-spaces requires an explicit fixing of the type of $\mathrm{d}$-connection and metric structures.

\section{$5 \quad$ Higher Order Tangent S-bundles}

The aim of this section is to present a study of supersymmetric extensions from $\widetilde{M}$ to $T \tilde{M}$ and $O s c^{(z)} \widetilde{M}$ and to consider corresponding prolongations of Riemann and generalized Finsler structures (on classical and new approaches to Finsler geometry, its generalizations and applications in physics se, for example, [38, 30, 90, 75, 76, 7, 8, 74, 78, 5, 6, 22, 14]).

The presented in the previous section basic results on dvs-bundles $\tilde{\mathcal{E}}^{<z>}$ provided with $\mathrm{N}$-connection, d-connection and metric structures can be correspondingly adapted to the osculator s-bundle $\left(O s c^{z} \tilde{M}, \pi, \tilde{M}\right)$. In this case the dimension of the base space and typical higher orders fibre coincides and we shall not distinguish indices of geometrical objects.

Coefficients of a d-connection $D \Gamma(N)=\left(L_{J M}^{I}, C_{(1) J M}^{I}, \ldots, C_{(z) J M}^{I}\right)$ in $O s c^{z} \tilde{M}$, with respect to a la-base are introduced as to satisfy equations

$$
\begin{gathered}
D_{\frac{\delta}{\delta x^{I}}} \frac{\delta}{\delta y_{(f)}^{I}}=L_{I J}^{M} \frac{\delta}{\delta y_{(f)}^{M}}, D_{\frac{\delta}{\delta y_{(p)}^{J}}} \frac{\delta}{\delta y_{(f)}^{I}}=C_{(p) I J}^{M} \frac{\delta}{\delta y_{(f)}^{M}}, \\
\left(f=0,1, \ldots, z ; p=1, \ldots, z, \text { and } y_{(0)}^{I}=x^{I}\right) .
\end{gathered}
$$


A metric structure on $O s c^{z} \tilde{M}$ is ds-tensor s-symmetric field $g_{I J}\left(u_{(z)}\right)=$ $g_{I J}\left(x, y_{(1)}, y_{(2)}, \ldots, y_{(z)}\right)$ of type $(0,2), \operatorname{srank}\left|g_{i j}\right|=(n, m)$. The N-lift of Sasaki type of $g_{I J}$ is given by (see (35)) defines a global Riemannian s-structure (if $\widetilde{M}$ is a s-differentiable, paracompact s-manifold):

$$
G=g_{I J}\left(u_{(z)}\right) d x^{I} \otimes d x^{J}+g_{I J}\left(u_{(z)}\right) d y_{(1)}^{I} \otimes d y_{(1)}^{J}+\ldots+g_{I J}\left(u_{(z)}\right) d y_{(z)}^{I} \otimes d y_{(z)}^{J} .
$$

The condition of compatibility of a d-connection (41) with metric (42) is expressed as

$$
D_{X} G=0, \forall X \in \Xi\left(O s c^{z} \tilde{M}\right),
$$

or, by using $\mathrm{d}$-covariant partial derivations $\left.\right|_{(p)}$ defined by coefficients $\left(L_{J M}^{I}, C_{(1) J M}^{I}, \ldots, C_{(z) J M}^{I}\right)$,

$$
g_{I J \mid M=0,} g_{\left.I J\right|_{(p) M}}=0,(p=1, \ldots, z) .
$$

An example of compatible with metric d-connection is given by Christoffel dsymbols (see (39)):

$$
\begin{gathered}
L_{I J}^{M}=\frac{1}{2} g^{M K}\left(\frac{\delta g_{K J}}{\partial x^{I}}+\frac{\delta g_{I K}}{\partial x^{J}}-\frac{\delta g_{I J}}{\partial x^{K}}\right), \\
C_{(p) I J}^{M}=\frac{1}{2} g^{M K}\left(\frac{\delta g_{K J}}{\partial y_{(p)}^{I}}+\frac{\delta g_{I K}}{\partial y_{(p)}^{J}}-\frac{\delta g_{I J}}{\partial y_{(p)}^{K}}\right) ; p=1,2, \ldots, z .
\end{gathered}
$$

\subsection{Supersymmetric extensions of Finsler spaces}

We start our considerations with the ts-bundle $T \tilde{M}$. An s-vector $X \in \Xi(T \tilde{M})$ is decomposed with respect to la-bundles as

$$
X=X(u)^{I} \delta_{I}+Y(u)^{I} \partial_{I},
$$

where $u=u^{\alpha}=\left(x^{I}, y^{J}\right)$ local coordinates. The s-tangent structures (16) are transformed into a global map

$$
J: \Xi(T \tilde{M}) \rightarrow \Xi(T \tilde{M})
$$

which does not depend on N-connection structure:

$$
J\left(\frac{\delta}{\delta x^{I}}\right)=\frac{\partial}{\partial y^{I}}
$$

and

$$
J\left(\frac{\partial}{\partial y^{I}}\right)=0 .
$$


This endomorphism is called the natural ( or canonical ) almost tangent structure on $T \tilde{M}$; it has the properties:

$$
\text { 1) } \left.J^{2}=0, \quad 2\right) I m J=K e r J=V T \tilde{M}
$$

and 3) the Nigenhuis s-tensor,

$$
\begin{gathered}
N_{J}(X, Y)=[J X, J Y\}-J[J X, Y\}-J[X, J Y] \\
(X, Y \in \Xi(T N))
\end{gathered}
$$

identically vanishes, i.e. the natural almost tangent structure $J$ on $T \tilde{M}$ is integrable.

A generalized Lagrange superspace, GLS-space, is a pair $G L^{n, m}=\left(\tilde{M}, g_{I J}(x, y)\right)$, where $g_{I J}(x, y)$ is a ds-tensor field on $\tilde{T} \tilde{M}=T \tilde{M}-\{0\}, \quad$ s-symmetric of superrank $(n, m)$.

We call $g_{I J}$ as the fundamental ds-tensor, or metric ds-tensor, of GLS-space.

There exists an unique d-connection $C \Gamma(N)$ which is compatible with $g_{I J}(u)$ and has vanishing torsions $T_{J K}^{I}$ and $S_{J K}^{I}$ (see formulas (25) rewritten for tsbundles). This connection, depending only on $g_{I J}(u)$ and $N_{J}^{I}(u)$ is called the canonical metric d-connection of GLS-space. It has coefficients

$$
\begin{aligned}
L_{J K}^{I} & =\frac{1}{2} g^{I H}\left(\delta_{J} g_{H K}+\delta_{H} g_{J K}-\delta_{H} g_{J K}\right), \\
C_{J K}^{I} & =\frac{1}{2} g^{I H}\left(\partial_{J} g_{H K}+\partial_{H} g_{J K}-\partial_{H} g_{J K}\right) .
\end{aligned}
$$

There is a unique normal d-connection $D \Gamma(N)=\left(\bar{L}_{\cdot J K}^{I}, \bar{C}_{\cdot J K}^{I}\right)$ which is metric and has a priori given torsions $T_{J K}^{I}$ and $S_{J K}^{I}$. The coefficients of $D \Gamma(N)$ are the following ones:

$$
\begin{aligned}
& \bar{L}_{\cdot J K}^{I}=L_{J K}^{I}-\frac{1}{2} g^{I H}\left(g_{J R} T_{H K}^{R}+g_{K R} T_{H J}^{R}-g_{H R} T_{K J}^{R}\right), \\
& \bar{C}_{\cdot J K}^{I}=C_{J K}^{I}-\frac{1}{2} g^{I H}\left(g_{J R} S_{H K}^{R}+g_{K R} S_{H J}^{R}-g_{H R} S_{K J}^{R}\right),
\end{aligned}
$$

where $L^{I}{ }_{J K}$ and $C_{J K}^{I}$ are the same as for the $C \Gamma(N)$-connection (41).

The Lagrange spaces were introduced [61] in order to geometrize the concept of Lagrangian in mechanics (the Lagrange geometry is studied in details in [75, 76]). For s-spaces we present this generalization:

A Lagrange s-space, LS-space, $L^{n, m}=\left(\tilde{M}, g_{I J}\right)$, is defined as a particular case of GLS-space when the ds-metric on $\tilde{M}$ can be expressed as

$$
g_{I J}(u)=\frac{1}{2} \frac{\partial^{2} L}{\partial y^{I} \partial y^{J}},
$$


where $L: T \tilde{M} \rightarrow \Lambda$, is a s-differentiable function called a s-Lagrangian on $\tilde{M}$.

Now we consider the supersymmetric extension of Finsler space: A Finsler s-metric on $\tilde{M}$ is a function $F_{S}: T \tilde{M} \rightarrow \Lambda$ having the properties:

1. The restriction of $F_{S}$ to $T \tilde{M}=T \tilde{M} \backslash\{0\}$ is of the class $G^{\infty}$ and $\mathrm{F}$ is only supersmooth on the image of the null cross-section in the ts-bundle to $\tilde{M}$.

2. The restriction of $\mathrm{F}$ to $\tilde{T} \tilde{M}$ is positively homogeneous of degree 1 with respect to $\left(y^{I}\right)$, i.e. $F(x, \lambda y)=\lambda F(x, y)$, where $\lambda$ is a real positive number.

3. The restriction of $\mathrm{F}$ to the even subspace of $\tilde{T} \tilde{M}$ is a positive function.

4. The quadratic form on $\Lambda^{n, m}$ with the coefficients

$$
g_{I J}(u)=\frac{1}{2} \frac{\partial^{2} F^{2}}{\partial y^{I} \partial y^{J}}
$$

defined on $\tilde{T} \tilde{M}$ is nondegenerate.

A pair $F^{n, m}=(\tilde{M}, F)$ which consists from a supersmooth s-manifold $\tilde{M}$ and a Finsler s-metric is called a Finsler superspace, FS-space.

It's obvious that FS-spaces form a particular class of LS-spaces with sLagrangian $L=F^{2}$ and a particular class of GLS-spaces with metrics of type (58).

For a FS-space we can introduce the supersymmetric variant of nonlinear Cartan connection [30, 90]:

$$
N_{J}^{I}(x, y)=\frac{\partial}{\partial y^{J}} G^{* I}
$$

where

$$
G^{* I}=\frac{1}{4} g^{* I J}\left(\frac{\partial^{2} \varepsilon}{\partial y^{I} \partial x^{K}} y^{K}-\frac{\partial \varepsilon}{\partial x^{J}}\right), \quad \varepsilon(u)=g_{I J}(u) y^{I} y^{J},
$$

and $g^{* I J}$ is inverse to $g_{I J}^{*}(u)=\frac{1}{2} \frac{\partial^{2} \varepsilon}{\partial y^{I} \partial y^{J}}$. In this case the coefficients of canonical metric d-connection (25) gives the supersymmetric variants of coefficients of the Cartan connection of Finsler spaces. A similar remark applies to the Lagrange superspaces.

\subsection{Higher order prolongations of Riemann,Finsler and Lagrange $\mathrm{s}-$-spaces}

The geometric constructions on $T \widetilde{M}$ from the previus subsection have corresponding generalizations to the $O s c^{(z)} \widetilde{M}$ s-bundle. The basic idea is similar to that used for prolongations of geometric structures (see [79] for prolongations on tangent bundle). Having defined a metric structure $g_{I J}(x)$ on a s-manifold $\widetilde{M}$ we can extend it to the $O s c^{z} \tilde{M}$ s-bundle by considering $g_{I J}\left(u_{(z)}\right)=g_{I J}(x)$ 
in (42). R. Miron and Gh. Atanasiu [77] solved the problem of prolongations of Finsler and Lagrange structures on osculator bundle. In this subsection we shall analyze supersymmetric extensions of Finsler and Lagrange structures as well present a brief introduction into geometry of higher order Lagrange s-spaces.

Let $F^{n, m}=(\tilde{M}, F)$ be a FS-space with the fundamental function $F_{S}$ : $T \tilde{M} \rightarrow \Lambda$ on $\widetilde{M}$. A prolongation of $F$ on $O s c^{z} \tilde{M}$ is given by a map

$$
\left(F \circ \pi_{1}^{z}\right)\left(u_{(z)}\right)=F\left(u_{(1)}\right)
$$

and corresponding fundamental tensor

$$
g_{I J}\left(u_{(1)}\right)=\frac{1}{2} \frac{\partial^{2} F^{2}}{\partial y_{(1)}^{I} \partial y_{(1)}^{J}},
$$

for which

$$
\left(g_{I J} \circ \pi_{1}^{z}\right)\left(u_{(z)}\right)=g_{I J}\left(u_{(1)}\right) .
$$

So, $g_{I J}\left(u_{(1)}\right)$ is a ds-tensor on

$O \operatorname{sk}^{z} \widetilde{M}=O s c^{z} \tilde{M} /\{0\}=\left\{\left(u_{(z)}\right) \in O s c^{z} \tilde{M}, \operatorname{srank}\left|y_{(1)}^{I}\right|=1\right\}$.

The Christoffel d-symbols

$$
\gamma_{I J}^{M}\left(u^{(1)}\right)=\frac{1}{2} g^{M K}\left(u_{(1)}\right)\left(\frac{\partial g_{K I}\left(u_{(1)}\right)}{\partial x^{J}}+\frac{\partial g_{J K}\left(u_{(1)}\right)}{\partial x^{I}}-\frac{\partial g_{I J}\left(u_{(1)}\right)}{\partial x^{K}}\right)
$$

define the Cartan nonlinear connection [29]:

$$
G_{(N) J}^{I}=\frac{1}{2} \frac{\partial}{\partial y_{(1)}^{J}}\left(\gamma_{K M}^{I} y_{(1)}^{K} y_{(1)}^{M}\right) .
$$

The dual coefficients for the N-connection (21) are recurrently computed by using (44) and operator

$$
\begin{gathered}
\Gamma=y_{(1)}^{I} \frac{\partial}{\partial x^{I}}+2 y_{(2)}^{I} \frac{\partial}{\partial y_{(1)}^{I}}+\ldots+z y_{(z)}^{I} \frac{\partial}{\partial y_{(z-1)}^{I}} \\
M_{(1) J}^{I}=G_{(N) J}^{I}, \\
M_{(2) J}^{I}=\frac{1}{2}\left[\Gamma G_{(N) J}^{I}+G_{(N) K}^{I} M_{(1) J}^{K}\right] \\
\ldots \ldots \ldots \ldots . \\
M_{(z) J}^{I}=\frac{1}{z}\left[\Gamma M_{(z-1) J}^{I}+G_{(N) K}^{I} M_{(z-1) J}^{K}\right] .
\end{gathered}
$$

The prolongations of FS-spaces can be generalized for Lagrange s-spaces (on Lagrange spaces and theirs higher order extensions see [75, 76, 77] and on supersymmetric extensions of Finsler geometry see $\| 106])$. Let $L^{n, m}=\left(\tilde{M}, g_{I J}\right)$ be a Lagrange s-space. The Lagrangian $L: T \widetilde{M} \rightarrow \Lambda$ can be extended on $O s c^{z} \tilde{M}$ by using maps of the Lagrangian, $\left(L \circ \pi_{1}^{z}\right)\left(u_{(z)}\right)=L\left(u_{(1)}\right)$, and, as a consequence, of the fundamental tensor $(43),\left(g_{I J} \circ \pi_{1}^{z}\right)\left(u_{(z)}\right)=g_{I J}\left(u_{(1)}\right)$. 


\subsection{Higher order Lagrange $\mathrm{s}-$-spaces}

We introduce the notion of Lagrangian of $\mathrm{z}$-order on a differentiable $\mathrm{s}$-manifold $\widetilde{M}$ as a map $L^{z}: O s c^{z} \tilde{M} \rightarrow \Lambda$. In order to have concordance with the definitions proposed by [77] we require the even part of the fundamental ds-tensor to be of constant signature. Here we also note that questions to considered in this subsection, being an supersymmetric approach, are connected with the problem of elaboration of the so-called higher order analytic mechanics (see, for instance, [34, 70, 69, 94]).

A Lagrangian s-differentiable of order $z(z=1,2,3, \ldots)$ on s-differentiable s-manifold $\widetilde{M}$ is an application $L^{(z)}: O s c^{z} \widetilde{M} \rightarrow \Lambda$, s-differentiable on $O s k^{z} \widetilde{M}$ and smooth in the points of $O s c^{z} \widetilde{M}$ where $y_{(1)}^{I}=0$.

It is obvious that

$$
g_{I J}\left(x, y_{(1)}, \ldots, y_{(z)}\right)=\frac{1}{2} \frac{\partial^{2} L^{(z)}}{\partial y_{(z)}^{I} \partial y_{(z)}^{J}}
$$

is a ds-tensor field because with respect to coordinate transforms (3) one holds transforms

$$
K_{I}^{I^{\prime}} K_{J}^{J^{\prime}} g_{I^{\prime} J^{\prime}}=g_{I J}
$$

A Lagrangian $L$ is regular if $\operatorname{srank}\left|g_{I J}\right|=(n, m)$.

A Lagrange s-space of $z$-order is a pair $L^{(z, n, m)}=\left(\widetilde{M}, L^{(z)}\right)$, where $L^{(z)}$ is a $\mathrm{s}$-differentiable regular Lagrangian of $z$-order, and with ds-tensor $g_{I J}$ being of constant signature on the even part of the basic s-manifold.

For details on nonsupersymmetric osculator bundles we cite [77]

\section{Superstrings in Higher Order Anisotropic S- Spaces}

This section considers the basic formalism for superstrings in dvs-bundles. We shall begin our study with nonsupersymmetric two dimensional higher order anisotropic sigma models. Then we shall analyze supersimmetric extensions and locally anisotropic generalizations of the Green-Schvarz action.

\subsection{Two dimensional higher order anisotropic sigma $\mathrm{s}^{-}$ models}

Let $\widehat{\mathcal{E}}^{<z>}$ be a higher order anisotropic space (not superspace) with coordinates $\widehat{u}^{<\alpha>}=\widehat{u}(z)=\left(\widehat{x}^{i}=\widehat{x}(z), \widehat{y}^{<a>}=\widehat{y}(z)\right)=\left(x^{i}, y^{a_{1}}, \ldots, y^{a_{p}}, \ldots, y^{a_{z}}\right), \mathrm{d}-$ metric $\widehat{g}_{<\alpha><\beta>}$; we use denotation $\left(N_{2}, \gamma_{\ddot{a} e ̈}\right)$ for a two dimensional world sheet with 
metric $\gamma_{\ddot{a} \ddot{e}}\left(z^{\ddot{u}}\right)$ of signature $(+,-)$ and local coordinates $z=z^{\ddot{u}}$, where $\ddot{a}, \ddot{e}, \ddot{u}, \ldots=$ 1,2 .

The action of a bosonic string in a $d v$-bundle $\widehat{\mathcal{E}}^{<z>}$ is postulated as

$$
I_{\sigma}=\frac{1}{\lambda^{2}} \int d^{2} z\left\{\frac{1}{2} \sqrt{\gamma} \gamma^{\ddot{e} \ddot{e}} \partial_{\ddot{a}} \widehat{u}^{<\alpha>}(z) \partial_{\ddot{e}} \widehat{u}^{<\beta>}(z)\right\}
$$

which defines the so called two dimensional sigma model ( $\sigma$-model $)$ with $\mathrm{d}-$ metric $\widehat{g}_{\langle\alpha\rangle<\beta>}$ in higher order anisotropic spaces (dv-bundles with $\mathrm{N}$-connection) and $\lambda$ being constant. A detailed study of different modifications of the model (45) is given in also [107, 108]. Here we shall consider a supersymmetric generalization of the string action by applying the techniques of two dimensional $(1,1)$-supersymmetry by changing of scalar fields $u(z)$ into real $\mathrm{N}=1 \mathrm{~s}$-fields (without constraints; for locally isotropic constructions see [11, 23, 36, 42, 51, 83] $\widehat{u}(z, \theta)$ which are polynoms with respect to Maiorana anticommuting spinor coordinate $\theta$ :

$$
\widehat{u}^{<\alpha>}(z, \theta)=\widehat{u}^{<\alpha>}(z)+\bar{\theta} \lambda^{<\alpha>}(z)+\frac{1}{2} \bar{\theta} \theta F^{<\alpha>}(z) .
$$

We adopt next conventions and denotations with respect to two-dimension Dirac matrices $\gamma_{\ddot{a}}$ and matrix of charge conjugation $C$ :

$$
\begin{aligned}
& \left\{\gamma_{\ddot{a}}, \gamma_{\ddot{e}}\right\}=2 \eta_{\ddot{a} \ddot{e}} \mathbf{1}, \operatorname{tr}\left(\gamma_{\ddot{e}} \gamma_{\ddot{a}}\right)=2 \eta_{\ddot{e} \ddot{a}}, \quad\left(\gamma_{5}\right)^{2}=1 \\
& \widehat{\partial}=\gamma^{\ddot{e}} \partial_{\ddot{e}} ; C \gamma_{\ddot{a}}^{T} C^{-1}=-\gamma_{\ddot{a}}, C=-C^{T}=C^{-1} .
\end{aligned}
$$

For Maiorana spinors $\theta_{\tilde{a}}, \chi_{\tilde{n}}, \ldots$ one holds relations

$$
\begin{gathered}
\bar{\theta}=\theta^{+} \gamma^{0}, \overline{\theta^{\tilde{a}}}=C^{\tilde{a} \tilde{n}} \theta_{\tilde{n}}, \\
\bar{\theta} \chi=\bar{\chi} \theta, \bar{\theta} \gamma_{\ddot{a}} \chi=-\bar{\chi} \gamma_{\ddot{a}} \theta, \bar{\theta} \gamma_{5} \chi=-\bar{\chi} \gamma_{5} \theta .
\end{gathered}
$$

Let introduce in the two dimensional $(1,1)$-superspace the covariant derivations

$$
D_{\tilde{n}}=\frac{\partial}{\partial \bar{\theta}^{\tilde{n}}}-i(\widehat{\partial} \theta)_{\tilde{n}}
$$

satisfying algebra

$$
\left\{D_{\tilde{n}}, D_{\tilde{o}}\right\}=2 i(\widehat{\partial} C)_{\tilde{n} \tilde{o}} \equiv 2 i \partial_{\tilde{n} \tilde{o}}
$$

and the integration measure on anticommuting variables with properties

$$
\int d \theta_{\tilde{n}}=0, \int d \theta_{\tilde{n}} \theta^{\tilde{a}}=\delta_{\tilde{n}}^{\tilde{a}}, \frac{1}{2 i} \int d^{2} \theta(\bar{\theta} \theta)=1 .
$$


The (1,1)-supersymmetric generalization of (45) in terms of s-fields (46) is written as

$$
I_{\sigma S}=\frac{1}{8 i \pi \alpha^{\prime}} \int d^{2} z \int d^{2} \theta\left\{\widehat{g}_{<\alpha><\beta>}(\widehat{u})-\widehat{b}_{<\alpha><\beta>}(\widehat{u})\right\} \bar{D} \widehat{u}^{<\alpha>}\left(1+\gamma_{5}\right) D \widehat{u}^{<\beta>},
$$

where $\lambda^{2}=2 \pi \alpha^{\prime}$ which is a higher order anisotropic generalization of the Curtright-Zachos [36 nonlinear sigma model. Integrating on $\theta$ and excluding auxiliary fields $F^{<\alpha>}$ according to theirs algebraic equations we obtain from the last expression:

$$
\begin{aligned}
& I_{\sigma S}=\frac{1}{2} \int d^{2} z\left\{\widehat{g}_{<\alpha><\beta>} \partial^{\ddot{e}} \widehat{u}^{<\alpha>} \partial_{\ddot{e}} \widehat{u}^{<\beta>}+\right. \\
& \varepsilon^{\dddot{e} i} \widehat{b}_{<\alpha><\beta>} \partial_{\ddot{e}} \widehat{u}^{<\alpha>} \partial_{i} \widehat{u}^{<\beta>}+i \widehat{g}_{<\alpha><\beta>} \bar{\lambda}^{<\alpha>} \gamma^{\ddot{e}} \widehat{D}_{\ddot{e}}^{(-)} \lambda^{<\beta>}+ \\
& \left.\frac{1}{8} \widehat{R}_{<\beta><\alpha><\gamma><\delta>}^{(-)} \bar{\lambda}^{<\alpha>}\left(1+\gamma_{5}\right) \lambda^{<\gamma>} \bar{\lambda}^{<\beta>}\left(1+\gamma_{5}\right) \lambda^{<\delta>}\right\},
\end{aligned}
$$

where

$$
\widehat{D}_{\ddot{e}}^{( \pm)} \lambda^{<\beta>}=\left[\delta_{<\alpha>}^{<\beta>} \partial_{\ddot{e}}+\widehat{\widetilde{\Gamma}}_{<\alpha><\gamma>}^{<\beta>} \partial_{\ddot{e}} \widehat{u}^{<\gamma>} \pm \widehat{B}_{<\alpha><\gamma>}^{<\beta>} \varepsilon_{\ddot{e} \ddot{o}} \partial^{\ddot{o}} \widehat{u}^{<\gamma>}\right] \lambda^{<\gamma>}
$$

$\widehat{\widetilde{\Gamma}}_{<\alpha><\gamma>}^{<\beta>}$ are Christoffel d-symbols (39) on dv-bundle. In order to have compatible with the $\mathrm{N}$-connection structure motions of la-strings we consider [107, 108] these relations between ds-tensor $b_{<\alpha><\beta>}$, strength $\widehat{B}_{<\alpha><\beta><\gamma>}=\delta_{[<\alpha>} \widehat{b}_{<\beta><\gamma>\}}$ and torsion $T_{<\alpha><\gamma>}^{<\beta>}($ see $(29))$ :

$$
\delta_{<\alpha>} \widehat{b}_{<\beta><\gamma>}=\widehat{g}_{<\alpha><\delta>} \widehat{T}_{<\beta><\gamma>}^{<\delta>},
$$

with s-integrability conditions

$$
\Omega_{a_{p} a_{s}}^{a_{f}} \delta_{a_{f}} \widehat{b}_{<\beta><\gamma>}=\delta_{\left[a_{h}\right.} \widehat{T}_{\left.a_{s}\right\}<\beta><\gamma>},(f<p, s ; p, s=0,1, \ldots, z)
$$

where $\Omega_{a_{p} a_{s}}^{a_{f}}$ are the coefficients of the $\mathrm{N}$-connection curvature (11). In this case we can express $\widehat{B}_{\langle\alpha\rangle<\beta><\gamma>}=\widehat{T}_{[<\alpha><\beta><\gamma>\}}$. Conditions (48) and (49) define a model of higher order anisotropic superstrings when the $\sigma$-modes santisymmetric strength is introduced from the higher order anisotropic background torsion. More general constructions are possible by using normal coordinates locally adapted to both $\mathrm{N}$-connection and torsion structures on background s-spaces. For simplicity, we omit such considerations in this work.

Ds-tensor $\widehat{R}_{<\alpha><\beta><\gamma><\delta>}$ from (47) denotes the curvature with torsion $B$ :

$$
\widehat{R}_{<\beta><\alpha><\gamma><\delta>}^{( \pm)}\left[\widehat{\Gamma}^{( \pm)}\right]=\widetilde{R}_{<\beta><\alpha><\gamma><\delta>} \mp
$$




$$
\begin{aligned}
& D_{\langle\gamma\rangle} \widehat{B}_{\langle\alpha\rangle\langle\beta\rangle\langle\delta\rangle} \pm D_{<\delta>} \widehat{B}_{\langle\alpha\rangle\langle\beta\rangle\langle\gamma\rangle}+ \\
& \widehat{B}_{\langle\tau\rangle\langle\alpha\rangle\langle\gamma\rangle} \widehat{B}_{\langle\delta\rangle\langle\beta\rangle}^{<\tau>}-\widehat{B}_{\langle\tau\rangle\langle\alpha\rangle\langle\delta\rangle} \widehat{B}_{\langle\gamma\rangle\langle\beta\rangle}^{<\tau>},
\end{aligned}
$$

where $\widetilde{R}_{\langle\beta\rangle\langle\alpha\rangle\langle\gamma\rangle\langle\delta>}$ is the curvature of the torsionless Christoffel d-symbols (39),

$$
\begin{gathered}
\widehat{\Gamma}_{<\beta><\gamma>}^{<\alpha>( \pm)}=\widehat{g}^{<\alpha><\tau>} \widehat{\Gamma}_{<\tau><\beta><\gamma>}^{( \pm)}, \widehat{\Gamma}_{<\tau><\beta><\gamma>}^{( \pm)}=\widehat{\widetilde{\Gamma}}_{<\tau><\beta><\gamma>} \pm B_{<\tau><\beta><\gamma>}^{( \pm)}, \\
\widetilde{\Gamma}_{<\tau><\beta><\gamma>}=\frac{1}{2}\left(\delta_{<\beta>} \widetilde{g}_{<\tau><\gamma>}+\delta_{<\gamma>} \widetilde{g}_{<\beta><\tau>}-\delta_{<\tau>} \widetilde{g}_{<\beta><\gamma>}\right) .
\end{gathered}
$$

In order to define a locally supersymmetric generalization of the model (47) we consider a supersymmetric calculus of the set of $(1,1)$-multiplets of higher order anisotropic matter $\left(\hat{\varphi}^{\langle\alpha\rangle}, \hat{\lambda}^{\langle\alpha\rangle}=\lambda^{\langle\alpha\rangle}\right)$ with the multiplet of $(1,1)$ supergravity $\left(e_{\ddot{e}}^{\ddot{e}}, \psi_{\ddot{e}}\right)$ in two dimensions (see [62, 63, 64 for locally isotropic constructions).

The global supersymmetric variant of action (47), for $2 \pi \alpha^{\prime}=1$, is written as

$$
\begin{aligned}
& I_{0}[\varphi, \lambda]=\frac{1}{2} \int d^{2} z\left[\widehat{g}_{<\alpha><\beta>} \partial^{\ddot{\ddot{e}} \widehat{u}^{<\alpha>}} \partial_{\underline{\ddot{e}} \widehat{u}^{<\beta>}}+\widehat{b}_{<\alpha><\beta>} \varepsilon^{\underline{\ddot{e}} \underline{\ddot{a}}} \partial_{\underline{\underline{e}}} \widehat{u}^{<\alpha>} \partial_{\underline{\underline{a}}} \widehat{u}^{<\beta>}+\right. \\
& i \widehat{g}_{\langle\alpha><\beta>} \bar{\lambda}^{<\alpha>} \gamma^{\underline{\underline{e}}}\left(D_{\underline{e}} \lambda\right)^{<\beta>}+i \widehat{B}_{<\alpha><\beta><\gamma>} \bar{\lambda}^{<\alpha>} \gamma_{5} \gamma^{\underline{\underline{e}}}\left(\partial_{\underline{\ddot{e}}} \widehat{u}^{<\beta>}\right) \lambda^{<\gamma>}+ \\
& \frac{1}{6} \widehat{\widetilde{R}}_{\langle\beta>\langle\alpha><\gamma><\delta>}\left(\bar{\lambda}^{<\alpha>} \lambda^{<\gamma>}\right)\left(\bar{\lambda}^{<\beta>} \lambda^{<\delta>}\right)- \\
& \frac{1}{4} \widehat{\widetilde{D}}_{\langle\varepsilon\rangle} B_{\langle\alpha\rangle<\beta><\tau>}\left(\bar{\lambda}^{<\alpha>} \gamma_{5} \lambda^{<\beta>}\right)\left(\bar{\lambda}^{<\tau>} \lambda^{<\varepsilon>}\right)- \\
& \left.\frac{1}{4} \widetilde{B}_{\langle\alpha\rangle\langle\beta>\langle\tau\rangle} \widetilde{B}_{<\gamma><\delta>}^{<\tau>}\left(\bar{\lambda}^{<\alpha>} \gamma_{5} \lambda^{<\beta>}\right)\left(\bar{\lambda}^{<\gamma>} \gamma_{5} \lambda^{<\delta>}\right)\right],
\end{aligned}
$$

where covariant derivation $\widehat{\widetilde{D}}_{<\varepsilon>}$ is defined by torsionless Christoffel d-symbols.

The action (50) is invariant under global s-transforms with Maiorana spinor parameter $\varepsilon$ :

$$
\begin{gathered}
\triangle \hat{\varphi}^{<\alpha>}=\bar{\varepsilon} \lambda^{<\alpha>}, \\
\triangle \lambda^{\langle\alpha>}=-i\left(\hat{\partial}^{<\alpha>}\right) \varepsilon+\frac{\varepsilon}{2}\left(\widehat{\widetilde{\Gamma}}_{<\beta>\langle\gamma>}^{<\alpha>} \bar{\lambda}^{<\beta>} \lambda^{<\gamma>}-\widetilde{B}_{<\beta>\langle\gamma>}^{<\alpha>} \bar{\lambda}^{<\beta>} \gamma_{5} \lambda^{<\gamma>}\right) .
\end{gathered}
$$

Defining Maiorana-Weyl spinors $\lambda_{ \pm}^{<\alpha>}$ (MW-spinors) instead of $\lambda^{<\alpha>}$ we can rewrite the action (50) in a more convenient form:

$$
\begin{aligned}
& I_{0}\left[\varphi, \lambda_{ \pm}\right]=\frac{1}{2} \int d^{2} z\left[\widetilde{g}_{\langle\alpha><\beta>} \partial^{\ddot{\ddot{e}}} \widetilde{u}^{<\alpha>} \partial_{\underline{\underline{e}}} \widetilde{u}^{<\beta>}+\widehat{b}_{\langle\alpha><\beta>} \varepsilon^{\underline{\underline{e}} \underline{\ddot{e}}} \partial_{\underline{\underline{e}}} \widehat{u}^{<\alpha>} \partial_{\underline{\underline{a}}} \widehat{u}^{<\beta>}+\right. \\
& i \widehat{g}_{\langle\alpha\rangle\langle\beta\rangle} \bar{\lambda}_{+}^{<\alpha>}\left(\widehat{D}^{+} \lambda_{+}\right)^{<\beta>}+i \widehat{g}_{\langle\alpha\rangle<\beta>} \bar{\lambda}_{-}^{<\alpha>}\left(\widehat{D}^{-} \lambda_{-}\right)^{<\beta>}+
\end{aligned}
$$




$$
\left.\frac{1}{4} \widehat{\widetilde{R}}_{<\beta><\alpha><\gamma><\delta>}^{+}\left(\bar{\lambda}^{<\alpha>} \gamma_{5} \lambda^{<\beta>}\right)\left(\bar{\lambda}^{<\gamma>} \gamma_{5} \lambda^{<\delta>}\right)\right]
$$

with s-symmetric transformation law

$$
\begin{gathered}
\triangle \varphi^{<\alpha>}=\triangle_{+} \varphi^{<\alpha>}+\triangle_{-} \varphi^{<\alpha>}=\bar{\varepsilon}_{+} \lambda_{-}^{<\alpha>}+\bar{\varepsilon}_{-} \lambda_{+}^{<\alpha>}, \\
\triangle \lambda_{ \pm}^{<\alpha>}=-i\left(\widehat{\partial} u^{<\alpha>}\right) \varepsilon_{\mp}-\widetilde{\Gamma}_{<\beta><\gamma>}^{<\alpha>( \pm)} \lambda_{ \pm}^{<\beta>} \triangle_{ \pm} \varphi^{<\gamma>} .
\end{gathered}
$$

For simplicity, we shall omit "hats" on geometrical objects if ambiguities connected with indices for manifolds and supermanifolds will not arise.

In string theories one considers variations of actions of type (50) with respect to s-symmetric transformation laws and decompositions with respect to powers of $\lambda$. Coefficients proportional to $\lambda^{5}$ vanishes because they do not contain derivations of $\varepsilon(z)$-parameters. In order to compensate the therms proportional to $\lambda$ and $\lambda^{3}$ one adds the so-called Nether term

$$
\begin{aligned}
& I^{(N)}=\frac{1}{2} \int d^{2} z\left[2 g_{<\alpha><\beta>}\left(\partial_{\underline{\ddot{e}}} \varphi^{<\alpha>}\right)\left(\bar{\lambda}^{<\beta>} \gamma^{\underline{\ddot{ }}} \gamma^{\underline{\ddot{e}}} \psi_{\underline{\ddot{o}}}\right)-\right. \\
& \frac{i}{3} B_{<\alpha><\beta><\gamma>}\left(\bar{\lambda}^{<\alpha>} \gamma_{5} \gamma^{\underline{\underline{e}}} \lambda^{<\beta>}\right)\left(\bar{\lambda}^{<\gamma>} \psi_{\underline{\ddot{e}}}\right)- \\
& \frac{i}{3} B_{<\alpha><\beta><\gamma>}\left(\bar{\lambda}^{<\alpha>} \gamma^{\underline{\underline{e}}} \lambda^{<\beta>}\right)\left(\bar{\lambda}^{<\gamma>} \gamma_{5} \psi_{\underline{\underline{e}}}\right),
\end{aligned}
$$

where $\psi_{\underline{e}}$ is the higher order anisotropic generalization of Maiorana gravitino with s-symmetric transformation law

$$
\triangle \psi_{\ddot{\ddot{e}}}=-\partial_{\underline{\ddot{e}}} \varepsilon+\ldots
$$

From the standard variation, but locally adapted to the $\mathrm{N}$-connection, of the $I^{(N)}$ with a next covariantization (with respect to $\left(e_{\ddot{e}}^{\ddot{e}}, \psi_{\ddot{e}}\right)$ ) of the theory. In result (it's convenient to use MW-spinors) we introduce this action:

$$
\begin{aligned}
& I=\frac{1}{2} \int d^{2} z e\left[\gamma^{\ddot{a} \ddot{u}} g_{<\alpha><\beta>} \partial_{\ddot{a}} u^{<\alpha>} \partial_{\ddot{u}} u^{<\beta>}+e^{-1} \varepsilon^{\ddot{a} \ddot{u}} b_{<\alpha><\beta>} \partial_{\ddot{a}} u^{<\alpha>} \partial_{\ddot{u}} u^{<\beta>}+\right. \\
& i g_{<\alpha><\beta>} \bar{\lambda}_{+}^{<\alpha>}\left(\widehat{D}^{+} \lambda_{+}\right)^{<\beta>}+i g_{<\alpha><\beta>} \bar{\lambda}_{-}^{<\alpha>}\left(\widehat{D}^{-} \lambda_{-}\right)^{<\beta>}+ \\
& \left.\frac{1}{4} \widetilde{R}_{<\beta><\alpha><\gamma><\delta>}^{+}\left(\bar{\lambda}^{<\alpha>} \gamma_{5} \lambda^{<\beta>}\right)\left(\bar{\lambda}^{<\gamma>} \gamma_{5} \lambda^{<\delta>}\right)\right]+ \\
& g_{<\alpha><\beta>}\left(2 \partial_{\ddot{a}} u^{<\alpha>}+\bar{\psi}_{\ddot{a}} \lambda_{+}^{<\alpha>}+\bar{\psi}_{\ddot{a}} \lambda_{-}^{<\alpha>}\right) \times \\
& \left(\bar{\lambda}_{+}^{<\beta>}\left\{\gamma^{\ddot{a} \ddot{u}}+e^{-1} \varepsilon^{\ddot{a} \ddot{u}}\right\} \psi_{\ddot{u}}+\left(\bar{\lambda}_{-}^{<\beta>}\left\{\gamma^{\ddot{a} \ddot{u}}-e^{-1} \varepsilon^{\ddot{a} \ddot{u}}\right\} \psi_{\ddot{u}}\right)+\right. \\
& \left.\frac{2 i}{3} B_{<\alpha><\beta><\gamma>}\left\{\left(\bar{\lambda}_{+}^{<\alpha>} \gamma^{\ddot{a}} \bar{\lambda}_{+}^{<\beta>}\right)\left(\bar{\lambda}_{+}^{<\gamma>} \psi_{\ddot{a}}\right)-\left(\bar{\lambda}_{-}^{<\alpha>} \gamma^{\ddot{a}} \bar{\lambda}_{-}^{<\beta>}\right)\left(\bar{\lambda}_{-}^{<\gamma>} \psi_{\ddot{a}}\right)\right\}\right],
\end{aligned}
$$


where $e=\operatorname{det}\left|e_{\ddot{e}}^{\ddot{e}}\right|$, for which the higher order anisotropic laws of supersymmetric transforms holds:

$$
\begin{gathered}
\triangle e_{\ddot{e}}^{\ddot{e}}=2 i \bar{\varepsilon} \gamma^{\underline{\underline{e}}} \psi_{\ddot{e}}, \triangle \psi_{\ddot{e}}=-D_{\ddot{e} \varepsilon}, \\
\triangle \varphi^{<\alpha>}=\triangle_{+} \varphi^{<\alpha>}+\triangle_{-} \varphi^{<\alpha>}=\bar{\varepsilon}_{+} \lambda_{-}^{<\alpha>}+\bar{\varepsilon}_{-} \lambda_{+}^{<\alpha>}, \\
\triangle \lambda_{ \pm}^{<\alpha>}=-i\left(\widehat{\partial} u^{<\alpha>}+\left\{\bar{\lambda}_{+}^{<\alpha>} \psi_{\ddot{a}}+\bar{\lambda}_{-}^{<\alpha>} \psi_{\ddot{a}}\right\} \gamma^{\ddot{a}}\right) \varepsilon_{\mp}-\widetilde{\Gamma}_{<\beta><\gamma>}^{<\alpha>( \pm)} \lambda_{ \pm}^{<\beta>} \triangle_{ \pm} \varphi^{<\gamma>} .
\end{gathered}
$$

Restricting our considerations in (51) and (52) only with $\lambda_{+}^{<\alpha>}$-spinors and $\varepsilon_{-}$-parameters, when $\varepsilon_{+}, \lambda_{-}^{<\alpha>}=0$, we obtain the action for the heterotic higher order anisotropic string on background $\left(g_{\langle\alpha\rangle<\beta>}, b_{\langle\alpha\rangle<\beta>}\right)$ with $(1,0)$-local supersymmetry $\left(\psi_{\ddot{a}} \rightarrow \psi_{\ddot{a}(-)}\right)$. This action can be interpreted as the "minimal" interaction of the higher order anisotropic $(1,0)-\operatorname{matter}\left(\varphi^{<\alpha>}, \lambda_{+}^{<\alpha>}\right)$ with $(1,0)-$ supergravity $\left(e \stackrel{\ddot{e}}{\ddot{e}}, \psi_{\ddot{e}}\right)$.

\subsection{Locally anisotropic heterotic strings}

As an illustration of application of s-field methods in locally anisotropic sspaces we shall construct the action for a model of higher order anisotropic s-string.

The (1,0)-superspaces can be parametrized by two Bose coordinates $\left(z^{\ddagger}, z^{=}\right)$ and one Fermy coordinate $\theta^{+} ; \ddot{u}=\left(z^{\ddagger}, z^{=}, \theta^{+}\right)$. One represents vector indices as $(++,--) \equiv(\ddagger,=)$ taking into account that by $(+,-)$ there are denoted spirality $\pm 1 / 2$.

The standard derivations

$$
D_{\ddot{A}}=\left\{D_{+}, \partial_{\ddagger}, \partial_{=}\right\} ; D_{+}=\frac{\partial}{\partial \theta^{+}}+i \theta^{+} \partial_{+},
$$

in the flat $(1,0)$-superspace 91] satisfy algebra

$$
\left\{D_{+}, D_{+}\right\}=2 i \partial_{\ddagger}, \partial_{\ddagger} \partial_{=}=\square,\left[\partial_{\underline{a}}, D_{+}\right]=\left[\partial_{\underline{a}}, \partial_{\underline{b}}\right]=0,
$$

and s-space integration measure

$$
\int d \theta_{+}=\frac{\partial}{\partial \theta^{+}}, d^{3} \ddot{u}^{-}=d^{2} z d \theta_{+} .
$$

In the flat $(1,0)$-superspace one defines scalar and spinor s-fields

$$
\varphi(z, \theta)=A(z)+\theta^{+} \lambda_{+}(z), \psi_{-}(z, \theta)=\eta_{-}(z)+\theta^{+} F(z)
$$

and action

$$
I=\int d^{3} \ddot{u} L=\int d^{2} z\left(D_{+} L\right)_{\mid \theta=0}
$$


with a charged Lagrangian, $L=L_{-}$, in order to have the Lorentz invariance.

The (1,0)-multiplet of supergravity is described by a set of covariant derivations

$$
\nabla_{\ddot{A}}=E_{\ddot{A}}^{\ddot{U}} D_{\ddot{U}}+\omega_{\ddot{A}}^{(M)} \equiv E_{\ddot{A}}+\Omega_{\ddot{A}},
$$

where $E_{\ddot{A}}^{\ddot{U}}$ is a s-vielbein and $\omega_{\ddot{A}}^{(M)}$ is the Lorentz connection with L-generator $M$ :

$$
\left[M, \lambda_{ \pm}\right]= \pm \frac{1}{2} \lambda_{ \pm}
$$

The covariant constraints in s-space (for (1,0)-supergravity [24, 40]) are given by relations:

$$
\begin{gathered}
\left\{\nabla_{+}, \nabla_{+}\right\}=2 i \nabla_{\ddagger},\left[\nabla_{+}, \nabla_{=}\right]=-2 i \Sigma^{+} M, \\
{\left[\nabla_{+}, \nabla_{\ddagger}\right]=0,\left[\nabla_{\ddagger}, \nabla_{=}\right]=-\Sigma^{+} \nabla_{+}-R^{(2)} M,}
\end{gathered}
$$

where $R^{(2)}=2 \nabla_{+} \Sigma^{+}$and $\Sigma^{+}$defines the covariant strength of $(1,0)$-supergravity in $\mathrm{s}-$-space.

As a consequence of $(54)$ only $E_{+}^{=}, E_{=}^{\ddagger}, E_{+}^{\ddagger}, E_{+}^{+}$and $E_{=}^{=}$are independent s-fields; the rest of components of vielbein and connection can be expressed through them. The conditions of covariance of derivations (54) lead to these transformation laws with respect to d-coordinate and local Lorentz transforms with corresponding parameters $K \underline{\ddot{U}}$ and $\Lambda_{l}$ :

$$
\nabla_{\ddot{A}}^{\prime}=e^{K} \nabla_{\ddot{A}} e^{-K}, K=K^{\ddot{U}} D_{\ddot{\underline{U}}}+\Lambda_{l} M ;
$$

for vielbeins we have

$$
\triangle E_{\ddot{A}}^{\ddot{U}}=-\nabla_{\ddot{A}} K^{\ddot{U}}+K^{\underline{I}} D_{\underline{\underline{I}}} E_{\ddot{A}}^{\ddot{U}}+E_{\ddot{A}}^{\ddot{O}} K^{\underline{I}}\left[D_{\underline{\underline{I}}}, D_{\underline{\ddot{o}}}\right\}^{\ddot{U}}+\Lambda_{l}\left[M, E_{\ddot{\tilde{A}}}^{\ddot{U}}\right]
$$

from which one follows that

$$
\triangle E_{+}^{\ddagger}=K^{\ddot{U}} D_{\underline{\ddot{U}}} E_{+}^{\ddagger}+2 i E_{+}^{+} K^{+}-E_{+}^{\ddot{U}} D_{\underline{\underline{U}}} K^{\ddagger}+\frac{1}{2} \Lambda_{l} E_{+}^{\ddagger} .
$$

It is convenient to use the s-symmetric gauge (when $E_{+}^{\ddagger}=0$ ),

$$
K^{+}=-\frac{i}{2}\left(E_{+}^{+}\right)^{-1} \nabla_{+} K^{\ddagger}
$$

and to introduce the Lorentz-invariant scalar s-field $S$ and Lorentz compensator $L$ satisfying correspondingly conditions

$$
E_{+}^{+}\left(E_{=}^{=}\right)^{1 / 2}=e^{-S} \text { and } E_{+}^{+}\left(E_{=}^{=}\right)^{-1 / 2}=e^{L} .
$$


In a Lorentz invariant theory we can always choose the gauge $L=0$; in this gauge the $\mathrm{s}-$ conformal transforms are accompanied by a corresponding compensating Lorentz transform with parameter

$$
\Lambda_{l}=\left(E_{+}^{+}\right)^{-1} \nabla_{+} K^{+}-\frac{1}{2}\left(E_{=}^{=}\right)^{-1} \nabla_{=} K^{=}=\frac{1}{2}\left(\nabla_{\ddagger} K^{\ddagger}-\nabla_{=} K^{=}\right)+\ldots
$$

The solution of constraints (54) in the s-symmetric gauge $E_{+}^{\ddagger}=L=0$ and in the linear approximation is [4]

$$
\begin{gathered}
\nabla_{+}=\left(1-\frac{S}{2}\right) D_{+}+H_{+}^{=} \partial_{=}-\left(D_{+} S+\partial_{=} H_{+}^{=}\right) M, \\
\nabla_{\ddagger}=(1-S) \partial_{\ddagger}+i\left[\frac{1}{2}\left(\partial_{=} H_{+}^{=}\right)+\left(D_{+} S\right)\right] D_{+}- \\
i\left[D_{+} H_{+}^{=}\right] \partial_{=}-\left(\partial_{\ddagger} S-i D_{+} \partial_{=} H_{+}^{=}\right) M, \\
\nabla_{=}=(1-S) \partial_{=}-\frac{i}{2}\left[\left(D_{+} H_{=}^{\ddagger}\right)\right] D_{+}+H_{=}^{\ddagger} \partial_{\ddagger}+\left(\partial_{=} S+\partial_{\ddagger} H_{=}^{\ddagger}\right) M, \\
\Sigma^{+}=\frac{i}{2}\left[D_{+}\left(\partial_{\ddagger} H_{=}^{\ddagger}+2 \partial_{=} S\right)+\partial_{=}^{2} H_{+}^{=}\right]+\ldots,
\end{gathered}
$$

where s-fields $\left(H_{+}^{=}, H_{=}^{\ddagger}\right)$ and $S$ are prepotentials of the system. These spotentials have to be used in the quantum field theory.

The linearized expression for s-field density $E^{-1}=\operatorname{Ber}\left(E \frac{\ddot{U}}{\ddot{A}}\right)$ is computed as

$$
E^{-1}=s \operatorname{det}\left(E_{\ddot{A}}^{\ddot{U}}\right)=e^{3 S / 2}\left[1+i H_{=}^{\ddagger}\left(D_{+} H_{+}^{=}+H_{+}^{=} \partial_{=} H_{+}^{=}\right)\right]^{-1} .
$$

The action for heterotic string in higher order anisotropic (1,0)-superspace accounting for the background of massless modes of locally anisotropic graviton, antisymmetric d-tensor, dilaton and gauge bosons is introduced in a manner similar to locally isotropic models [47, 55, 63, 64] but with corresponding extension to distinguished and locally adapted to $\mathrm{N}$-connection geometric objects:

$$
\begin{gathered}
I_{H S}=\frac{1}{4 \pi \alpha^{\prime}} \int d^{3} \ddot{u}^{-} E^{-1}\left\{i \nabla_{+} u^{<\alpha>} \nabla_{=} u^{<\beta>}\left[g_{<\alpha><\beta>}(u)+b_{<\alpha><\beta>}(u)\right]+\right. \\
\left.\Psi_{(-)}^{|I|}\left[\delta_{|I||J|} \nabla_{+}+A_{|I||J|}^{+}(u)\right] \Psi_{(-)}^{|J|}+\alpha^{\prime} \Phi(u) \Sigma^{+}\right\},
\end{gathered}
$$

where $A_{|I||J|}^{+}(u)=A_{|I||J|<\alpha>} \nabla_{+} u^{<\alpha>}$ is the gauge boson background, $\Phi(u)$ is the dilaton field and $\Psi_{(-)}^{|I|}$ are hetrotic fermions, $|I|,|J|, \ldots=1,2, \ldots, \mathrm{N}$. 


\section{Background D-Field Methods for $\sigma$-Models}

The background-quantum decomposition of superfields of $(1,0)$ higher order anisotropic supergravity considered in previous section can be performed in a standard manner [40] by taking into account the distinguished character of geometrical objects on locally anisotropic s-spaces; constraints (54) should be solved in terms of background-covariant derivations and quantum s-fields $\left(H_{+}^{=}, H_{=}^{\ddagger}, S\right)$, quantum s-fields $L$ and $E_{+}^{\ddagger}$ are gauged in an algebraic manner (not introducing into considerations ghosts) and note that by using quantum scale transforms we can impose gauge $S=0$ (also without Faddeev-Popov ghosts). Finally, after a background-quantum decomposition of superfields of (1.0) higher order anisotropic supergravity in the just pointed out manner we can fix the quantum gauge invariance, putting zero values for quantum fields (in absences of supergravitational and conformal anomalies and for topological trivial background configurations). In this case all $(1,0)$ supergravity fields can be considered as background ones.

The fixing of gauge symmetry as a vanishing of quantum s-fields induces the ghost action

$$
I_{F P}=\int d^{3} z^{-} E\left\{b_{=}^{\ddagger} \nabla_{+} c^{=}+b_{+}^{=} \nabla_{=} c^{\ddagger}\right\} .
$$

The aim of this section is to compute the renormalized effective action, more exactly, it anomaly part on the background of s-fields $(1,0)$ higher order anisotropic supergravity for the model defined by the action (55).

In order to integrate on quantum fields in (55) in a d-covariant manner we use background-quantum decompositions of the action with respect to normal locally adapted coordinates along autoparallels defined by Christoffel d-symbols (39). We emphasize the multiconnection character of locally anisotropic spaces; every geometric construction with a fixed $d$-connection structure (from some purposes considered as a simple or more convenient one) can be transformed, at least locally, into a another similar one for a corresponding $\mathrm{d}$-connection by using deformations of connections (40) (or (3.2) and (3.3) if we are interested in na-map deformations). Let

$$
\frac{\delta^{2} X^{<\alpha>}}{\partial s^{2}}+\left\{\frac{<\alpha>}{<\beta><\gamma>}\right\} \frac{\delta X^{<\beta>}}{\partial s} \frac{\delta X^{<\gamma>}}{\partial s}=0
$$

where $X^{<\alpha>}(s=0)=X^{<\alpha>}, X^{<\alpha>}(s=1)=X^{<\alpha>}+\pi^{<\alpha>}$ and $\pi^{<\alpha>}$ are quantum fluctuations with respect to background $X^{<\alpha>}$. Covariant quantum fields $\zeta^{<\alpha>}$ ("normal fields") are defined as

$$
\zeta^{<\alpha>}=\left.\left.\frac{\delta X^{<\alpha>}}{\partial s}\right|_{s=0} \equiv \zeta^{<\alpha>}(s)\right|_{s=0} .
$$


We shall use covariantized in a $\sigma$-model manner derivations in $(1,0)$ higher order anisotropic s-space

$$
\mathcal{D}_{\ddot{A}} \equiv\left(\mathcal{D}_{+}, \mathcal{D}_{=}\right)=\nabla_{\ddot{A}}+\Gamma_{\ddot{A}}, \Gamma_{\ddot{A}<\beta>}^{<\alpha>} \equiv\left\{\frac{\langle\alpha>}{<\gamma><\beta>}\right\} \nabla_{\ddot{A}} X^{<\gamma>},
$$

on properties of derivation $\nabla_{\ddot{A}}$ see (47) and (48), into distinguished autoparallel (in a $\sigma$-model manner) $\mathrm{d}$-covariant derivation with properties

$$
D(s) T_{<\alpha>\ldots}=\zeta^{<\beta>} \mathcal{D}_{<\beta>} T_{<\alpha>\ldots}, D(s) \zeta^{<\alpha>}(s)=0 .
$$

The derivation $\mathcal{D}_{\ddagger}$ is defined as

$$
2 i \mathcal{D}_{\ddagger} \equiv\left\{\mathcal{D}_{+}, \mathcal{D}_{+}\right\} ;
$$

we note that $\mathcal{D}_{\ddagger} \neq \nabla_{\ddagger}^{\text {cov }}$. One holds the next relations:

$$
\begin{gathered}
\mathcal{D}_{+} \zeta^{<\beta>}=\nabla_{+} \zeta^{<\beta>}+\left\{\frac{<\beta>}{<\tau><\sigma>}\right\} \nabla_{+} X^{<\sigma>} \zeta^{<\tau>}, \\
\mathcal{D}_{=} \zeta^{<\beta>}=\nabla_{=} \zeta^{<\beta>}+\left\{\frac{<\beta>}{<\tau><\sigma>}\right\} \nabla_{=} X^{<\sigma>} \zeta^{<\tau>}, \\
\mathcal{D}_{\ddagger} \zeta^{<\beta>}=\nabla_{\ddagger}^{c o v} \zeta^{<\beta>}-\frac{i}{2} R_{<\tau><\sigma><\nu>}^{<\beta>} \nabla_{+} X^{<\sigma>} \nabla_{+} X^{<\nu>} \zeta^{<\tau>}, \\
D(s) \mathcal{D}_{\ddot{A}} \zeta^{<\beta>}(s)=\zeta^{<\nu>}(s) \zeta^{<\tau>}(s) R_{<\tau><\sigma><\nu>}^{<\beta>} \nabla_{\ddot{A}} X^{<\sigma>},
\end{gathered}
$$

where

$$
\nabla_{\ddagger}^{\text {cov }} \zeta^{<\beta>}=\nabla_{\ddagger} \zeta^{<\beta>}+\left\{\frac{<\beta>}{<\tau><\sigma>}\right\} \nabla_{\ddagger} X^{<\tau>} \zeta^{<\sigma>} .
$$

For heterotic fermions the $\mathrm{d}$-covariant and gauge-covariant formalism of computation of quantum-background decomposition of action can be performed by using the prescription

$$
\Psi_{(-)}^{|I|}(s): \Psi_{(-)}^{|I|}(0)=\Psi_{(-)}^{|I|}, \Psi_{(-)}^{|I|}(s=1)=\Psi_{(-)}^{|I|}+\Delta_{(-)}^{|I|}, D_{(-)}^{2} \Psi_{-}^{|I|}(s)=0,
$$

where $\Delta_{-}^{|I|}$ are quantum fluctuations with respect to background $\Psi$; functions $\Psi_{(-)}^{|I|}(s)$ interpolate in a gauge-covariant manner with $\Psi_{(-)}^{|I|}+\Delta_{-}^{|I|}$ because of definition of the operator $D(s)$ :

$$
D(s) \Psi_{(-)}^{|I|}=\left[\delta^{|I||J|} \frac{\delta}{\partial s}+A_{<\alpha>}^{|I||J|} \frac{\delta X^{<\alpha>}}{\partial s}\right] \Psi_{(-)}^{|J|}=\frac{\delta \Psi_{(-)}^{|I|}}{\partial s}+A_{<\alpha>}^{|I||J|} \zeta^{<\alpha>} \Psi_{(-)}^{|J|} .
$$

As d-covariant and gauge-covariant quantum s-fields we use spinors

$$
\left.\chi_{(-)}^{|J|} \equiv D(s) \Psi_{(-)}^{|J|}(s)\right|_{s=0}
$$


satisfying conditions

$$
D(s) \chi_{(-)}^{|J|}(s)=0
$$

We also define the next derivation in $(1,0)$ higher order anisotropic s-superspace

$$
\left(\mathcal{D}_{+} \Psi_{(-)}\right)^{|I|} \equiv\left(\delta_{|J|}^{|I|} \nabla_{+}+A_{|J|<\alpha>}^{|I|} \nabla_{+} X^{<\alpha>}\right) \Psi_{(-)}|J|
$$

for which one holds identities

$$
\begin{gathered}
D(s) \mathcal{D}_{+} \Psi_{(-)}^{|I|}=\mathcal{D}_{+} \chi_{(-)}{ }^{|I|}+F_{|J|<\alpha><\beta>}^{|I|} \zeta^{<\alpha>} \nabla_{+} X^{<\beta>} \Psi_{(-)}{ }^{|I|}, \\
D(s) F_{|J|<\alpha><\beta>}^{|I|}=\zeta^{<\gamma>} \mathcal{D}_{<\gamma>} F_{|J|<\alpha><\beta>}^{|I|},
\end{gathered}
$$

where $\mathcal{D}_{<\gamma>}$ is both d-covariant and gauge invariant derivation and the strength $\mathrm{d}$-tensor of gauge fields is defined by using la-derivation operators,

$$
F_{|J|<\alpha><\beta>}^{|I|}=\delta_{<\alpha>} A_{|J|<\beta>}^{|I|}-\delta_{<\beta>} A_{|J|<\alpha>}^{|I|}+A_{|K|<\alpha>}^{|I|} A_{|J|<\beta>}^{|K|}-A_{|K|<\beta>}^{|I|} A_{|J|<\alpha>}^{|K|} .
$$

The action (55) consist from four groups of terms of different nature:

$$
I_{H S}=I^{(1)}+I^{(2)}+I^{(3)}+I^{(4)} .
$$

The first term

$$
I^{(1)}=-\frac{i}{4 \pi \alpha^{\prime}} \int d^{3} z^{-} E g_{<\alpha><\beta>}(X) \nabla_{+} X^{<\alpha>} \nabla_{=} X^{<\beta>}
$$

is associated to the higher order anisotropic gravitational sector and has the next background-quantum decomposition

$$
I^{(1)}[X+\pi(\zeta)]=I_{0}^{(1)}+I_{1}^{(1)}+I_{2}^{(1)}+\ldots,
$$

where

$$
I_{b}^{(1)}=\left.\frac{1}{b !} \frac{d^{b} I^{(1)}}{d s^{b}}\right|_{s=0} ; b=0,1,2, \ldots
$$

Thes terms are computed in a usual (but distinguished to the $\mathrm{N}$-connection structure) manner:

$$
\begin{gathered}
I_{1}^{(1)}=-\frac{i}{2 \pi \alpha^{\prime}} \int d^{3} z^{-} E g_{<\alpha><\beta>}\left(\mathcal{D}_{+} \zeta^{<\alpha>}\right) \nabla_{=} X^{<\beta>}, \\
I_{2}^{(1)}=-\frac{i}{4 \pi \alpha^{\prime}} \int d^{3} z^{-} E\left\{g_{<\alpha><\beta>} \mathcal{D}_{+} \zeta^{<\alpha>} \mathcal{D}_{=} \zeta^{<\beta>}+\right. \\
\left.R_{<\lambda><\sigma><\tau><\nu>} \nabla_{+} X^{<\sigma>} \nabla_{=} X^{<\nu>} \zeta^{<\lambda>} \zeta^{<\tau>}\right\}, \\
I_{3}^{(1)}=-\frac{i}{4 \pi \alpha^{\prime}} \int d^{3} z^{-} E\left\{\frac { 2 } { 3 } \left(R_{<\lambda><\sigma><\tau><\nu>} \nabla_{+} X^{<\sigma>} \mathcal{D}_{=} \zeta^{<\nu>}+\right.\right.
\end{gathered}
$$




$$
\begin{aligned}
& \left.R_{<\lambda><\sigma><\tau><\nu>} \nabla_{=} X^{<\sigma>} \mathcal{D}_{+} \zeta^{<\nu>}\right) \zeta^{<\lambda>} \zeta^{<\tau>}+ \\
& \frac{1}{3} \mathcal{D}_{<\mu>} R_{<\lambda><\sigma><\tau><\nu>} \nabla_{+} X^{<\sigma>} \nabla_{=} X^{<\nu>} \zeta^{<\mu>} \zeta^{<\lambda>} \zeta^{<\tau>}, \\
& I_{4}^{(1)}=-\frac{i}{4 \pi \alpha^{\prime}} \int d^{3} z^{-} E\left\{\frac { 1 } { 4 } \left(\mathcal{D}_{<\mu>} R_{<\lambda><\sigma><\tau><\nu>} \nabla_{+} X^{<\sigma>} \mathcal{D}_{=} \zeta^{<\nu>}+\right.\right. \\
& \left.\mathcal{D}_{<\mu>} R_{<\lambda><\sigma><\tau><\nu>} \nabla_{=} X^{<\sigma>} \mathcal{D}_{+} \zeta^{<\nu>}\right) \zeta^{<\mu>} \zeta^{<\lambda>} \zeta^{<\tau>}+ \\
& \frac{1}{3} R_{<\lambda><\sigma><\tau><\nu>} \zeta^{<\lambda>} \zeta^{<\tau>} \mathcal{D}_{+} \zeta^{<\sigma>} \mathcal{D}_{=} \zeta^{<\nu>}+ \\
& {\left[\frac{1}{3} R_{<\lambda><\sigma><\tau><\nu>} R_{<\delta><\varepsilon>} \stackrel{<\nu>}{<\gamma>}+\right.} \\
& \left.\frac{1}{12} \mathcal{D}_{<\delta>} \mathcal{D}_{<\gamma>} R_{<\lambda><\sigma><\varepsilon><\tau>}\right] \nabla_{+} X^{<\sigma>} \nabla_{=} X^{<\varepsilon>} \zeta^{<\lambda>} \zeta^{<\tau>} \zeta^{<\delta>} \zeta^{<\gamma>}, \\
& I_{5}^{(1)}=-\frac{i}{4 \pi \alpha^{\prime}} \int d^{3} z^{-} E \times \\
& \left\{\frac{1}{6}\left(\mathcal{D}_{<\mu>} R_{<\lambda><\sigma><\nu><\tau>} \mathcal{D}_{+} \zeta^{<\sigma>} \mathcal{D}_{=} \zeta^{<\nu>} \zeta^{<\mu>} \zeta^{<\lambda>} \zeta^{<\tau>}+\ldots\right\}\right. \\
& I_{6}^{(1)}=-\frac{i}{4 \pi \alpha^{\prime}} \int d^{3} z^{-} E\left\{\frac{1}{20} \mathcal{D}_{<\alpha>} \mathcal{D}_{<\mu>} R_{<\lambda><\sigma><\nu><\tau>}+\right.
\end{aligned}
$$

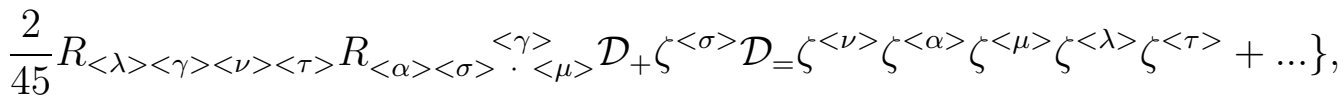

where by dots, in this section, are denoted those terms (containing multiples $(\nabla X))$ which are not important for calculation of anomalies, see section 11.

The second term in (57)

$$
I^{(2)}=-\frac{i}{4 \pi \alpha^{\prime}} \int d^{3} z^{-} E b_{<\alpha><\beta>}(X) \nabla_{+} X^{<\alpha>} \nabla_{=} X^{<\beta>}
$$

having the next background-quantum decomposition

$$
I^{(2)}[X+\pi(\zeta)]=I_{0}^{(2)}+I_{1}^{(2)}+I_{2}^{(2)}+\ldots,
$$

where

$$
I_{b}^{(2)}=\left.\frac{1}{b !} \frac{d^{b} I^{(2)}}{d s^{b}}\right|_{s=0} ; b=0,1,2, \ldots
$$

describes a Wess-Zumino-Witten like model of interactions (in a higher order anisotropic variant). The diagram vertexes depends only on intensity $H$ of antisymmetric d-tensor $b$ :

$$
H_{<\tau><\mu><\nu>}=\frac{3}{2} \delta_{[<\tau>} b_{<\mu><\nu>]}=
$$




$$
\frac{1}{2}\left(\delta_{<\tau>} b_{<\mu><\nu>}+\delta_{<\mu>} b_{<\nu><\tau>}-\delta_{<\nu>} b_{<\mu><\tau>}\right) .
$$

By straightforward calculations we find the coefficients:

$$
\begin{aligned}
& I_{1}^{(2)}=-\frac{i}{2 \pi \alpha^{\prime}} \int d^{3} z^{-} E \zeta^{<\tau>} \nabla_{+} X^{<\alpha>} \nabla_{=} X^{<\beta>} H_{<\tau><\alpha><\beta>}, \\
& I_{2}^{(2)}=-\frac{i}{4 \pi \alpha^{\prime}} \int d^{3} z^{-} E\left\{\zeta^{<\tau>} \mathcal{D}_{+} \zeta^{<\alpha>} \nabla_{=} X^{<\beta>} H_{<\tau><\alpha><\beta>}+\right. \\
& \zeta^{<\tau>} \nabla_{+} X^{<\alpha>} \mathcal{D}_{=} \zeta^{<\beta>} H_{<\tau><\alpha><\beta>}+ \\
& \left.\zeta^{<\lambda>} \zeta^{<\tau>} \nabla_{+} X^{<\alpha>} \nabla_{=} X^{<\beta>} \mathcal{D}_{<\lambda>} H_{<\tau><\alpha><\beta>}\right\}, \\
& I_{3}^{(2)}=-\frac{i}{4 \pi \alpha^{\prime}} \int d^{3} z^{-} E\left\{\frac{2}{3} \zeta^{<\tau>} \mathcal{D}_{+} \zeta^{<\mu>} \mathcal{D}_{=} \zeta^{<\nu>} H_{<\tau><\mu><\nu>}+\right. \\
& \frac{2}{3} \zeta^{<\tau>} \zeta^{<\rho>} \zeta^{<\gamma>} R_{\cdot<\gamma><\rho>[<\delta>}^{<\mu>} H_{<\nu>]<\tau><\mu>} \nabla_{+} X^{<\delta>} \nabla_{=} X^{<\nu>}+ \\
& \frac{2}{3} \zeta^{<\tau>} \zeta^{<\lambda>}\left(\mathcal{D}_{+} \zeta^{<\mu>} \nabla_{=} X^{<\nu>}+\mathcal{D}_{=} \zeta^{<\nu>} \nabla_{+} X^{<\mu>}\right) \mathcal{D}_{<\lambda>} H_{<\tau><\mu><\nu>}+ \\
& \frac{1}{3} \zeta^{<\sigma>} \zeta^{<\lambda>} \zeta^{<\tau>} \nabla_{+} X^{<\alpha>} \nabla_{=} X^{<\beta>} \mathcal{D}_{<\sigma>} \mathcal{D}_{<\lambda>} H_{<\tau><\alpha><\beta>}, \\
& I_{4}^{(2)}=-\frac{i}{4 \pi \alpha^{\prime}} \int d^{3} z^{-} E\left\{\frac{1}{2} \zeta^{<\tau>} \zeta^{<\lambda>} \mathcal{D}_{+} \zeta^{<\mu>} \mathcal{D}_{=} \zeta^{<\nu>} H_{<\tau><\mu><\nu>}+\right. \\
& \frac{1}{6} \zeta^{<\lambda>} \zeta^{<\tau>} \zeta^{<\rho>} \zeta^{<\gamma>} \nabla_{+} X^{<\delta>} \nabla_{=} X^{<\nu>} \mathcal{D}_{<\lambda>}\left(R_{\cdot<\gamma><\rho>[<\delta>}^{<\mu>} H_{<\nu>]<\tau><\mu>}\right)+ \\
& \frac{2}{3} \zeta^{<\tau>} \zeta^{<\rho>} \zeta^{<\gamma>} R_{.<\gamma><\rho>[<\delta>}^{<\mu>} H_{<\nu>]<\tau><\mu>} \times\left(\nabla_{+} X^{<\delta>} \mathcal{D}_{=} X^{<\nu>}+\right. \\
& \left.\mathcal{D}_{+} X^{<\nu>} \nabla_{=} X^{<\delta>}\right)+\zeta^{<\lambda>} \zeta^{<\tau>} \zeta^{<\alpha>} \zeta^{<\beta>} \nabla_{+} X^{<\nu>} \nabla_{=} X^{<\rho>} \times \\
& \left(\frac{1}{3} \mathcal{D}_{<\lambda>} H_{<\tau><\mu>[<\rho>} R_{<\nu><\alpha>} \stackrel{<\mu>}{\cdot<\beta>}-\frac{1}{12} \mathcal{D}_{<\alpha>} \mathcal{D}_{<\beta>} \mathcal{D}_{<\gamma>} H_{<\tau><\nu><\rho>}\right)+ \\
& \frac{1}{4} \zeta^{<\tau>} \zeta^{<\lambda>} \zeta^{<\gamma>}\left(\mathcal{D}_{+} \zeta^{<\mu>} \nabla_{=} X^{<\nu>}+\right. \\
& \left.\left.\mathcal{D}=\zeta^{<\nu>} \nabla_{+} X^{<\mu>}\right) \mathcal{D}_{<\gamma>} \mathcal{D}_{<\lambda>} H_{<\tau><\mu><\nu>}\right\}, \\
& I_{5}^{(2)}=-\frac{i}{4 \pi \alpha^{\prime}} \int d^{3} z^{-} E\left\{\frac{1}{2} \zeta^{<\tau>} \zeta^{<\lambda>} \zeta^{<\gamma>} \mathcal{D}_{+} \zeta^{<\mu>} \mathcal{D}_{=} \zeta^{<\nu>} \times\right. \\
& {\left[\frac{1}{5} \mathcal{D}_{<\gamma>} \mathcal{D}_{<\lambda>} H_{<\tau><\mu><\nu>}+\frac{2}{15} R_{\cdot<\gamma><\lambda>[<\mu>}^{<\rho>} H_{<\nu>]<\tau><\rho>}\right\}+\ldots,} \\
& I_{6}^{(2)}=-\frac{i}{4 \pi \alpha^{\prime}} \int d^{3} z^{-} E \zeta^{<\tau>} \zeta^{<\lambda>} \zeta^{<\alpha>} \zeta^{<\beta>} \mathcal{D}_{+} \zeta^{<\rho>} \mathcal{D}_{=} \zeta^{<\nu>} \times
\end{aligned}
$$




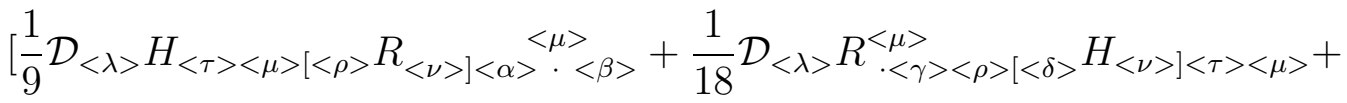

$$
\begin{aligned}
& \left.\frac{1}{18} \mathcal{D}_{<\alpha>} \mathcal{D}_{<\beta>} \mathcal{D}_{<\lambda>} H_{<\tau><\rho><\nu>}\right]+\ldots,
\end{aligned}
$$

where by dots are denoted terms not being important for calculation of anomalies and operations of symmetrization ( ) and antisymmetrization are taken without coefficients.

The third term in (57)

$$
I^{(3)}=I_{0}^{(3)}+I_{1}^{(3)}+I_{2}^{(3)}+\ldots+I_{s}^{(3)}+\ldots
$$

is of Fradkin-Tseitlin dilaton type with coefficients

$$
\begin{gathered}
I_{0}^{(3)}=-\frac{1}{4 \pi \alpha^{\prime}} \int d^{3} z^{-} E \alpha^{\prime} \Sigma^{+} \Phi \\
I_{1}^{(3)}=-\frac{1}{4 \pi \alpha^{\prime}} \int d^{3} z^{-} E \alpha^{\prime} \Sigma^{+} \zeta^{<\alpha>} \delta_{<\alpha>} \Phi \\
I_{2}^{(3)}=-\frac{1}{4 \pi \alpha^{\prime}} \int d^{3} z^{-} E \alpha^{\prime} \Sigma^{+} \frac{1}{2 !} \zeta^{<\beta>} \zeta^{<\alpha>} \mathcal{D}_{<\alpha>} \mathcal{D}_{<\beta>} \Phi, \ldots, \\
I_{s}^{(3)}=-\frac{1}{4 \pi \alpha^{\prime}} \int d^{3} z^{-} E \alpha^{\prime} \Sigma^{+} \frac{1}{s !} \zeta^{<\alpha_{1}>} \ldots \zeta^{<\alpha_{s}>} \mathcal{D}_{<\alpha_{1}>\ldots} \mathcal{D}_{<\alpha_{s}>} \Phi .
\end{gathered}
$$

The forth term in $(57)$

$$
I^{(4)}=-\frac{1}{4 \pi \alpha^{\prime}} \int d^{3} z^{-} E \Psi_{(-)}{ }^{|I|} \mathcal{D}_{+} \Psi_{(-)}|I|
$$

has the next background-quantum decomposition

$$
I^{(4)}[\Psi+\Delta(\chi), X+\pi(\zeta)]=I_{0}^{(4)}+I_{1}^{(4)}+I_{2}^{(4)}+\ldots,
$$

with coefficients

$$
\begin{aligned}
& I_{1}^{(4)}=-\frac{1}{4 \pi \alpha^{\prime}} \int d^{3} z^{-} E\left\{\chi_{(-)}^{|I|} \mathcal{D}_{+} \chi_{(-)}{ }^{|I|}+\Psi_{(-)}^{|I|} \mathcal{D}_{+} \Psi_{(-)}{ }^{|I|}+\right. \\
& \left.\Psi_{(-)}^{|I|} F_{|I||J|<\mu><\nu>} \zeta^{<\mu>} \nabla_{+} X^{<\nu>} \Psi_{(-)}^{|J|}\right\}, \\
& I_{2}^{(4)}=-\frac{1}{4 \pi \alpha^{\prime}} \int d^{3} z^{-} E\left\{\chi_{(-)}{ }^{|I|} \mathcal{D}_{+} \chi_{(-)}{ }^{|I|}+\right. \\
& 2 \chi_{(-)}^{|I|} F_{|I||J|<\mu><\nu>} \zeta^{<\mu>} \nabla_{+} X^{<\nu>} \Psi_{(-)}^{|J|} \\
& \frac{1}{2} \zeta^{<\nu>} \zeta^{<\mu>} \Psi_{(-)}^{|I|} \Psi_{(-)}{ }^{|J|} \mathcal{D}_{<\lambda>} F_{|I||J|<\mu><\nu>} \nabla_{+} X^{<\nu>}-
\end{aligned}
$$




$$
\begin{aligned}
& \left.\frac{1}{2} \Psi_{(-)}^{|I|} \Psi_{(-)}{ }^{|J|} F_{|I||J|<\mu><\nu>} \zeta^{<\mu>} \mathcal{D}_{+} \zeta^{<\nu>}\right\}, \\
& I_{3}^{(4)}=-\frac{1}{4 \pi \alpha^{\prime}} \int d^{3} z^{-} E\left\{\chi_{(-)}^{|I|} F_{|I||J|<\mu><\nu>} \zeta^{<\mu>} \nabla_{+} X^{<\nu>} \chi_{(-)}{ }^{|J|}+\right. \\
& \zeta^{<\lambda>} \zeta^{<\mu>} \chi_{(-)}{ }^{|I|} \mathcal{D}_{<\lambda>} F_{|I||J|<\mu><\nu>} \nabla_{+} X^{<\nu>} \Psi_{(-)}|J|+ \\
& \chi_{(-)}{ }^{|I|} F_{|I||J|<\mu><\nu>} \zeta^{<\mu>} \mathcal{D}_{+} \zeta^{<\nu>} \Psi_{(-)}|J|- \\
& \frac{1}{3} \zeta^{<\lambda>} \zeta^{<\nu>} \Psi_{(-)}{ }^{|I|} \mathcal{D}_{<\lambda>} F_{|I||J|<\mu><\nu>} \Psi_{(-)}|J| \mathcal{D}_{+} \zeta^{<\mu>}- \\
& \frac{1}{6} \zeta^{<\lambda>} \zeta^{<\tau>} \zeta^{<\mu>} \Psi_{(-)}{ }^{|I|} \Psi_{(-)}{ }^{|J|} \nabla_{+} X^{<\nu>} \times \\
& \left(\mathcal{D}_{<\tau>} \mathcal{D}_{<\lambda>} F_{|I||J|<\mu><\nu>}-R_{<\lambda>]<\nu>} \stackrel{<\gamma><\tau>}{ } F_{|I||J|<\mu><\nu>}\right), \\
& I_{4}^{(4)}=-\frac{1}{4 \pi \alpha^{\prime}} \int d^{3} z^{-} E\left\{\frac{1}{2} \chi_{(-)}^{|I|} F_{|I||J|<\mu><\nu>} \chi_{(-)}{ }^{|J|} \zeta^{<\nu>} \mathcal{D}_{+} \zeta^{<\mu>}-\right. \\
& \frac{1}{2} \zeta^{<\lambda>} \zeta^{<\mu>} \chi_{(-)}{ }^{|I|}\left(\mathcal{D}_{<\lambda>} F_{|I||J|<\mu><\nu>}\right) \chi_{(-)}{ }^{|J|} \nabla_{+} X^{<\nu>}+ \\
& \frac{2}{3} \zeta^{<\lambda>} \zeta^{<\mu>} \chi_{(-)}{ }^{|I|}\left(\mathcal{D}_{<\lambda>} F_{|I||J|<\mu><\nu>}\right) \Psi_{(-)}{ }^{|J|} \mathcal{D}_{+} \zeta^{<\nu>}+\frac{1}{3} \zeta^{<\gamma>} \zeta^{<\lambda>} \zeta^{<\mu>} \chi_{(-)}{ }^{|I|} \times \\
& \left(\mathcal{D}_{<\gamma>} \mathcal{D}_{<\lambda>} F_{|I||J|<\mu><\nu>}+R_{<\gamma><\nu>} \stackrel{<\rho>}{<\lambda>} F_{|I||J|<\mu><\rho>}\right) \nabla_{+} X^{<\nu>} \Psi_{(-)}|J|- \\
& \frac{1}{24} \zeta^{<\lambda>} \zeta^{<\mu>} \zeta^{<\gamma>} \Psi_{(-)}{ }^{|I|} \Psi_{(-)}|J|\left(3 \mathcal{D}_{<\mu>} \mathcal{D}_{<\lambda>} F_{|I||J|<\gamma><\nu>}+\right. \\
& \left.R_{<\lambda><\nu>} \stackrel{<\rho>}{<\gamma>} F_{|I||J|<\tau><\rho>}\right) \mathcal{D}_{+} \zeta^{<\nu>}-\frac{1}{24} \zeta^{<\tau>} \zeta^{<\lambda>} \zeta^{<\mu>} \zeta^{<\gamma>} \Psi_{(-)}{ }^{|I|} \Psi_{(-)}{ }^{|J|} \times \\
& {\left[\mathcal{D}_{<\gamma>} \mathcal{D}_{<\tau>} \mathcal{D}_{<\lambda>} F_{|I||J|<\mu><\nu>}+\left(\mathcal{D}_{<\gamma>} R_{<\lambda><\nu>} \stackrel{<\rho>}{{ }^{\prime}<\mu>}\right) F_{|I||J|<\tau><\rho>}+\right.} \\
& \left.\left.3\left(\mathcal{D}_{<\gamma>} F_{|I||J|<\tau><\rho>}\right) R_{<\lambda><\nu>} \stackrel{<\rho>}{\cdot<\mu>}\right] \nabla_{+} X^{<\nu>}\right\}, \\
& I_{5}^{(4)}=-\frac{1}{4 \pi \alpha^{\prime}} \int d^{3} z^{-} E\left\{-\frac{1}{3} \zeta^{<\mu>} \zeta^{<\lambda>} \chi_{(-)}|I|\right. \\
& \left.\left(\mathcal{D}_{<\lambda>} F_{|I||J|<\mu><\nu>}\right) \chi_{(-)}{ }^{|J|} \mathcal{D}_{+} \zeta^{<\mu>}+\ldots\right\} \\
& I_{6}^{(4)}=-\frac{1}{4 \pi \alpha^{\prime}} \int d^{3} z^{-} E\left\{-\frac{1}{24} \zeta^{<\gamma>} \zeta^{<\mu>} \zeta^{<\lambda>} \chi_{(-)}^{|I|} \chi_{(-)}{ }^{|J|} \times\right. \\
& \left.\left(3 \mathcal{D}_{<\gamma>} \mathcal{D}_{<\lambda>} F_{|I||J|<\mu><\nu>}+F_{|I||J|<\gamma><\rho>} R_{<\lambda><\nu>} \stackrel{<\rho>}{\cdot<\mu>}\right) \mathcal{D}_{+} \zeta^{<\nu>}\right\}+\ldots
\end{aligned}
$$

The kinetic terms for quantum fields $\zeta^{<\mu>}$ and $\chi_{(-)}|J|$ in the decompositions (2.14) and (2.15) define the propagators $\left(2 \pi \alpha^{\prime}=1\right)$

$$
<\zeta^{<\mu>}(\ddot{u}) \zeta^{<\nu>}\left(\ddot{u}^{\prime}\right)>=g^{<\mu><\nu>} \frac{D_{+}}{\square} \delta_{(-)}^{3}\left(\ddot{u}, \ddot{u}^{\prime}\right)=
$$




$$
\begin{aligned}
& g^{<\mu><\nu>} \frac{1}{(2 \pi)^{2}} \int d^{d_{2}} p \frac{1}{\left(-p^{2}\right)} D_{+}\left[e^{i p\left(z-z^{\prime}\right)} \delta_{(-)}\left(\theta-\theta^{\prime}\right)\right] \\
& <\chi_{(-)}^{|I|}(\ddot{u}) \chi_{(-)}^{|J|}\left(\ddot{u}^{\prime}\right)>=i \delta^{|I||J|} \frac{\partial_{=} D_{+}}{\square} \delta_{(-)}^{3}\left(\ddot{u}, \ddot{u}^{\prime}\right)= \\
& \frac{\delta^{|I||J|}}{(2 \pi)^{2}} \int d^{d_{2}} p \frac{p_{=}}{p^{2}} D_{+}\left[e^{i p\left(z-z^{\prime}\right)} \delta_{(-)}^{3}\left(\ddot{u}, \ddot{u}^{\prime}\right)\right] .
\end{aligned}
$$

Finally, we remark that background-quantum decompositions of the action (55) for heterotic string define the Feynman rules (vertixes and propagators) for the corresponding generalization of the two-dimensional sigma model which are basic for a perturbation quantum formalism in higher order anisotropic spaces.

\section{Green-Schwarz Action in DVS-Bundles}

The Green-Scwarz covariant action ( GS-action ) for superstrings can be considered as a two dimensional $\sigma$-model with Wess-Zumino-Witten term and flat, dimension $d=10$, s-space as the tangent space [43, 49, 50, 73]. The GS-action was generalized for the curved background $\mathrm{N}=1, d=10$ of the superspace under the condition that motion equations hold [127] and under similar conditions for $\mathrm{N}=2, d=10$ supergravity 45 .

The GS-action in dimensions $d=3,4,6,10$ can be represented as

$$
\begin{gathered}
I=\frac{1}{2} \int d^{2} z \sqrt{-\gamma} \gamma^{\dddot{e}} \partial_{\ddot{e}} u^{<\alpha>} \partial_{\ddot{\imath}} u^{<\beta>}\left(l_{<\underline{\alpha}>}^{<\underline{\alpha}>} l_{<\bar{\beta}>}^{<>}\right) \widehat{\eta}_{<\underline{\alpha}><\underline{\beta}>}+ \\
\frac{1}{2} \int d^{2} z \varepsilon^{\dddot{e} i} \partial_{\ddot{e}} u^{<\alpha>} \partial_{\ddot{i}} u^{<\beta>} B_{<\alpha><\beta>}
\end{gathered}
$$

by using of the flat vielbein $l_{<\alpha}^{<\alpha>}$ and 2 -form

$$
B=\frac{1}{2} \delta u^{<\alpha>} \Lambda \delta u^{<\beta>} B_{<\alpha><\beta>}
$$

in the flat $d=10 \mathrm{~s}-$ space with coordinates $u^{<\alpha>}$. An important role in formulation of the GS-action plays the fact that 3 -form $H=d B$ is closed, $d H=0$. Because $H$ is s-invariant the 2 -form $B$ changes on complete derivation under s-transforms

$$
\delta^{\star} H=0, \delta^{\star} d B=d \delta^{\star} B=0, \delta^{\star} B=d \Lambda,
$$

where $\delta^{\star}$ is dual to $d$, which ensures the $\mathrm{s}$-invariance of the GS-action.

We generalize the action (60) for higher order anisotropic s-spaces by chang-

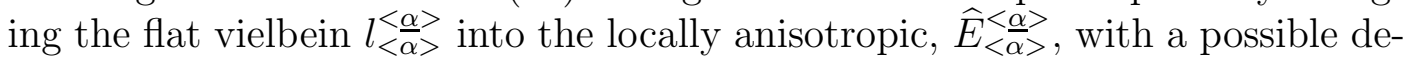
pendence of the Lagrangian on scalar fields (see [87] for locally isotropic spaces),

$$
I=\frac{1}{2} \int d^{2} z\left[\sqrt{-\gamma} \gamma^{\dddot{e} i} \partial_{\ddot{e}} u^{<\alpha>} \partial_{i} u^{<\beta>}\left(E_{<\underline{\alpha}>}^{<\alpha} E_{<\bar{\beta}>}^{<\beta}\right) \widehat{\eta}_{\langle\underline{\alpha}><\underline{\beta}>}+\right.
$$




$$
\begin{gathered}
\varepsilon^{\dddot{e} \ddot{\imath}} \partial_{\ddot{e}} u^{<\alpha>} \partial_{\ddot{\imath}} u^{<\beta>} E_{<\underline{\alpha}>}^{<\alpha>} E_{<\bar{\beta}>}^{<\beta} B_{<\underline{\alpha}><\underline{\beta}>}+ \\
\left.V V_{\underline{\underline{e}}}^{\ddot{\Psi}} \bar{\Psi}^{|\underline{I}|} \gamma^{\ddot{\underline{e}}}\left(\partial_{\ddot{e}} \delta_{|\underline{I}||\underline{J}|}+E_{\ddot{e}}^{<\alpha>} A_{|\underline{I}||\underline{J}|<\alpha>}\right) \Psi^{|\underline{J}|}\right],
\end{gathered}
$$

where $P$ is a scalar function, $\gamma_{\ddot{e} i}=V_{\ddot{e}} V_{\ddot{i}}^{\underline{e}} \gamma_{\underline{e ̈}}, V=\operatorname{det}\left(V_{\ddot{e}}^{\ddot{e}}\right), u^{<\alpha>}$ are coordinates of the higher order anisotropic s-space and $\Psi^{|\underline{J}|}$ are two dimensional (heterotic) MW-fermions in the fundamental representation of the interior symmetry group Gr.

As background s-fields we shall consider

$$
\begin{gathered}
E^{<\underline{\alpha}>}=d \widehat{u}^{<\beta>} E_{<\bar{\beta}>}^{<\underline{\alpha}>}(z), B=\frac{1}{2} E^{<\underline{\alpha}>} E^{<\underline{\beta}>} B_{<\underline{\alpha}><\underline{\beta}>}(z), \\
A_{|\underline{I}||\underline{J}|}=A_{|\underline{I}||\underline{J}|<\underline{\alpha}>} E^{<\underline{\alpha}>},
\end{gathered}
$$

where

$$
E_{\ddot{a}}^{<\underline{\alpha}>} \equiv \partial_{\ddot{a}} u^{<\alpha>} E_{<\underline{\alpha}>}^{<\underline{\alpha}>}=\left(\widehat{E}_{\ddot{a}}^{<\underline{\alpha}>}, E_{\ddot{a}}^{<\underline{\alpha}>}=E_{\ddot{a}}^{\ddot{a}}\right) .
$$

S-fields $A_{|\underline{I}||\underline{J}|}$ belong to the adjoint representation of the interior symmetry group $G r$.

The action (61) is invariant under transforms $\left(\triangle E^{<\underline{\alpha}>} \equiv \triangle u^{<\alpha>} E_{<\underline{\alpha}>}^{<-\alpha}\right)$ :

$$
\begin{aligned}
& \triangle \widehat{E}^{<\underline{\alpha}>}=0, \triangle E^{\ddot{e}}=2\left(\Gamma_{<\underline{\alpha}>}\right)^{\dddot{e} o} \widehat{E}_{\ddot{e}}^{<\underline{\alpha}>} V_{\underline{\underline{e}}}^{\ddot{e}} k_{\ddot{o}}^{\ddot{o}},
\end{aligned}
$$

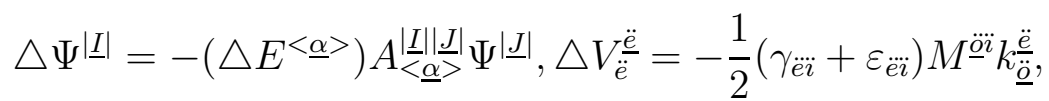

where $\varepsilon_{\ddot{e} i}$ is defined as a two-dimensional tensor, parameter $k \stackrel{\ddot{e}}{<\alpha>}$ is anti-selfdual as a two-vector, juggling of indices of $d$-dimensional Dirac matrices is realized by using the $d$-dimensional matrix of charge conjugation and, for simplicity, we can consider matrices $\left(\Gamma_{<\underline{\alpha}>}\right)^{\ddot{e} o ̈}$ as symmetric; we shall define below the value $M^{<\alpha>i}$.

The variation of action (61) under transforms (62) can be written as

$$
\begin{gathered}
\triangle I=\int d^{2} z \frac{1}{2}\left[e^{P} \triangle\left(V \gamma^{\dddot{e} i}\right) \widehat{E}_{\ddot{e}}^{<\underline{\alpha}>} \widehat{E}_{\ddot{i}}^{<\underline{\beta}>} \eta_{<\underline{\alpha}><\underline{\beta}>}+\right. \\
\triangle E^{\ddot{o}}\left(e^{P}\left(V \gamma^{\dddot{e} i}\right) \widehat{E}_{\ddot{e}}^{<\underline{\alpha}>} \widehat{E}_{\ddot{i}}^{<\underline{\beta}>} \eta_{<\underline{\alpha}><\underline{\beta}>} D_{\ddot{o}} P-2 e^{P} \triangle\left(V \gamma^{\dddot{e} i}\right) E_{\ddot{e}}^{<\underline{\alpha}>} E_{\ddot{i}}^{<\underline{\beta}>} T_{<\underline{\alpha}>\ddot{o}<\underline{\beta}>}+\right. \\
\left.\varepsilon^{\dddot{e} i} E_{\ddot{e}}^{<\underline{\alpha}>} E_{\ddot{i}}^{<\underline{\beta}>} H_{<\underline{\alpha}><\underline{\beta}>\ddot{o}}-V \bar{\Psi}^{|\underline{I}|} \gamma^{\ddot{e}} \Psi \mid \underline{J \mid} E_{\ddot{e}}^{<\underline{\alpha}>} F_{<\underline{\alpha}>\ddot{o}|\underline{I}||\underline{J}|}\right)+\triangle\left(V V_{\ddot{e}}^{\ddot{e})} \bar{\Psi}^{|\underline{I}|} \gamma^{\ddot{e}}\left(\mathcal{D}_{\ddot{e}} \Psi\right)^{|\underline{J}|},\right.
\end{gathered}
$$

where $\mathcal{D}_{\ddot{e}}$ is the $G r$-covariant derivation and the torsion 2 -form $T^{<\underline{\alpha}>}$, the strength 3 -form $H$ and the supersymmetric Yang-Mills strength 2-form $F^{\mid \underline{I}}|\underline{\underline{J}}|$ are respectively defined by relations

$$
T^{<\underline{\alpha}>}=d E^{<\underline{\alpha}>}+E^{<\underline{\beta}>} \Omega_{<\underline{\beta}>}^{<\underline{\alpha}>}=\frac{1}{2} E^{<\underline{\beta}>} E^{<\underline{\gamma}>} T_{<\underline{\beta}><\underline{\gamma}>}^{<\underline{\alpha}>},
$$




$$
\begin{gathered}
H=d B=\frac{1}{6} E^{<\underline{\gamma}>} E^{<\underline{\beta}>} E^{<\underline{\alpha}>} H_{<\underline{\alpha}><\underline{\beta}><\underline{\gamma}>,} \\
F^{|\underline{I}||\underline{J}|}=d A^{|\underline{I}||\underline{J}|}+A^{|\underline{I}||\underline{K}|} A^{|\underline{K}||\underline{J}|}=\frac{1}{2} E^{<\underline{\beta}>} E^{<\underline{\gamma}>} F_{<\underline{\beta}>\underline{|\underline{I}||\underline{J}|} .} .
\end{gathered}
$$

The variation of action (63) under transforms (63) vanishes if and only if there are satisfied the next conditions:

1) 3-form $H$ is closed under condition

$$
\left(\Gamma^{<\underline{\alpha}>}\right)_{\dddot{e} i}\left(\Gamma_{<\underline{\alpha}>}\right)_{\dddot{o} \ddot{u}}+\left(\Gamma^{<\underline{\alpha}>}\right)_{\dddot{\imath} \ddot{o}}\left(\Gamma_{<\underline{\alpha}>}\right)_{\ddot{e} \ddot{u}}+\left(\Gamma^{<\underline{\alpha}>}\right)_{\dddot{o} \ddot{e}}\left(\Gamma_{<\underline{\alpha}>}\right)_{\dddot{\imath} \ddot{u}}=0,
$$

which holds for dimensions $d=3,4,6,10$;

2) there are imposed constraints

$$
\begin{aligned}
& \widehat{T}_{\ddot{e} \ddot{i}}^{<\underline{\alpha}>}=-i\left(\widehat{\Gamma}^{<\underline{\alpha}>}\right)_{\dddot{e} i \ddot{i}}, \widehat{\eta}_{<\gamma>(<\alpha>} \widehat{T}_{<\beta>) \ddot{a}}^{<\gamma>}=\widehat{\eta}_{<\alpha><\beta>} B_{\ddot{a}}, F_{\ddot{e} i}^{|\underline{I}||\underline{J}|}=0, \\
& F_{<\underline{\alpha}>i}^{|\underline{I}||\underline{J}|}=\left(\widehat{\Gamma}_{<\underline{\alpha}>}\right)_{\ddot{e} i} w^{\ddot{e}|\underline{I}||\underline{J}|}, H_{\dddot{e} \dddot{\imath} \ddot{O}}=0, H_{\dddot{e ̈}<\underline{\alpha}>}=-i e^{P}\left(\widehat{\Gamma}_{<\underline{\alpha}>}\right)_{\dddot{e} i}, \\
& \widehat{H}_{\ddot{u}<\alpha><\beta>}=2 e^{P}\left(\Gamma_{<\underline{\alpha}>}\right)_{\ddot{o}}^{\ddot{e}}\left(\Gamma_{<\underline{\beta}>}\right)_{\ddot{u}}^{\ddot{o}} H_{\ddot{e}} ;
\end{aligned}
$$

3) The coefficient $M^{\underline{\underline{o} i}}$ from (62) is taken in the form

$$
M^{\dddot{\dddot{o} i}}=4 i E^{\underline{\underline{\underline{o}}}}-4 \widehat{E}_{<\underline{\alpha}>}^{\ddot{i}}\left(\widehat{\Gamma}^{<\underline{\alpha}>}\right) \underline{\ddot{o} \ddot{u}} H_{\underline{\ddot{u}}}-\Psi^{|\underline{I}|} \gamma^{\ddot{\imath}} \Psi^{|\underline{J}|} w_{|\underline{\underline{I}}||\underline{J}|} e^{-P},
$$

where

$$
D_{\underline{\ddot{u}}} P+2 H_{\underline{\ddot{u}}}-2 B_{\underline{\ddot{u}}}=0 ;
$$

4) The last term in (63) vanishes because of conditions of chirality,

$$
\Psi^{|\underline{I}|}=-\gamma_{5} \Psi^{|\underline{\mid}|} .
$$

In the locally isotropic s-gravity it is known 41, 82, 10 that s-field equations of type (64) are compatible with Bianchi identities and can be interpreted as standard constraints defining supergravity in the superspace. Considering locally adapted to $\mathrm{N}$-connections geometric objects end equations (64) we obtain a variant of higher order anisotropic supergravity (see [106, 110, 111, 112] for details on locally anisotropic supergravity) which for dimensions $d=n+m=10$ contain, distinguished by the $\mathrm{N}$-connection structure, motion equations of $\mathrm{N}=1$ of higher order anisotropic supergravity and super-Yang-Mills matter.

The above presented constructions can be generalized in order to obtain a variant of higher order anisotropic $\mathrm{N}=2, d=10$ supergravity from so-called IIBsuperstrings [45] (which, in our case, will be modified to be locally anisotropic). To formulate the model we use a locally adapted s-vielbein 1 -form $E^{<\underline{\alpha}>}=$ 


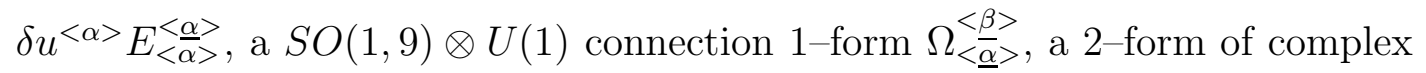
potential $A$ and one real 4 -form $B$. Strengths are defined in a standard manner:

$$
\begin{gathered}
T^{<\underline{\alpha}>}=D E^{<\underline{\alpha}>}=\delta E^{<\underline{\alpha}>}+E^{<\underline{\beta}>} \Omega_{<\underline{\beta}>}^{<\underline{\alpha}>}, \\
R_{<\underline{\beta}>}^{<\underline{\alpha}>}=\delta \Omega_{<\underline{\alpha}>}^{<\underline{\beta}>}+\Omega_{<\underline{\alpha}>}^{<\underline{\alpha}>} \Omega_{<\underline{\gamma}>}^{<\underline{\beta}>}, \\
F=\delta A, G=\delta B+A \bar{F}-\bar{A} F .
\end{gathered}
$$

On the mass shell (on locally anisotropic spaces we shall consider distinguished metrics) ds-tensors (65) are expressed in terms of one scalar s-field $V \in S U(1,1):$

$$
V=\left(\begin{array}{cc}
q & s \\
\bar{u} & \bar{v}
\end{array}\right), q \bar{q}-s \bar{s}=1 .
$$

Excluding a scalar by using the local U(1)-invariance we can use the first components of complex s-fields $(q, s)$ as physical scalar fields of the theory.

The constraints defining IIB supergravity in $d=n+m=10$ higher order anisotropic s-space contain equations (on every anisotropic "shell", in locally adapted frames, they generalize constraints of IIB supergavity [52]):

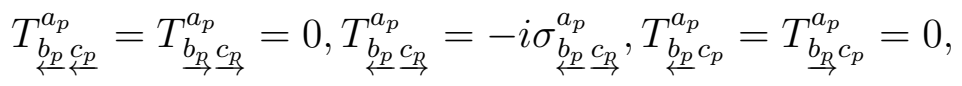

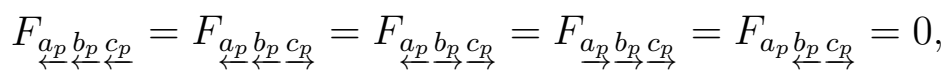

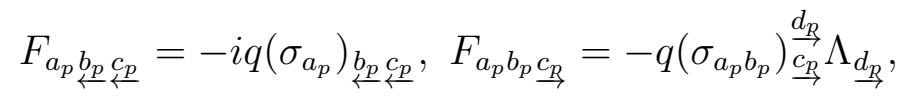

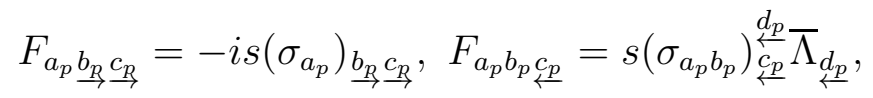

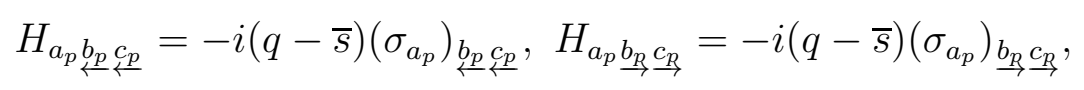

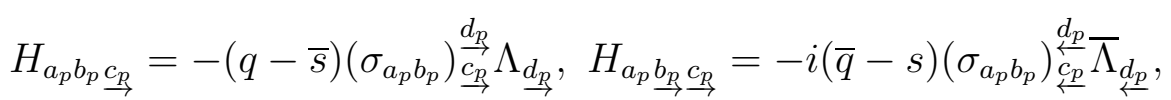

where

$$
\left(\sigma_{a_{p}} \sigma_{b_{p}}\right) \stackrel{d_{p}}{\stackrel{c_{p}}{\rightarrow}}=\left(\sigma_{a_{p} b_{p}}\right) \stackrel{d_{p}}{\stackrel{c_{p}}{\longrightarrow}}+\eta_{a_{p} b_{p}} \delta_{\underline{c_{p}}}^{\stackrel{d_{p}}{\rightarrow}}
$$

the same formula holds for " $\leftarrow$ "-underlined spinors, spinor $\Lambda_{\underline{d_{p}}}$ will be used below for fixing of $U(1)$ gauge and 3-form $H \equiv F+\bar{F}=\delta \widetilde{B}$ is real and closed (this condition is crucial in the construction of the GS-action on the background of IIB-supergravity, with respect to usual isotropic string model see [45]). 
The action (60) can be generalized for $\mathrm{N}=2$ higher order anisotropic $\mathrm{s}-$-spaces in this manner:

$$
I_{S}=\int d^{2} z\left\{\sqrt{-\gamma} \gamma^{\dddot{u}} P(q, s) \widehat{E}_{\ddot{i}}^{<\underline{\alpha}>} \widehat{E}_{\ddot{u}}^{<\underline{\beta}>} \widehat{\eta}_{\langle\underline{\alpha}><\underline{\beta}>}+\frac{1}{2} \varepsilon^{\dddot{\imath} \ddot{u}} E_{i}^{<\alpha>} E_{\ddot{u}}^{<\beta>} \widetilde{B}_{\langle\alpha><\beta>},\right.
$$

where $P(q, s)$ is a function of scalar fields $q$ and $s$, and

$$
E_{\ddot{i}}^{<\underline{\alpha}>} \equiv \partial_{i} u^{<\alpha>} E_{<\underline{\alpha}>}^{<\underline{\alpha}>}=\left(\widehat{E}_{i}^{<\underline{\alpha}>}, \ldots, E_{i}^{\stackrel{a_{p}}{<}}, \ldots, \bar{E}_{\ddot{i}}^{\underline{b_{p}}}, \ldots\right) .
$$

The variation of the Lagrangian in (67) under respective $\mathrm{k}$-transforms of type (62) can be written as

$$
\begin{gathered}
\triangle L=\sqrt{-\gamma} \gamma^{\dddot{u}} P E_{\ddot{i}}^{<\gamma>} \triangle E^{<\beta>} T_{<\bar{\beta}><\gamma>}^{<\delta>} E_{\ddot{u}}^{<\underline{\beta}>} \widehat{\eta}_{<\underline{\delta}><\underline{\beta}>}+ \\
\frac{1}{2} \varepsilon^{\dddot{\imath} \ddot{u}} E_{\ddot{i}}^{<\gamma>} E_{\ddot{u}}^{<\beta>} \triangle E^{<\tau>} H_{<\tau><\beta><\gamma>}+ \\
\frac{1}{2}\left[\triangle\left(\sqrt{-\gamma} \gamma^{\dddot{\imath} \ddot{u}} P\right)+\sqrt{-\gamma} \gamma^{\dddot{\imath} \ddot{u}} \triangle P\right] \widehat{E}_{\ddot{i}}^{<\underline{\alpha}>} \widehat{E}_{\ddot{u}}^{<\underline{\beta}>} \widehat{\eta}_{\langle\underline{\alpha}><\underline{\beta}>>} .
\end{gathered}
$$

Taking into account constraints (66) we can express variation (69) as

$$
\begin{aligned}
& \triangle L=\left(\left\{-i\left(\varepsilon^{\dddot{\imath} \ddot{u}} \gamma^{\dddot{e} a}(q-\bar{s})+\gamma^{\dddot{\imath} \ddot{u}} \varepsilon^{\dddot{e} \ddot{a}} P\right) \widehat{E}_{\ddot{e}}^{<\gamma>} \widehat{E}_{\ddot{i}}^{<\beta>}\left(\sigma_{<\beta>} \sigma_{<\gamma>}\right)\right)_{\underset{\tau}{\tau}}^{\frac{\delta}{\tau}} E_{\ddot{u}}^{\frac{\tau}{\tau}} k_{\ddot{a} \_}-\right. \\
& \left.i\left[(-\gamma)^{-1 / 2} \varepsilon^{\dddot{\imath} \ddot{u}} \varepsilon^{\dddot{e} a}(\bar{q}-s)+\sqrt{-\gamma} \gamma^{\dddot{\imath u}} \gamma^{\dddot{e} a} P\right] \widehat{E}_{\ddot{e}}^{<\gamma>} \widehat{E}_{i}^{<\beta>}\left(\sigma_{<\beta>} \sigma_{<\gamma>}\right)\right)_{\underline{\tau}}^{\frac{\delta}{\tau}} \bar{E}_{\ddot{u}}^{<\frac{\tau}{\tau}} k_{\ddot{a} \longleftarrow}+ \\
& \widehat{E}_{i}^{<\gamma>}\left(\widehat{E}_{\ddot{u}}^{<\beta>} \widehat{E}_{\ddot{e}}^{<\alpha>} \widehat{\eta}_{<\beta><\alpha>}\right)\left(\sigma_{<\gamma>}\right)^{\underline{\alpha} \underline{\beta}}\left[\varepsilon^{\dddot{\imath} u ̈} \gamma^{\dddot{e} a ̈}(\bar{q}-s) \bar{\Lambda}_{\underline{\alpha}}+\right. \\
& \left.\left.\left.(-\gamma)^{-1 / 2} \varepsilon^{\dddot{\imath} \ddot{u}} \varepsilon^{\dddot{e} \ddot{a}}(q-\bar{s}) \Lambda_{\underline{\alpha}}\right] k_{\ddot{a} \not \underline{\beta}}\right\}+ \text { h.c. }\right)+ \\
& \frac{1}{2} \triangle\left(\sqrt{-\gamma} \gamma^{\dddot{\imath} u}\right) \widehat{E}_{i}^{<\gamma>} \widehat{E}_{\ddot{u}}^{<\beta>} \widehat{\eta}_{<\gamma><\beta>} P+\frac{1}{2} \sqrt{-\gamma} \gamma^{i \dddot{u}} \widehat{E}_{i}^{<\gamma>} \widehat{E}_{\ddot{u}}^{<\beta>} \widehat{\eta}_{<\gamma><\beta>} \triangle P,
\end{aligned}
$$

where h.c. denotes Hermitian conjugation.

Using relation (67) and fixing the $U(1)$-gauge as to have

$$
P=q-\bar{s}=\bar{q}-s \text { and } \triangle P=(q-\bar{s})\left(\Delta E^{\underline{\alpha}} \Lambda_{\underline{\alpha}}-\Delta \bar{E}^{\underline{\alpha}} \bar{\Lambda}_{\underline{\alpha}}\right)
$$

we can obtain zero values of the coefficients before $\left(\sigma_{a_{p} b_{p}}\right)$-terms. The rest of terms in $\triangle L$ vanish for a corresponding fixing of the variation $\triangle\left(\sqrt{-\gamma} \gamma^{i \ddot{u}}\right)$.

So, in this section we have constructed a model of higher order anisotropic IIB-superstring on the background of IIB supergravity with broken chiral $U(1)-$ subgroup of the supersymmetry $S U(1,1)$-group of automorphisms of $N=$ $2, d=n+m=10$ supergravity. We omit in this works calculus for supersymmetric $\beta$-functions. 


\section{Fermi Strings in Higher Order Anisotropic Spaces}

There are some types of Fermi strings in dependence of the number $\mathrm{N}=0,1,2,4$ of supersymmetry generators (see, for instance, [54, 63, 64 for reviews and basic references on this classification for locally isotropic strings). The aim of this section is to present basic results on Fermi and heterotic strings on higher order anisotropic backgrounds: the construction of actions and calculation of superconformal anomalies.

We note that there are two possible interpretations of models considered in this work. On one hand they can be considered as locally anisotropic supersymmetric two dimensional supersymmetric nonlinear sigma models connected with supergravity. Under quantization of such type theories the superconformal invariance is broken; the two point functions of graviton and gravitino, computed from the quantum effective action can became nontrivial in result (this conclusion was made [1]] for locally isotropic sigma models and, in general, holds good for locally anisotropic generalizations). On the other hand our models can be interpreted as Fermi strings on higher order anisotropic background. We shall follow the second treatment.

The effective "off-shell" action $\Gamma$ for a (infinite) set of locally anisotropic fields is introduced (in a manner similar to [39]) as

$$
\begin{gathered}
\Gamma[G, H, \ldots]=\sum_{\chi} e^{\tilde{\sigma} \chi} \int\left[D \gamma_{\ddot{e} i}^{(e)}\right]\left[D u^{<\alpha>}\right] \exp (-I), \\
I=\frac{1}{2 \pi \alpha^{\prime}} \int d^{2} z\left\{\frac{1}{2} \sqrt{\gamma^{(e)}} \gamma_{(e)}^{\dddot{a} i} \partial_{\ddot{a}} \widehat{u}^{<\alpha>} \partial_{i} \widehat{u}^{<\beta>} G_{<\alpha><\beta>}(u)+\right. \\
\left.\varepsilon^{\dddot{a} i} \partial_{\ddot{a}} \widehat{u}^{<\alpha>} \partial_{i} \widehat{u}^{<\beta>} H_{<\alpha><\beta>}(u)+\ldots\right\},
\end{gathered}
$$

where dots are used instead of possible sources (with higher order derivations), compatible with the reparametrization invariance, of another types of perturbations and $\gamma_{\ddot{e i}}^{(e)}$ is the Euclid two dimensional metric. In (69) we consider in explicit form the components of locally anisotropic graviton and antisymmetric d-tensor and, for simplicity, omit the dilaton field and topological considerations.

The problem of calculation of $\Gamma$ is split into two steps: the first is the calculation of the effective action on an higher order anisotropic with fixed Euler characteristic $\chi$ then the averaging on all metrics and topologies. In order to solve the first task we shall compute

$$
\exp \{-W[G, H, g]\}=\int[D \eta] \exp \{-I[u+v(z), g]\}
$$




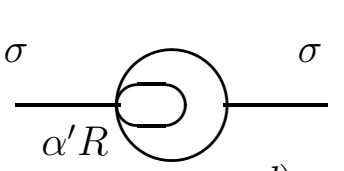

d)

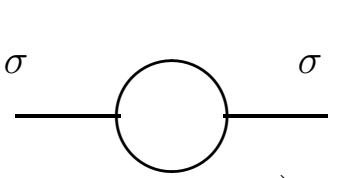

a)

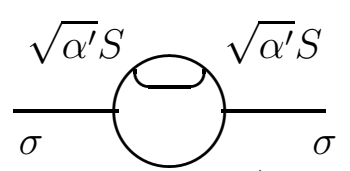

e)

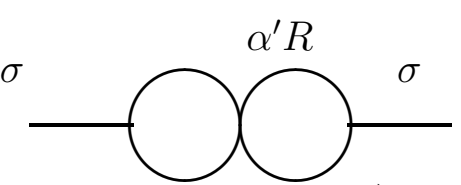

b)
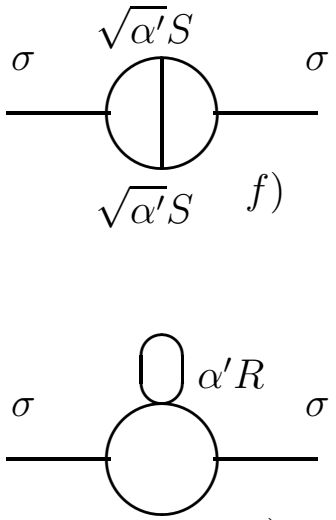

c)

Figure 1: The diagrams defining the conformal anomaly of a closed boson string in a higher order anisotropic space

The general structure $W$ is constructed from dimension and symmetry considerations 85

$$
W=-\frac{\beta(u)}{\varepsilon} \int R(z) \sqrt{\gamma_{(e)}} d^{2} z+\gamma(u) \int\left(R(z) \sqrt{\gamma_{(e)}}\right)_{z} \square_{z z^{\prime}}^{-1}\left(R(z) \sqrt{\gamma_{(e)}}\right)_{z^{\prime}} d^{2} z d^{2} z^{\prime},
$$

where the dimensional regularization $\left(\varepsilon=2-d_{2}\right.$ on world sheet), $\gamma_{(e)}$ is the determinant of the two dimensional metric, $R=R_{a \ddot{a} i}^{a ̈}$, one holds the relation $\beta(u)=4 \gamma(u)$ for dimensionless functions (we can computer them as perturbations on $\alpha^{\prime}$ ) and note that the second term in (73) is the Weyl anomaly which shall be computed by using normal locally adapted to $\mathrm{N}$-connection coordinates on higher order anisotropic space.

For a two-dimensional conformal flat two-dimensional, $d_{2}=2$, metric $\gamma_{\ddot{e} i}^{(e)}$ we find

$$
\gamma_{\ddot{e} \bar{i}}^{(e)}=e^{2 \sigma} \delta_{\ddot{e i}}, R(u)=-2 e^{-2 \sigma} \square \sigma, \gamma_{(e)}^{\dddot{a ̈}}=e^{-2 \sigma} \delta^{\dddot{a ̈}}, \sqrt{\gamma_{(e)}}=e^{2 \sigma} .
$$

From decomposition $\gamma_{\ddot{e} i}^{(e)}=\delta_{\ddot{e} i}+h_{\ddot{e} i}$ with respect to first and second order terms on $h$ we have

$$
\sqrt{\gamma^{(e)}} \gamma_{(e)}^{\ddot{a} i}=\left[1+\varepsilon \sigma(z)+\frac{\varepsilon}{2}(d-4) \sigma^{2}(z)\right] \delta^{\ddot{a} i}
$$

when $h_{\ddot{e} i}=2 \sigma h_{\ddot{e} \ddot{i}}, h \equiv h_{\ddot{a}}^{\ddot{a}}=2 \sigma d_{(2)}$. We give similar formulas for the frame decomposition of two metric

$$
e^{1 / 2} e_{\underline{\ddot{e}}}^{\ddot{e}}=\left(1+\frac{1}{2} \varepsilon \sigma\right) \delta_{\underline{\ddot{e}}}^{\ddot{e}}, e^{-1 / 2} e_{\ddot{e} \underline{e}}=\left(1-\frac{1}{2} \varepsilon \sigma\right) \delta_{\ddot{e} \ddot{e}},
$$


which are necessary for dealing with spinors in curved spaces.

Transforming the quantum field $\zeta \rightarrow \sqrt{2 \pi \alpha^{\prime}} \zeta$, where $\zeta$ is the tangent $\mathrm{d}-$ vector in the point $u \in \mathcal{E}^{<z>}$ of the higher order anisotropic space and using the conformal-flat part of the two dimensional metric we obtain this effective action necessary for further calculations

$$
\begin{gathered}
I_{\text {int }}^{e f f}=\int d^{2} z\left[\frac{1}{3}\left(2 \pi \alpha^{\prime}\right)^{1 / 2} \varepsilon^{\ddot{e} i} H_{<\alpha><\beta><\gamma>}(u) \partial_{\ddot{e}} \zeta^{<\alpha>} \partial_{i} \zeta^{<\beta>} \zeta^{<\gamma>}+\right. \\
\frac{2 \pi \alpha^{\prime}}{6} \varepsilon \sigma(z) R_{<\alpha><\beta><\gamma><\delta>}(u) \partial_{\ddot{e}} \zeta^{<\alpha>} \partial_{\ddot{e}} \zeta^{<\delta>} \zeta^{<\beta>} \zeta^{<\gamma>}+ \\
\frac{1}{2} \varepsilon \sigma(z) \partial_{\ddot{e}} \zeta^{<\alpha>} \partial_{\ddot{e}} \zeta^{<\alpha>}+\frac{2 \pi \alpha^{\prime}}{6} R_{<\alpha><\beta><\gamma><\delta>}(u) \partial_{\ddot{e}} \zeta^{<\alpha>} \partial_{\ddot{e}} \zeta^{<\delta>} \zeta^{<\beta>} \zeta^{<\gamma>}+ \\
\frac{2 \pi \alpha^{\prime}}{4} D_{<\alpha>} H_{<\alpha><\beta><\gamma>}(u) \varepsilon^{\dddot{e} i} \partial_{\ddot{e}} \zeta^{<\beta>} \partial_{\ddot{i}} \zeta^{<\gamma>} \zeta^{<\alpha>} \zeta^{<\delta>},
\end{gathered}
$$

where $(u)$ higher order anisotropic space coordinates not depending on two coordinates $z$. So, the anomaly in (74) takes the form

$$
4 \vartheta \int d^{2} z \sigma(z) \square \sigma(z)
$$

If $\widetilde{\sigma}$ in (70) is the connection on topologies constant the first term in (17) can be absorbed by the renormalization of this connection constant. The set of two-loop diagrams defining (73) is illustrated in the figure 1 . We note that we must take into account tedpoles because of the compactness of the string world sheet there are not infrared divergences.

In the one-loop approximation (figure 1) we find

$$
\beta^{(1)}=\frac{1}{24 \pi}\left(n+m_{1}+\ldots+m_{z}\right), \gamma^{(1)}=\frac{1}{96 \pi}\left(n+m_{1}+\ldots+m_{z}\right) ;
$$

there is correspondence with classical results [85] if we consider a trivial distinguishing of the space-time dimension $n_{E}=n+m_{1}+\ldots+m_{z}$.

The two-loop terms (figure 1) from (73) are computed as

$$
\begin{gathered}
b)=-\frac{\pi \alpha^{\prime} \varepsilon^{2} R}{3(2 \pi)^{6}} \int d^{2} k \sigma(k) \sigma(-k) \int d^{d_{2}} p d^{d_{2}} q \times \\
\frac{\left(p \cdot k-p^{2}\right)^{2}\left(q \cdot k-q^{2}\right)+\left(p \cdot k-p^{2}\right)^{2}(p \cdot q)\left(q \cdot k-q^{2}\right)}{p^{2}(k-p)^{2} q^{2}(k-q)^{2}} \\
c)=\frac{\pi \alpha^{\prime} \varepsilon^{2} R}{3(2 \pi)^{4}} G(0) \int d^{2} k \sigma(k) \sigma(-k) \int d^{d_{2}} p \frac{\left(p \cdot k-p^{2}\right)^{2}}{p^{2}(k-p)^{2}}
\end{gathered}
$$




$$
\begin{aligned}
& d)=-\frac{\pi \alpha^{\prime} \varepsilon^{2} R}{3(2 \pi)^{4}} G(0) \int d^{2} k \sigma(k) \sigma(-k) \int d^{d_{2}} p \frac{\left(p \cdot k-p^{2}\right)^{2}}{p^{2}(k-p)^{2}}, \\
& e)+f)=\frac{2 \varepsilon^{2} \pi \alpha^{\prime} H_{<\alpha><\beta><\gamma>}^{2}}{(2 \pi)^{6}} \varepsilon^{\dddot{e ̈}} \varepsilon^{\ddot{a} \ddot{i}} \int d^{2} k \sigma(k) \sigma(-k) \int d^{d_{2}} p d^{d_{2}} q \times \\
& \frac{1}{p^{2}(k-p)^{2} q^{2}(k-p-q)^{2}} \times \\
& \left\{\frac{\left(k \cdot p-p^{2}\right)\left(k \cdot q-q^{2}\right) p_{\ddot{e}}(k-q)_{i}(k-q)_{\ddot{a}} q_{\ddot{u}}}{(k-q)^{2}}+\right. \\
& \left.\frac{\left(k \cdot p-p^{2}\right)^{2} q_{\ddot{e}}(k-q)_{\ddot{i}} q_{\ddot{a}}(k-p)_{\ddot{u}}}{(k-p)^{2}}\right\}
\end{aligned}
$$

where

$$
\sigma(z) \equiv \frac{1}{(2 \pi)^{2}} \int d^{2} p \sigma(p) \exp (-i p x)
$$

The contributions of tedpole nonvanishing diagrams mutually compensate. The sum of the rest of contributions results in the anomaly

$$
\gamma^{(2)}=\frac{\alpha^{\prime}}{64 \pi}\left(-R+\frac{1}{3} H^{2}\right)=\frac{\alpha^{\prime}}{64 \pi}\left(-\widehat{R}-\frac{2}{3} H^{2}\right),
$$

where $H^{2}=H_{\langle\alpha\rangle<\beta><\gamma>} H^{<\alpha><\beta><\gamma>}$ and $\widehat{R}$ is the scalar curvature with torsion.

Computing $W$ in the leading order on $\alpha^{\prime}$ for the closed boson string, it is not difficult to find the effective action $\Gamma$ for massless perturbations of the string (of the metric $G_{<\alpha><\beta>}$ and field $H_{<\alpha><\beta>}$ ) on the tree $(\chi=2)$ level. Taking into account the identity

$$
\int\left(R(z) \sqrt{\gamma_{(e)}}\right)_{z} \square_{z z^{\prime}}^{-1}\left(R(z) \sqrt{\gamma_{(e)}}\right)_{z^{\prime}} d^{2} z d^{2} z^{\prime}=16 \pi
$$

for the metric on sphere we find

$$
\Gamma^{(0)}[G, H] \sim \int \frac{\delta^{n_{E}} u}{\left(2 \pi \alpha^{\prime}\right)^{n_{E} / 2}} \sqrt{G(u)}\left[1+\frac{\alpha^{\prime}}{4}\left(-R+\frac{1}{3} H^{2}\right)\right],
$$

where $n_{E}=n+m_{1}+\ldots+m_{z}$ is the dimension of higher order anisotropic space. Formula (74) generalizes for such type of spaces (scalar curvature $R$ and torsion $H$ are for, distinguished by $\mathrm{N}$-connection, on la-space) of that presented in [27, 28]. The cosmological constant in (74) arises due to the taxion modes in the spectrum of boson strings and is absent for superstrings. From vanishing of $\beta$-functions for $\widehat{R}$ and tacking into account the contributions of 
reparametrization ghosts 85 into the anomaly of boson string we obtain into the leading approximation

$$
\begin{aligned}
& \beta=\frac{n_{E}-8}{24 \pi}+\frac{\alpha^{\prime}}{16 \pi}\left(-\widehat{R}-\frac{2}{3} H^{2}\right)+\ldots \\
& \gamma=\frac{n_{E}-26}{96 \pi}+\frac{\alpha^{\prime}}{64 \pi}\left(-\widehat{R}-\frac{2}{3} H^{2}\right)+\ldots
\end{aligned}
$$

In consequence, the correction to the critical dimension is

$$
D_{c}=26+\alpha^{\prime} H^{2}+O\left(\left[\alpha^{\prime}\right]^{2}\right) \text {. }
$$

We emphasize that torsion in (75) can be interpreted in a different manner that in the case of locally isotropic theories where $H_{\ldots}$ is considered as an antisymmetric strength of a specific gauge field (see the Wess-Zumino-Witten model [124, 127]). For locally anisotropic spaces we suggested the idea that the $H_{\ldots}$ terms are induced by the distinguished components of torsions of, in our case, higher order anisotropic spaces.

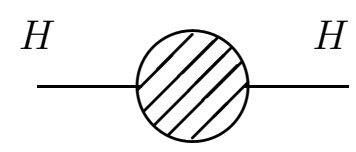

Figure 2: The diagrams defining supergravitational and superconformal anomalies in the theory of higher order anisotropic superstrings

The presented in this section constructions can be generalized for the case of $\mathrm{N}=1$ and $\mathrm{N}=2$ higher order anisotropic Fermi strings. Let decompose action $I_{S}\left[e_{\ddot{e}}^{\ddot{e}}, u+\zeta(z), \psi(z)\right]$ to within forth order on quantum fields $\zeta^{<\alpha>}$ and $\psi_{\ddot{e}}^{<\alpha>}(z)$. After redefinition $\zeta \rightarrow \sqrt{2 \pi \alpha^{\prime}} \zeta, \psi \rightarrow e^{-1 / 4} \sqrt{2 \pi \alpha^{\prime}} \psi$ we obtain the next additional to (72) term:

$$
\begin{gathered}
I_{F(\text { int })}=\int d^{2} z\left[\frac{i}{4} \varepsilon \sigma \bar{\psi}^{<\underline{\alpha}>} \gamma^{\ddot{e}} \partial_{\ddot{e}} \psi^{<\underline{\alpha}>}-\right. \\
\frac{i}{2} \sqrt{2 \pi \alpha^{\prime}}\left(1-\frac{\varepsilon}{2} \sigma\right) H_{<\alpha><\beta><\gamma>} \bar{\psi}^{<\alpha>} \gamma^{\ddot{e}}\left(\partial_{\ddot{e} \zeta} \zeta^{<\gamma>}\right) \psi^{<\beta>}+ \\
i \frac{\pi \alpha^{\prime}}{2}\left(1+\frac{\varepsilon}{2} \sigma\right) R_{<\alpha><\beta><\gamma><\delta>} \bar{\psi}^{<\alpha>} \gamma^{\ddot{e}}\left(\partial_{\ddot{e}} \zeta^{<\delta>}\right) \psi^{<\beta>} \zeta^{<\gamma>}+ \\
\left.\frac{\pi \alpha^{\prime}}{16} \widehat{R}_{<\alpha><\beta><\gamma><\delta>} \bar{\psi}^{<\alpha>}\left(1+\gamma_{5}\right) \psi^{<\gamma>} \bar{\psi}^{<\beta>}\left(1+\gamma_{5}\right) \psi^{<\delta>}\right] .
\end{gathered}
$$


Fixing the gauges

$$
\begin{gathered}
\mathrm{N}=1: e_{\ddot{e}}^{\ddot{e}}=e^{\sigma} \delta_{\ddot{e}}^{\ddot{e}} \psi_{\ddot{e}}=\frac{1}{2} \gamma_{\ddot{e}} \lambda, \\
\mathrm{N}=2: e_{\ddot{e}}^{\ddot{e}}=e^{\sigma} \delta_{\ddot{e}}^{\ddot{e}} \psi_{\ddot{e}}=\frac{1}{2} \gamma_{\ddot{e}} \lambda, A^{\ddot{e}}=\frac{1}{2} \varepsilon^{\ddot{e} \ddot{u}} \partial_{\ddot{u}} \rho,
\end{gathered}
$$

where $\lambda$ is the Maiorana $(N=1)$ or $\operatorname{Dirac}(N=2)$ spinor. Because of supersymmetry it is enough [39] to computer only the coefficient before Weyl anomaly in order to get the superconformal anomalies. The one-loop results are

$$
\begin{aligned}
& \mathrm{N}=1: \beta^{(1)}=\frac{n_{E}}{16 \pi}, \gamma^{(1)}=\frac{n_{E}}{64 \pi}, \\
& \mathrm{N}=2: \beta^{(1)}=\frac{n_{E}}{8 \pi}, \gamma^{(1)}=\frac{n_{E}}{32 \pi},
\end{aligned}
$$

from which, taking into account the reparametrization and superconformal ghosts we obtain these values of critical dimension $(\gamma=0)$ :

$$
\begin{gathered}
\mathrm{N}=1: \beta_{t}^{(1)}=\frac{n_{E}-2}{16 \pi}, \gamma^{(1)}=\frac{n_{E}-10}{64 \pi}, \\
\mathrm{N}=2: \beta^{(1)}=\frac{n_{E}}{8 \pi}, \gamma^{(1)}=\frac{n_{E}-2}{32 \pi} .
\end{gathered}
$$

From formal point of view in the two-loop approximation we must consider diagrams b)-m) from fig. 2. By straightforward calculations by using methods similar to those presented in [39 we conclude that all two-loop contributions b) $-\mathrm{m}$ ) vanish. In result we conclude that for Fermi strings one holds the next formulas:

or

$$
\begin{gathered}
\mathrm{N}=1: \beta=\frac{n_{E}-2}{16 \pi}-\frac{\alpha^{\prime} H^{2}}{24 \pi}+\ldots, \gamma=\frac{n_{E}-10}{64 \pi}-\frac{\alpha^{\prime} H^{2}}{96 \pi}, \\
\mathrm{N}=2: \beta=\frac{n_{E}}{8 \pi}-\frac{\alpha^{\prime} H^{2}}{24 \pi}+\ldots, \gamma=\frac{n_{E}-2}{32 \pi}-\frac{\alpha^{\prime} H^{2}}{96 \pi}
\end{gathered}
$$

$$
\begin{gathered}
\mathrm{N}=1: D_{c}=10+\frac{2}{3} \alpha^{\prime} H^{2}+\ldots, \\
\mathrm{N}=2: D_{c}=2+\frac{1}{3} \alpha^{\prime} H^{2} \ldots
\end{gathered}
$$

in the leading order on $\alpha^{\prime}$.

Finally, we note that because $H^{2}$ contains components of $\mathrm{N}$-connection and torsion of $\mathrm{d}$-connection on higher order anisotropic space we conclude that a possible local anisotropy of space-time can change the critical dimension of Fermi strings. 


\section{Anomalies in Locally Anisotropic $\sigma$-Models}

Anomalies in quantum field theories are considered beginning with works [2] and [17]. Conformal and gravitational anomalies have been analyzed in [32, 37, 3] (see also reviews [129, 80, 63, 64, 44]). The aim of this section is to investigate anomalies in higher order anisotropic $(1,0)$-superspaces.

\subsection{One-loop calculus}

Supergravitational and conform anomalies of heterotic locally anisotropic $\sigma-$ models connected with $(1,0)$ higher order anisotropic supergravity are defined by the finite (anomaly) parts of diagrams of self-energy type (fig. 2). In order to compute anomalies it is necessary to consider all the vertexes of the theory with no more than the linear dependence on potentials $H_{+}^{=}, H_{=}^{\ddagger}$ (see subsection 6.2 and section 9 for denotations on higher order anisotropic heterotic superstrings). We consider this "effective" action (without ghosts):

$$
I=I_{0}+I_{i n t}, I_{i n t}=I_{0}^{\prime}+I_{1}
$$

where

$$
\begin{aligned}
& I_{0}=-\int d^{3} z^{-}\left[i D_{+} \zeta^{<\alpha>} \partial_{=} \zeta^{<\beta>} \widehat{g}_{<\alpha><\beta>}+\chi_{-}^{|I|} D_{+} \chi_{-}^{|I|}\right] \text {, } \\
& I_{0}^{\prime}=-\frac{1}{2} \int d^{3} z^{-}\left\{i D _ { + } \zeta ^ { < \alpha > } \partial _ { = } \zeta ^ { < \beta > } \left(\zeta^{<\gamma>} \widehat{A}_{<\gamma><\alpha><\beta>}+\right.\right. \\
& \zeta^{<\delta>} \zeta^{<\gamma>} \widehat{B}_{\langle\delta><\gamma><\alpha><\beta>}+\zeta^{<\varepsilon>} \zeta^{<\delta>} \zeta^{<\gamma>} \widehat{\mathcal{D}}_{\langle\varepsilon><\delta><\gamma><\alpha><\beta>}+ \\
& \left.\zeta^{<\tau>} \zeta^{<\varepsilon>} \zeta^{<\delta>} \zeta^{<\gamma>} \widehat{M}_{<\varepsilon><\delta><\gamma><\tau><\alpha><\beta>}\right)+\chi_{(-)}^{|I|} \chi_{(-)}^{|J|} D_{+} \zeta^{<\beta>} \times \\
& \left(\zeta^{<\alpha>} \widehat{C}_{<\alpha><\beta>}^{|I||J|}+\zeta^{<\gamma>} \zeta^{<\alpha>} \widehat{E}_{<\gamma><\alpha><\beta>}^{|I||J|}+\zeta^{<\varepsilon>} \zeta^{<\gamma>} \zeta^{<\alpha>} \widehat{K}_{<\varepsilon><\gamma><\alpha><\beta>}^{|I||J|}\right), \\
& I_{1}=-\frac{1}{2} \int d^{3} z^{-}\left\{\left(\widehat{g}_{<\alpha><\beta>}+\zeta^{<\gamma>} \widehat{A}_{<\gamma><\alpha><\beta>}+\right.\right. \\
& \zeta^{<\delta>} \zeta^{<\gamma>} \widehat{B}_{<\delta><\gamma><\alpha><\beta>}+\zeta^{<\varepsilon>} \zeta^{<\delta>} \zeta^{<\gamma>} \widehat{\mathcal{D}}_{<\varepsilon><\delta><\gamma><\alpha><\beta>}+ \\
& \left.\zeta^{<\tau>} \zeta^{<\varepsilon>} \zeta^{<\delta>} \zeta^{<\gamma>} \widehat{M}_{\langle\varepsilon><\delta><\gamma><\tau><\alpha><\beta>}\right)+\left[i H_{+}^{=} \partial_{=} \zeta^{<\alpha>} \partial_{=} \zeta^{<\beta>}+\right. \\
& \left.\frac{1}{2} D_{+} \zeta^{<\alpha>}\left(D_{+} H_{=}^{\ddagger}\right) D_{+} \zeta^{<\beta>}+i\left(D_{+} \zeta^{<\alpha>}\right) H_{=}^{\ddagger}\left(\partial_{\ddagger} \zeta^{<\beta>}\right)\right]+ \\
& \chi_{(-)}^{|I|} H_{+}^{=} \partial_{=} \chi_{(-)}^{|J|}+\chi_{(-)}^{|I|} \chi_{(-)}^{|J|} H_{+}^{=} \partial_{=} \zeta^{<\beta>} \times \\
& \left(\zeta^{<\alpha>} \widehat{C}_{<\alpha><\beta>}^{|I||J|}+\zeta^{<\gamma>} \zeta^{<\alpha>} \widehat{E}_{<\gamma><\alpha><\beta>}^{|I||J|}+\zeta^{<\varepsilon>} \zeta^{<\gamma>} \zeta^{<\alpha>} \widehat{K}_{<\varepsilon><\gamma><\alpha><\beta>}^{|I||J|}\right)+ \\
& \left.\frac{i}{4 \pi} \sum_{p}^{6} \frac{1}{p !} \zeta^{<\alpha_{1}>} \ldots \zeta^{<\alpha_{p}>} \widehat{\mathcal{D}}_{<\alpha_{1}>} \ldots \widehat{\mathcal{D}}_{<\alpha_{p}>} \Phi\left(D_{+} \partial_{\ddagger} H_{=}^{\ddagger}+\partial_{=}^{2} H_{+}^{=}\right)\right\},
\end{aligned}
$$


where

$$
\begin{aligned}
& \widehat{A}_{\langle\gamma\rangle\langle\alpha><\beta\rangle}=\frac{2}{3} \widehat{H}_{\langle\gamma\rangle\langle\alpha\rangle<\beta>,} \\
& \widehat{B}_{\langle\delta\rangle\langle\gamma\rangle\langle\alpha\rangle\langle\beta\rangle}=\frac{1}{3} \widehat{R}_{\langle\delta\rangle\langle\alpha\rangle\langle\beta\rangle\langle\gamma\rangle}+\frac{1}{2} \widehat{\mathcal{D}}_{\langle\delta\rangle} \widehat{H}_{\langle\gamma\rangle\langle\alpha\rangle\langle\beta\rangle,} \\
& \widehat{\mathcal{D}}_{\langle\varepsilon\rangle<\delta><\gamma><\alpha><\beta>}=\frac{1}{5} \widehat{\mathcal{D}}_{<\varepsilon\rangle} \widehat{\mathcal{D}}_{<\delta>} \widehat{H}_{\langle\gamma\rangle<\alpha><\beta>}+
\end{aligned}
$$

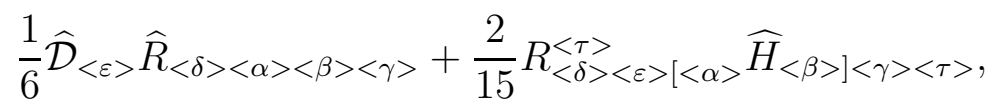

$$
\begin{aligned}
& \widehat{M}_{\langle\varepsilon><\delta>\langle\gamma\rangle\langle\tau><\alpha><\beta>}=\frac{1}{20} \widehat{\mathcal{D}}_{\langle\varepsilon\rangle} \widehat{\mathcal{D}}_{<\delta>} \widehat{R}_{<\gamma><\alpha><\beta><\tau>}+ \\
& \frac{2}{45} \widehat{R}_{\langle\beta\rangle\langle\tau\rangle\langle\gamma\rangle\langle\vartheta\rangle} R_{<\delta>\langle\varepsilon\rangle\langle\alpha\rangle}^{<\vartheta>}+\widehat{\mathcal{D}}_{\langle\varepsilon\rangle} \widehat{\mathcal{D}}_{<\delta>} \widehat{\mathcal{D}}_{\langle\gamma\rangle} \widehat{H}_{\langle\tau\rangle\langle\alpha\rangle\langle\beta\rangle}+
\end{aligned}
$$

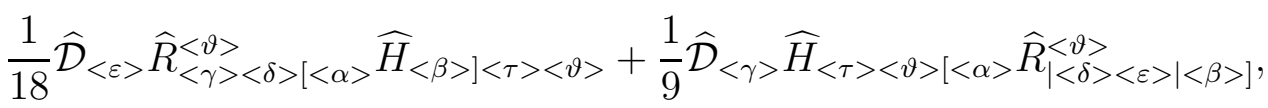

$$
\begin{aligned}
& \widehat{C}_{\langle\alpha\rangle\langle\beta\rangle}^{|I||J|}=-\frac{1}{2} \widehat{F}_{\langle\alpha\rangle\langle\beta\rangle}^{|I||J|}, \widehat{E}_{\langle\gamma\rangle\langle\alpha\rangle\langle\beta\rangle}^{|I||J|}=-\frac{1}{3} \widehat{\mathcal{D}}_{\langle\gamma\rangle} \widehat{F}_{\langle\alpha\rangle\langle\beta\rangle}^{|I||J|}, \\
& \widehat{K}_{\langle\varepsilon\rangle\langle\gamma\rangle\langle\alpha\rangle\langle\beta\rangle}^{|I||J|}=-\frac{1}{24}\left(3 \widehat{\mathcal{D}}_{\langle\varepsilon\rangle} \widehat{\mathcal{D}}_{\langle\gamma\rangle} \widehat{F}_{\langle\alpha\rangle\langle\beta\rangle}^{|I||J|}+\widehat{F}_{\langle\varepsilon>\langle\tau\rangle}^{|I||J|} R_{\langle\alpha\rangle\langle\gamma\rangle\langle\beta\rangle}^{<\tau>} .\right.
\end{aligned}
$$

For simplicity, in this section we shall omit tilde "»" over geometric objects (such as curvatures and torsions computed for Christoffel distinguished symbols (39)) but maintain hats "\% in order to point out even components on the s-space).

We write the supergravitational anomaly in this general form (see [124 for locally isotropic models):

$$
\frac{1}{32 \pi} \int d^{3} z^{-}\left\{\gamma_{1} D_{+} H_{+}^{=} \frac{\partial_{=}^{4}}{\square} H_{+}^{=}-i \gamma_{2} D_{+} H_{=}^{\ddagger} \frac{\partial_{=}^{3}}{\square} H_{=}^{\ddagger}\right\},
$$

where background depending coefficients will be defined from a perturbation calculus on $\alpha^{\prime}$ by using (76).

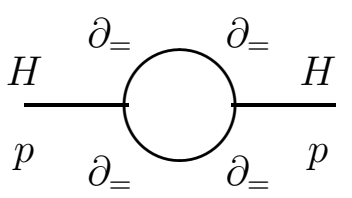

Figure 3: $(1,0)$ supergraf defining the one-loop anomaly

For computation of supergrafs we use a standard techniques [40] of reducing 

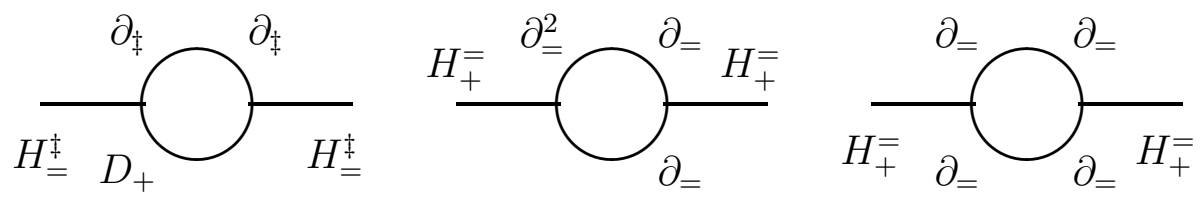

Figure 4: (1,0) supergrafs defining the one-loop dilaton contribution to the anomaly

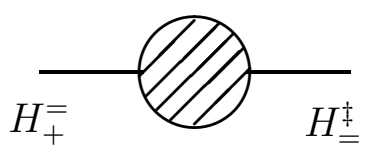

Figure 5: The diagrams being unessential for calculation of anomalies

to integrals in momentum space which is standard practice in quantum field theory. For instance, the one loop diagram corresponding to figure 3 is computed

$$
\frac{1}{(2 \pi)^{2}} \int d^{2} k \frac{k_{=}^{2}\left(k_{=}+p_{=}\right)^{2}}{k^{2}(k+p)^{2}}=-\frac{i}{24 \pi} \frac{p_{=}^{4}}{p^{2}} .
$$

Diagrams of type illustrated on fig. 4 give rise only to local contributions in the anomaly and are not considered because of dimensional considerations.

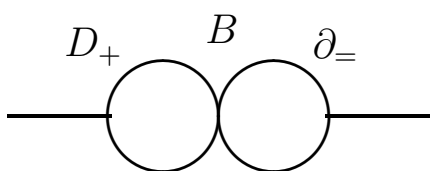

a)

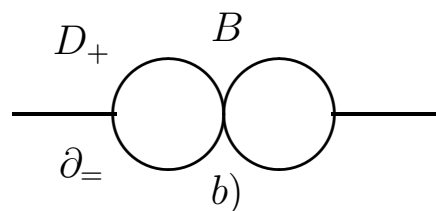

b)

Figure 6: B-depending two-loop corrections to the anomaly

We note that comparing with similar locally isotropic results 64 the torsions $H$ : are generated by components of the distinguished torsion of the higher order anisotropic background.

\subsection{Two-loop calculus}

The two-loop $B$-depending corrections to the anomaly are defined by diagrams illustrated in fig. 6. The one-loop results including ghosts (56) are similar to 
locally isotropic ones [40]. Thus we present a brief summary (we must take into account the splitting of dimensions in higher order anisotropic spaces):

$$
\begin{gathered}
W_{\text {eff }}^{1-\text { loop }}=\frac{1}{96 \pi} \int d^{3} z^{-}\left\{\left(n+m_{1}+\ldots+m_{z}-26+\frac{N_{E}}{2}\right) D_{+} H_{+}^{=} \frac{\partial_{=}^{4}}{\square} H_{+}^{=}-\right. \\
\left.\frac{3 i}{2}\left(n+m_{1}+\ldots+m_{z}-10\right) D_{+} H_{=}^{\ddagger} \frac{\partial_{\ddagger}^{3}}{\square} H_{=}^{\ddagger}\right\} .
\end{gathered}
$$

If the action (77) is completed by local conterterms

$$
H_{+}^{=} \square H_{=}^{\ddagger}, S \partial_{=}^{2} H_{+}^{=}, S \partial_{\ddagger} D_{+} H_{=}^{\ddagger}, S \partial_{=} D_{+} S
$$

and conditions $N_{E}-\left(n+m_{1}+\ldots+m_{z}\right)=22$ and $\gamma_{1}=\gamma_{2} \equiv \gamma$ are satisfied, we obtain from (77) a gauge invariant action:

$$
W_{e f f}+W_{l o c}=\frac{1}{16 \pi}\left(n+m_{1}+\ldots+m_{z}-10\right) \int d^{3} z^{-} \Sigma^{+}\left(\frac{D_{+}}{\square}\right) \Sigma^{+}
$$

The action (78) is the conformal anomaly of our model $\left(\Sigma^{+}\right.$depends on $\left.S\right)$. The critical parameters of the higher order anisotropic heterotic string

$$
n+m_{1}+\ldots+m_{z}=10 \text { and } N_{E}=32
$$

make up the conditions of cancelation of it anomalies (the original locally isotropic result was obtained in [46]). We can also compute and add the oneloop dilaton (see fig. 5) contribution to (78):

$$
\begin{gathered}
W_{\text {eff }}^{(1, \Phi)}=\frac{\mathcal{D}^{2} \Phi}{128 \pi^{2}} \int d^{3} z^{-}\left[i D_{+} H_{=}^{\ddagger} \frac{\partial_{\ddagger}^{3}}{\square} H_{=}^{\ddagger}-D_{+} H_{+}^{=} \frac{\partial_{=}^{4}}{\square} H_{+}^{=}\right], \\
\gamma^{(1, \Phi)}=-\frac{1}{4 \pi} \mathcal{D}^{2} \Phi .
\end{gathered}
$$

computed

$$
\begin{gathered}
a)=I_{1}(p)=\int \frac{d^{2} k d^{2} q}{16 \pi^{4}} \frac{q_{=}^{2}\left(q_{=}+p_{=}\right)\left(k_{=}+p_{=}\right)}{\left(q^{2}-\mu^{2}\right)\left[(q+p)^{2}-\mu^{2}\right]} \times \\
\frac{k^{2}}{\left(k^{2}-\mu^{2}\right)\left[(k+p)^{2}-\mu^{2}\right]}=\frac{1}{64 \pi^{2}} \frac{p_{=}^{4}}{p^{2}}+O\left(\mu^{2}\right), \\
b)=I_{2}(p)=\int \frac{d^{2} k d^{2} q}{16 \pi^{4}} \frac{q=\left(q=+p_{=}\right)}{\left(q^{2}-\mu^{2}\right)\left[(q+p)^{2}-\mu^{2}\right]} \times \\
\frac{k^{2}\left(k_{=}+p_{=}\right)^{2}}{\left(k^{2}-\mu^{2}\right)\left[(k+p)^{2}-\mu^{2}\right]}=-\frac{1}{32 \pi^{2}} \frac{p_{=}^{4}}{p^{2}}+O\left(\mu^{2}\right),
\end{gathered}
$$



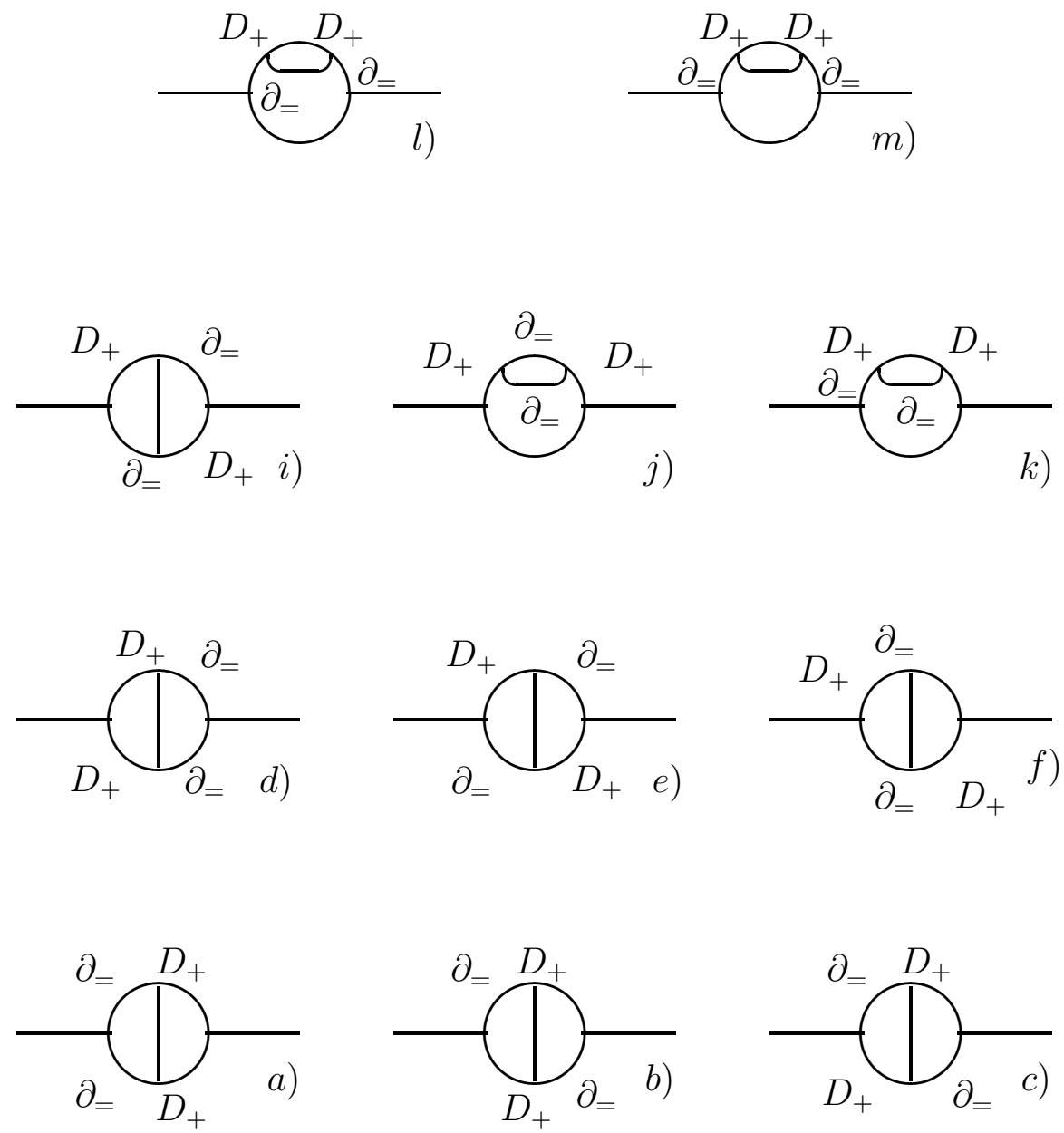

Figure 7: $A^{2}$-depending two-loop corrections to the anomaly 
where the mass parameter $\mu^{2}$ is used as a infrared regulator.

Two-loop $\mathrm{A}^{2}$-dependent diagrams are illustrated in fig. 7. The supergrafs f),i) and j) are given by the momentum integral $I_{f}=I_{i}=I_{j}=I(p)$ :

$$
I(p)=\int \frac{d^{2} k d^{2} q}{(2 \pi)^{4}} \frac{k_{=} q_{=}\left(k_{=}+q_{=}+p_{=}\right)^{2}}{k^{2} q^{2}(k+q+p)^{2}}=\frac{p_{=}^{4}}{96 \pi^{2} p^{2}} ;
$$

to this integral there are also proportional the anomaly parts of diagrams a)e),k),l) and $\mathrm{m}$ ). The rest of possible $A^{2}$-type diagrams do not contribute to the anomaly part of the effective action. After a straightforward computation of two loop diagrams we have

$$
\gamma^{2-l o o p}=\frac{1}{16 \pi}\left(-\widetilde{R}+\frac{1}{3} H^{2}\right) ;
$$

there are not dilaton contributions in the two-loop approximation.

The anomaly coefficient $\gamma$ is connected with the central charge of Virasoro superalgebra of heterotic string on the background of massless modes (under the conditions of vanishing of $\beta$-functions or, equivalently, if the motion equations are satisfied, see [99, 100, 97, 84, 35, 81, 25]):

$$
\begin{gathered}
2 \gamma+\alpha^{\prime}\left(\mathcal{D}_{<\beta>} \Phi\right)^{2}=\widetilde{\beta}^{\Phi} \equiv \beta^{\Phi}-\frac{1}{4} \beta_{<\alpha><\beta>}^{g} g^{<\alpha><\beta>}= \\
\frac{n+m_{1}+\ldots+m_{z}-10}{2}+\frac{\alpha^{\prime}}{2} L_{e f f}, \\
<T>_{-}=\frac{1}{4 \pi} \widetilde{\beta}^{\Phi} \Sigma^{+}+\ldots,
\end{gathered}
$$

where $\left\langle T>_{-}\right.$is the averaged supertrace, $\beta_{<\alpha><\beta>}^{g}$ is the metric $\beta$-function, $\widetilde{\beta}^{\Phi}$ is the dilaton $\beta$-function and by dots there are denoted the terms vanishing on the motion equations. We note that the $\beta$-functions and effective Lagrangian are defined in the string theory only with the exactness of redefinition of fields [25]. From a standard calculus according the perturbation theory on $\alpha^{\prime}$ we have

$$
\beta_{<\alpha><\beta>}^{g}=\alpha^{\prime}\left(\widetilde{R}_{<\alpha><\beta>}-H_{<\alpha><\beta>}^{2}\right)
$$

and

where

$$
\begin{gathered}
S_{\text {eff }}^{(0)} \equiv \int d^{10} X \sqrt{|g|} L_{e f f}^{(0)}= \\
\frac{1}{2} \int d^{10} X \sqrt{|g|}\left\{-\widetilde{R}-4 \mathcal{D}^{2} \Phi+4\left(\mathcal{D}_{<\alpha>} \Phi\right)^{2}+\frac{1}{3} H^{2}\right\}
\end{gathered}
$$

$$
H_{\langle\alpha\rangle\langle\beta\rangle}^{2} \equiv H_{\langle\alpha\rangle\langle\gamma\rangle\langle\delta>} H_{<\beta>}<\gamma><\delta>,
$$

which is a higher order anisotropic generalization of models developed in 20 , 31, 18]. 


\section{Discussion and Conclusions}

We have explicitly constructed a new class of superspaces with higher order anisotropy. The status of the results in this work and the relevant open questions are discussed as follows.

From the generally mathematical point of view it is possible a definition of a supersymmetric differential geometric structure imbedding both type of supersymmetric extensions of Finsler and Lagrange geometry as well various Kaluza-Klein superspaces. The first type of superspaces, considered as locally anisotropic, are characterized by nontrivial nonlinear connection structures and corresponding distinguishing of geometric objects and basic structure equations. The second type as a rule is associated to trivial nonlinear connections and higher order dimensions. A substantial interest for further considerations presents the investigations of physical consequences of models of field interactions on higher and/or lower dimensional superspaces provided with $\mathrm{N}$-connection structure.

It worth noticing that higher order derivative theories are one of currently central division in modern theoretical and mathematical physics. It is necessary a rigorous formulation of the geometric background for developing of higher order analytic mechanics and corresponding extensions to classical quantum field theories. Our results not only contain a supersymmetric extension of higher order fiber bundle geometry, but also propose a general approach to the "physics" with local anisotropic interactions. The elaborated in this work formalism of distinguished vector superbundles highlights a scheme by which supergravitational and superstring theories with higher order anisotropy can be constructed.

To develop in a straightforward manner self-consistent physical theories, define local conservation laws,give a corresponding treatment of geometrical objects and so on, on different extensions on Finsler spaces with nonlinear structure of metric form and of connections, torsions and curvatures is a highly conjectural task. Only the approach on modeling of geometric models of the mentioned type (super)spaces on vector (super)bundles provided with compatible nonlinear and distinguished connections and metric structures make "visible" the possibility (see, for instance, [75, 76, 102, 113, 103, 104, 105, 109, 112]), manner of elaboration, as well common features and differences of models of fundamental physical fields with generic locally anisotropic interactions. From viewpoint of the string theory fundamental ideas only some primarily changes in established material have been introduced in this work. But we did not try to a simple straightforward repetition of standard material in context of some sophisticate geometries. Our main purposes were to illustrate that the higher order anisotropic supergravity is also naturally contained in the framework of low energy superstring dynamics and to develop a corresponding geometric and 
computational technique for supersymmetric sigma models in locally anisotropic backgrounds.

The above elaborated methods of perturbative calculus of anomalies of hetrotic sigma models in higher order anisotropic superspaces, as a matter of principle, can be used in every finite order on $\alpha^{\prime}$ (for instance, by using the decomposition (76) for effective action we can, in a similar manner as for oneand two-loop calculations presented in section 10, find corrections of anomalies up to fifth order inclusive) and are compatible with the well known results for locally isotropic strings and sigma models. We omit such considerations in this work.

\section{References}

[1] E. Abdala and R. S. Jasinschi, Nucl. Phys. B232 (1984) 426

[2] S. Adler, Phys. Rev. 177 (1969) 2426

[3] L. Alvarez-Gaume and E. Witten, Nucl. Phys. Nucl. Phys. 234 (1983) 269

[4] S. P. de Alwis, Phys. Rev. D34 (1986) 3760

[5] P. L. Antonelli and R. Miron (eds), Lagrange and Finsler Geometry, Applications to Physics and Biology (Kluwer Academic Publishers, Dordrecht, Boston, London, 1996)

[6] P. L. Antonelli and T. J. Zastavniak (guest eds), Lagrange Geometry, Finsler Spaces and Noise Applied in Biology and Physics., in Mathematical and Computer Modelling, ed. Rolin, E. Y. 20. N415 (Plenum Press, 1994)

[7] G. S. Asanov, Finsler Geometry, Relativity and Gauge Theories (Reidel, Boston, 1985)

[8] G. S. Asanov and S. F. Ponomarenko, Finsler Bundle on Space-Time. Associated Gauge Fields and Connections (Ştiinţa, Chişinău, 1988) [in Russian]

[9] G. S. Asanov, Fibered Generalization of the Gauge Field Theory. Finslerian and Jet Gauge Fields (Moscow University Press, Moscow, 1989) [in Russian]

[10] J. Atick, A. Dhar and B. Ratra, Phys. Rev. D33 (1986) 2824 
[11] J. Bagger, in Supersymmetry: Proc. NATO Adv. Study Inst., Bonn, Aug. 20-31, 1984 (New York, London, 1985) 45

[12] W. Barthel, J. Reine Angew. Math. 212 (1963) 120

[13] C. Bartocci, U. Bruzzo and D. Hermándes-Ruipérez, The Geometry of Supermanifolds (Kluwer Academic Publishers, Dordrecht, Boston, London, 1991)

[14] A. Bejancu, Finsler Geometry and Applications (Ellis Horwood, Chichester, England, 1990)

[15] A. Bejancu, A New Viewpoint on Differential Geometry of Supermanifolds, I (Timişoara University Press, Romania, 1990)

[16] A. Bejancu, A New Viewpoint on Differential Geometry of Supermanifolds, II (Timişoara University Press, Romania, 1991)

[17] J. S. Bell and R. A. Jackiv, Nuovo Cim. 6047

[18] S. Bellucci, Progr. Theor. Phys. 79 (1988) 1288

[19] F. A. Berezin and D. A. Leites, Doklady Academii Nauk SSSR 224 (1975) 505 [in Russian]; Sov. Math. Dokl. 16 (1975) 1218

[20] E. Bergshoeff, M. de Roo, B. de Wit and P. Nieuwenhuizen, Nucl. Phys. B195 (1982) 97

[21] L. Berwald, Math. Z. 25 (1926) 40; Correction, Math. Z. 26 (1927) 176

[22] G. Yu. Bogoslovsky, Theory of Locally-Anisotropic Space-Time (Izdatel'stvo Moscovskogo Universiteta, Moscow, 1992) [in Russian]

[23] H. W. Braden, Ann. Phys. 171 (1986) 433

[24] R. Brooks, F. Muhammad and S. J. Gates, Nucl. Phys B268 (1986) 599

[25] R. Brustein, D. Nemershansky and S. Yankielowicz, Nucl. Phys. B301 (1988) 224

[26] U. Bruzzo and R. Cianci, Class. Quant. Grav. 1 (1984) 213

[27] C. G. Callan, D. Friedan, E. J. Martinec and M. J. Perry, Nucl. Phys. B262 (1985) 593

[28] C. G. Callan, I. R. Klebanov, and M. J. Perry, Nucl. Phys. B278 (1986) 78 
[29] E. Cartan, Les Espaces de Finsler (Hermann, Paris, 1935)

[30] E. Cartan, Exposés de Géométrie in Series Actualités Scientifiques et Industrielles 79 (1936); reprinted (Herman, Paris, 1971)

[31] G. F. Chapline and N. S. Manton, Phys. Lett. 120 (1983) 105

[32] S. M. Christensen and M. J. Duff, Phys. Lett. B76 (1978) 571

[33] R. Cianci, Introduction to Supermanifolds (Napoli, Bibliopolis, 1990)

[34] M. Crampin, W. Sarlet, and F. Cantrijin, Math. Proc. Camb. Phil. Soc. 99 (1986) 565

[35] G. Curci and G. Paffuti, Nucl. Phys. B286 (1988) 399

[36] T. L. Curtright and C. K. Zachos, Phys. Rev. Lett. 53 (1984) 1799

[37] M. J. Duff, Nucl. Phys. B215 (1977) 334

[38] P. Finsler, Über Kurven und Flächen in Allgemeiner Rämen (Dissertation, Göttingen, 1918); reprinted (Birkhäuser, Basel, 1951).

[39] S. Fradkin and A. A. Tseytlin, Phys. Lett. B158 (1985) 316; Nucl. Phys. B261 (1985) 1

[40] S. J. Gates, M. T. Grisaru, L. Mezincescu and P. K. Townsed, Nucl. Phys., B286 (1986) 1

[41] S. J. Gates, M. T. Grisaru, M. Rocek and W. Siegel, Superspace or One Thousand and One Lessons in Supersymmetry (Cummings Publ. Comp. Inc., Benjamin, 1983)

[42] S. J. Gates, C. M. Hull and M. Rocek, Nucl. Phys., B248 (1984) 157

[43] M. B. Green and J. H. Schwarz, Nucl. Phys. B243 (1984) 285

[44] M. B. Green, J. H. Schwarz and E. Witten, Superstring Theory, vol. 1 and 2 (Cambridge University Press, Cambridge, 1987)

[45] M. T. Grisaru, P. Howe, L. Mezinchescu e.a., Phys. Lett. B162 (1985) 116

[46] D. J. Gross, J. A. Harvey, E. Martinec and R. Rohm, Phys. Rev. Lett. 54 (1985) 502

[47] J. C. Henty, C. M. Hull and P. K. Townsed, Phys. Lett. B185 (1987) 73 
[48] E. d'Hoker and D. H. Phong, Rev. Mod. Phys. 60 (1988) 917

[49] P. S. Howe, Phys. Lett. B70 (1977) 453

[50] P. S. Howe, J. Phys. A12 (1979) 393

[51] P. S. Howe, G. Sierra, Phys. Lett. 148 (1984) 451

[52] P. Howe and P. West, Nucl. Phys. B238 (1984) 181

[53] J. Hoyos, M. Quiros, J. Ramirez Mittelbrunn and F. J. De Uries, J. Math. Phys. 25 (1984) 833; 841; 847

[54] C. M. Hull, Lectures on Non-linear Sigma Models and Strings (DAMPT, Cambridge, England, 1987)

[55] C. M. Hull and P. K. Townsed, Nucl. Phys. B301 (1988) 197

[56] R. S. Ingarden, Tensor N.S. 30 (1976) 201

[57] H. Ishikawa, J. Math. Phys. 22 (1981) 995

[58] A. Jadczic and K. Pilch, Commun. Math. Phys. 78 (1981) 373

[59] V. Kac, Commun. Math. Phys. 53 (1977) 31

[60] A. Kawaguchi, Tensor N. S. 6 (1956) 596

[61] J. Kern, Arch. Math. 25 (1974) 438

[62] S. V. Ketov, Nucl. Phys. 294 (1987) 813

[63] S. V. Ketov, Introduction into Quantum Theory of Strings and Superstrings (Nauka, Novosibirsk, 1990) [in Russian]

[64] S. V. Ketov, Nonlinear Sigma-Models in Quantum Field Theory and String Theory (Nauka, Novosibirsk, 1992) [in Russian]

[65] B. Konstant, in Differential Geometric Methods in Mathematical Physics, Lecture Notes in Mathematics 570 (1977) 177

[66] D. A. Leites, Usp. Math. Nauk 35 (1980) 3 [in Russian]

[67] D. A. Leites, The Theory of Supermanifolds (Petrozavodsk, URSS, 1980) [in Russian]

[68] S. Leng, Differential Manifolds (Reading, Mass, Addison-Wesley, 1972) 
[69] M. de Leon and P. Rodrigues, Generalized Classical Mechanics and Field Theory (North-Holland, 1985)

[70] P. Libermann and Ch. M. Marle, Sympletic Geometry and Analytical Mechanics (D. Reidel Publ. Comp., 1987)

[71] C. Lovelace, Phys. Lett. B35 (1984) 75

[72] Yu. I. Manin, Gauge Fields and Complex Geometry (Nauka, Moscow, 1984) [in Russian]

[73] E. Martinec, Phys. Rev. D28 (1983) 2604

[74] M. Matsumoto, Foundations of Finsler Geometry and Special Finsler Spaces (Kaisisha, Shigaken, 1986)

[75] R. Miron and M. Anastasiei, Vector Bundles. Lagrange Spaces. Application in Relativity (Academiei, Romania, 1987) [in Romanian]

[76] R. Miron and M. Anastasiei, The Geometry of Lagrange Spaces: Theory and Applications (Kluwer Academic Publishers, Dordrecht, Boston, London, 1994)

[77] R. Miron and Gh. Atanasiu, Compendium sur les Espaces Lagrange D'ordre Supérieur, Seminarul de Mecanică. Universitatea din Timişoara. Facultatea de Matematică, 199440 p.

[78] R. Miron and T. Kavaguchi, Int. J. Theor. Phys. 30 (1991) 1521

[79] A. Morimoto, Prolongations of Geometric Structures, (Nagoya Univ., 1969)

[80] A. Yu. Morozov, Uspehy Fizicheskih Nauk, 150 (1986) 337

[81] M. Niedermaier, Phys. Lett. B207 (1988) 145

[82] B. E. W. Nilsson, Nucl. Phys. B188 (1981) 176

[83] D. Olivier, Phys. Rev. D33 (1986) 2462

[84] H. Osborn, Nucl. Phys. 308 (1988) 629

[85] A. M. Polyakov, Phys. Lett. B103 (1981) 207; 211; Gauge Fields and Strings, Contemporary Concepts in Physics (Harwood Acad. Publ., Chur e. a., Switzerland, 1987) 
[86] V. N. Ponomarev, A. O. Barvinsky and Yu. N. Obukhov, Geometrodynamical Methods and Gauge Approach to Gravity Theory (Energoatomizdat, Moscow, 1985) [in Russian]

[87] T. D. Robb and J. G. Taylor, Phys. Lett. B176 (1986) 355

[88] A. Rogers, J. Math. Phys. 21 (1980) 1352

[89] A. Rogers, J. Math. Phys. 22 (1981) 939

[90] H. Rund, The Differential Geometry of Finsler Spaces (Springer-Verlag, Berlin, 1959)

[91] M. Sakamoto, Phys. Lett B151 (1985) 115

[92] A. Salam and E. Sezgin (eds), Supergravities in Diverse Dimensions, vol. 1 and 2, (Word Scientific, Amsterdam, Singapore, 1989)

[93] G. Sardanashvily, Gauge Theory in Jet Manifolds (Hadronic Press, Palm Harbor, 1993); Five Lectures on the Jet Manifold. Methods in Field Theory E-print: hep-th/9411089

[94] D. J. Saunders, The Geometry of Jet Bundles (Cambridge Univ. Press, 1989)

[95] J. H. Schwarz (ed), Superstrings: the first 15 years of superstring theory: reprints $8 f$ commentary, (World Scientific, Singapore e. a., 1985)

[96] A. Sen, Phys. Rev. Lett. 55 (1986) 1846

[97] G. M. Shore, Nucl. Phys. B286 (1987) 349

[98] A. A. Tseytlin, Phys. Rev. D26 (1982) 3327

[99] A. A. Tseytlin, Phys. Lett. B178 (1986) 34

[100] A. A. Tseytlin, Nucl. Phys. B294 (1987) 383

[101] S. Vacaru, in "Foundations of Gravitation and Cosmology", Contributions to the International School-Seminar, Odessa, September 4-10, 1995 (Russian Gravitational Society, Moscow, 1995) p.131

[102] S. Vacaru, J. Math. Phys 37 (1996) 508

[103] S. Vacaru, Buletinul Academiei de Ştiinţe a Republicii Moldova, Fizica şi Tehnica 3 (1995) 53 
[104] S. Vacaru, Buletinul Academiei de Ştiinţe a Republicii Moldova, Fizica şi Tehnica 1 (1995) p. 54

[105] S. Vacaru, Buletinul Academiei de Ştiinţe a Republicii Moldova, Fizica şi Tehnica 1 (1996) p. 62

[106] S. Vacaru, Generalized Lagrange and Finsler Supergravity; E-print: grqc/9604016.

[107] S. Vacaru, Locally anisotropic gravity and strings, E-print: gr-qc/9604013.

[108] S. Vacaru, Locally anisotropic strings, (submitted to "Gravitation and Cosmology", 1996).

[109] S. Vacaru, Spinors in higher dimensional and locally anisotropic spaces; E-print: gr-qc/9604015.

[110] S. Vacaru, Locally anisotropic interactions: I. Nonlinear connections in higher order anisotropic superspaces; E-print: hep-th/9607194.

[111] S. Vacaru, Locally anisotropic interactions: II. Torsions and curvatures of higher order anisotropic superspaces; E-print: hep-th/9607195.

[112] S. Vacaru, Locally anisotropic interactions: III. Higher order anisotropic supergravity; E-print: hep-th/9607196.

[113] S. Vacaru and Yu. Goncharenko, Int. J. Theor. Phys. 34 (1995) 1955

[114] S. Vacaru and S. Ostaf, Buletinul Academiei de Ştiinţe a Republicii Moldova, Fizica şi Tehnica 3 (1993) 4

[115] S. Vacaru and S. Ostaf, in Coloquium on Differential Geometry, 25-30 July 1994 (Lajos Kossuth University, Debrecen, Hungary) p.56

[116] S. Vacaru and S. Ostaf, in Lagrange and Finsler Geometry, eds. P. L. Antonelli and R. Miron, ( Kluwer Academic Publishers, Dordrecht, Boston, London, 1996) 241

[117] S. Vacaru and S. Ostaf, Rep. Math. Phys. 37 (1996) 309; E-print: grqc/9602010

[118] S. Vacaru, S. Ostaf and Yu. Goncharenko, Romanian J. Physics 39 (1994) 199 
[119] S. Vacaru, S. Ostaf, Yu. Goncharenko and A. Doina, Buletinul Academiei de Ştiinţe a Republicii Moldova, Fizica şi Tehnica 3 (1994) 42

[120] V. S. Vladimirov and I. V. Volovich, Theor. Math. Phys. 59 (1984) 317 [in Russian]

[121] A. A. Vlasov, Statistical Distribution Functions (Nauka, Moscow, 1966) [in Russian]

[122] I. V. Volovich, Doklady Academii Nauk SSSR 269 (1975) 524 [in Russian]

[123] J. Wess and J. Bagger, Supersymmetry and Supergravity (Princeton University Press, 1983)

[124] J. Wess and B. Zumino, Phys. Lett. 37 (1971) 95

[125] P. West, Introduction to Supersymmetry and Supergravity (World Scientific, 1986)

[126] B. DeWitt, Supermanifolds (Cambridge University Press, 1984)

[127] E. Witten, Nucl. Phys. B266 (1986) 245

[128] K. Yano and S. I. Ishihara, Tangent and Cotangent Bundles. Differential Geometry (Marcel Dekker, New York, 1973)

[129] B. Zumino, Y. S. Wu and A. Zee, Nucl. Phys. 239 (1984) 